Aus der Klinik für Kardiologie und Pneumologie

(Prof. Dr. med. G. Hasenfuß)

im Zentrum Innere Medizin

der Medizinischen Fakultät der Universität Göttingen

\title{
Strukturiertes Training zur Tabakentwöhnung in einem Haus der Maximalversorgung: eine prospektive Studie
}

\author{
Inaugural - Dissertation \\ zur Erlangung des Doktorgrades \\ der Medizinischen Fakultät der \\ Georg-August-Universität zu Göttingen
}

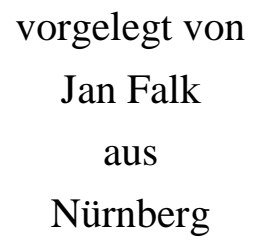


D e k a n:

I. Berichterstatter:

II. Berichterstatter:

III. Berichterstatter:

Tag der mündlichen Prüfung:
Prof. Dr. rer. nat. H. K. Kroemer

PD Dr. T. Raupach, MME

Prof. Dr. Wolfgang Himmel

14.10.2014 
Die Ergebnisse dieser Arbeit wurden teilweise veröffentlicht in:

Raupach T, Falk J, Vangeli E, Schiekirka S, Rustler C, Grassi MC, Pipe A, West R. (2012). Structured smoking cessation training for health professionals on cardiology wards: a prospective study. Eur J Prev Kardiol, im Druck 


\section{Inhaltsverzeichnis}

I. Inhaltsverzeichnis..........................................................................................

II. Abbildungsverzeichnis ................................................................................................................ VI

III. Tabellenverzeichnis...................................................................................................................VI

IV. Abkürzungen ................................................................................................................................. VII

1. Einleitung ............................................................................................................................................. 1

$1.1 \quad$ Epidemiologie des Rauchens .....................................................................

1.2 Rauchen und gesundheitliche Folgen ………………......................................

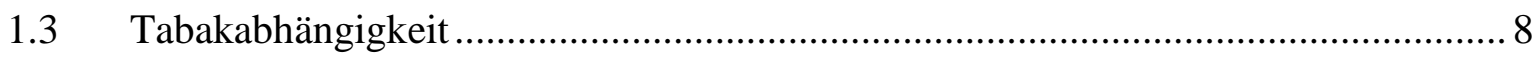

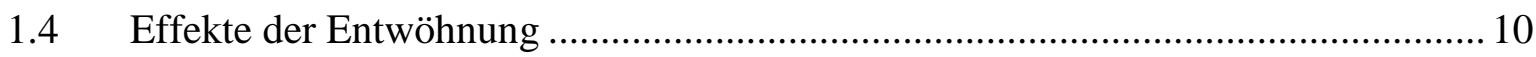

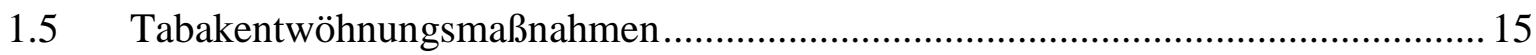

1.6 Empfehlungen zur Umsetzung der Tabakentwöhnungsbehandlung......................... 22

1.7 Entwöhnung bei hospitalisierten Patienten - aktuelle Versorgungslage ....................25

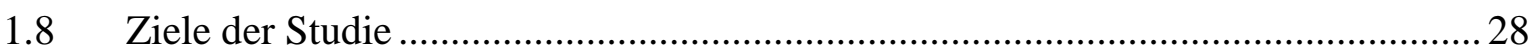

2. Material und Methoden ...........................................................................................................30

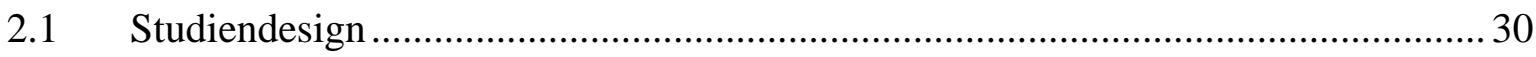

2.2 Probandenrekrutierung und Datensammlung.......................................................... 31

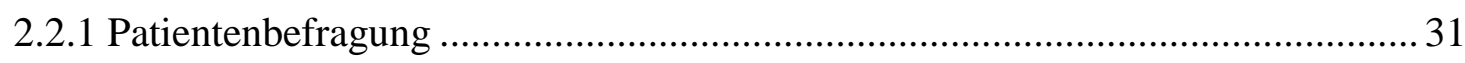

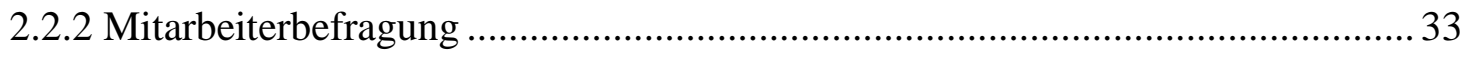

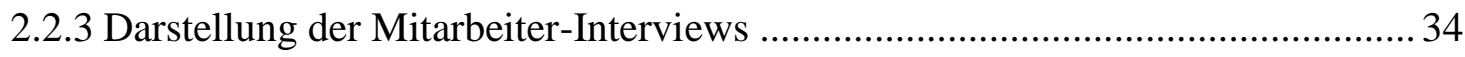

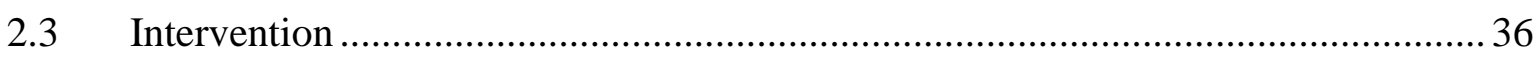

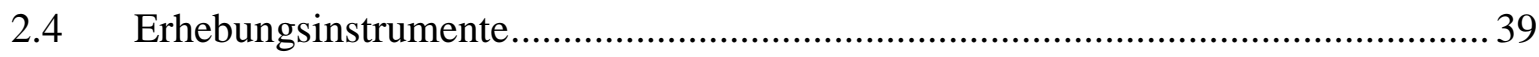

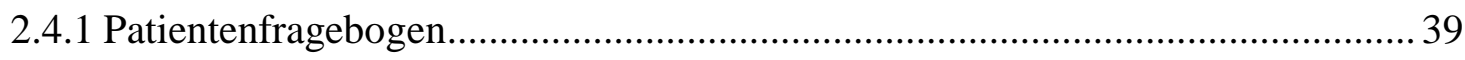

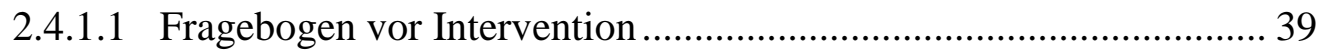

2.4.1.2 Fragebogen nach Intervention ....................................................... 40

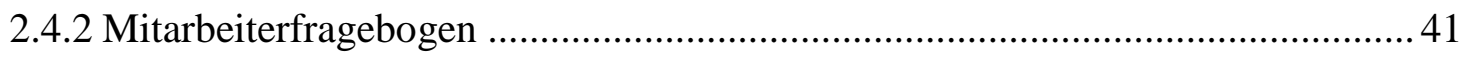

2.4.2.1 Erhebungsbogen Pflege vor Intervention......................................... 41

2.4.2.2 Erhebungsbogen Pflege nach Intervention ……………………...... 42

2.4.3 Leitfaden für die Mitarbeiter-Interviews ........................................................... 43 


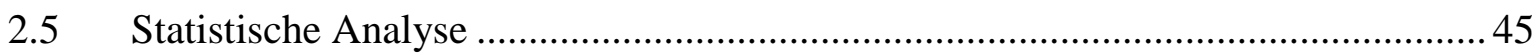

3. Ergebnisse ....................................................................................................................................... 47

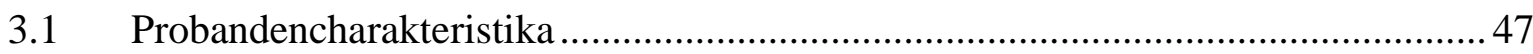

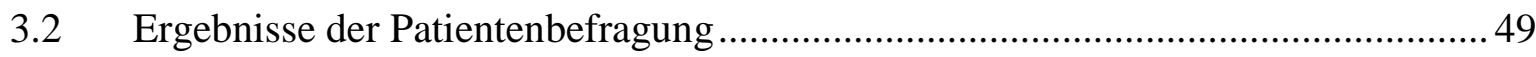

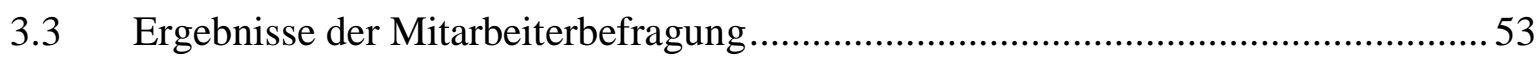

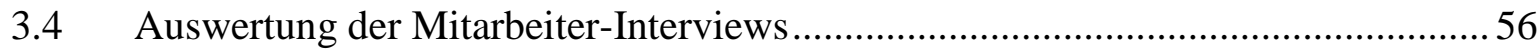

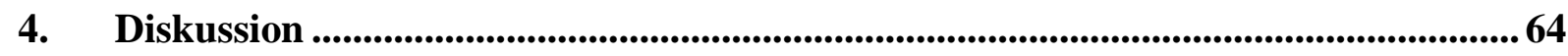

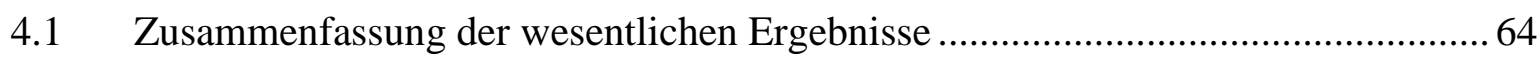

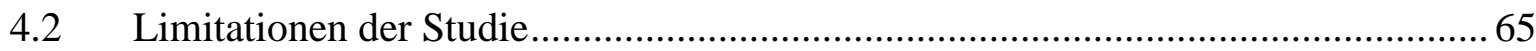

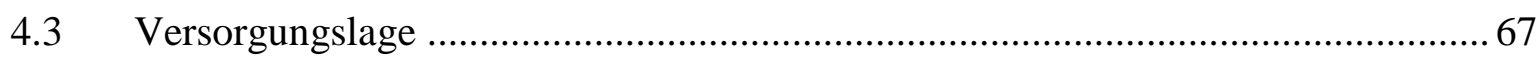

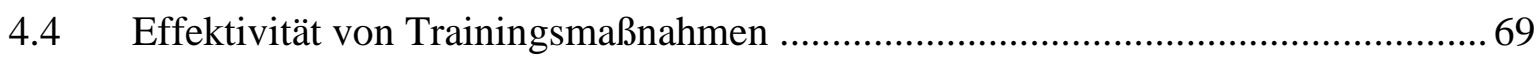

4.5 Systematische und theoretisch fundierte Herangehensweise mit Hilfe des Behaviour

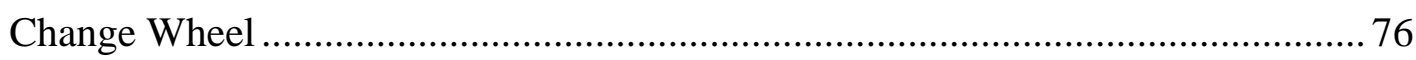

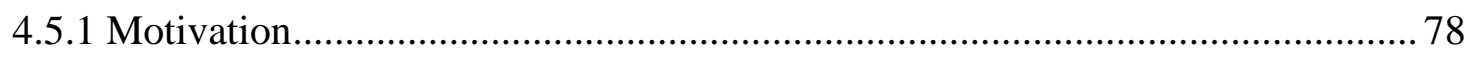

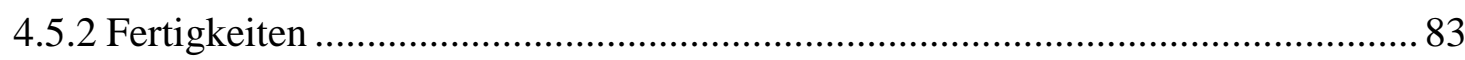

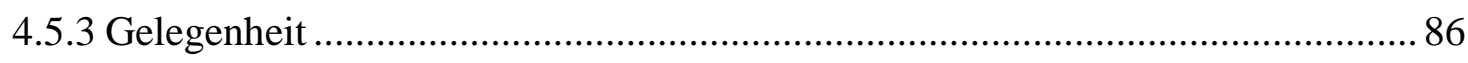

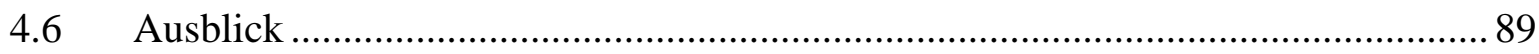

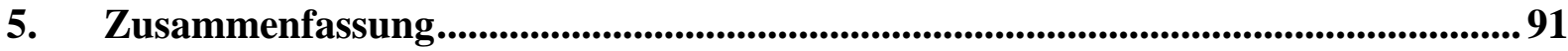

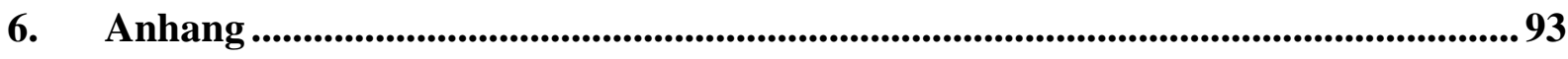

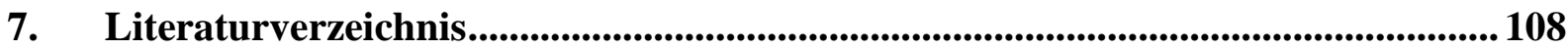




\section{Abbildungsverzeichnis}

Abb. 1: Aus „Rauchen - Aktuelle Entwicklungen bei Erwachsenen“ (Lampert 2011) ......... 1

Abb. 2: „COM-B“ - Modell, modifiziert nach Michie et al. 2011 ........................................ 27

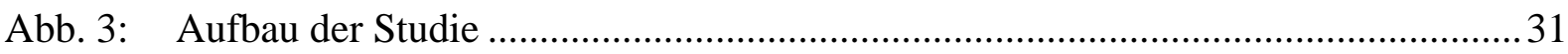

Abb. 4: : Behaviour Change Wheel (Michie et al. 2011) ....................................................... 76

\section{Tabellenverzeichnis}

Tab. 1: Metaanalyse: Wirksamkeit und Abstinenzraten verschiedener Medikamente sowie Medikamentenkombinationen verglichen mit Placebo 6 Monate nach dem Rauchstopp; modifiziert nach Fiore (Fiore et al. 2008) ....................................... 16

Tab. 2: $\quad$ 5-A-Schema; modifiziert nach Fiore (Fiore 2000) …............................................. 20

Tab. 3: Metaanalyse: Wirksamkeit und geschätzte Odds Ratio in Abhängigkeit von der Dauer der Beratung; modifiziert nach Fiore (Fiore 2000) .....................................20

Tab. 4: Patientencharakteristika, Rauchverhalten und Hauptaufnahmediagnosen angegeben in Prozent (absolute Zahlen) oder als Mittelwert \pm Standardabweichung 48

Tab. 5: Interventionshäufigkeit, Aufhörversuch mit eventueller Inanspruchnahme von Hilfe und Anbindung an die Tabakentwöhnungsambulanz angegeben in Prozent (absolute Zahlen)

Tab. 6: Wichtigster Grund für einen Entwöhnungsversuch im Klinikum. Angaben in Prozent (absolute Zahlen) 52

Tab. 7: Ergebnisse der Mitarbeiterbefragung, angegeben als prozentualer Anteil (absolute Zahlen) 54

Tab. 8: Darstellung der im Rahmen der Auswertung der transkribierten Interviews ermittelten Themen

Tab. 9: Verknüpfung der Elemente des „COM-B“-Modells mit den jeweils zur Verfügung stehenden Interventionsfunktionen; modifiziert nach Michie et al. 2011 


\section{Abkürzungen}

\begin{tabular}{|l|l|}
\hline Abb. & Abbildung \\
\hline ad lib. & ad libitum = nach Belieben \\
\hline BCW & Behaviour Change Wheel \\
\hline bzw. & beziehungsweise \\
\hline CO & Kohlenstoffmonoxid \\
\hline COPD & Chronisch obstruktive Lungenerkrankung \\
\hline CRP & C-reaktives Protein \\
\hline Dipl.- Päd. & Diplom-Pädagoge \\
\hline Dipl.- Psych. & Diplom-Psychologe \\
\hline DNRfK & $\begin{array}{l}\text { Deutsches Netz Rauchfreier Krankenhäuser und Gesundheitseinrich- } \\
\text { tungen }\end{array}$ \\
\hline engl. & Englisch \\
\hline EU & Europäische Union \\
\hline etc. & et cetera $=$ und so weiter \\
\hline FDA & Food and Drug Administration \\
\hline FEV 1 & forciertes exspiratorisches Volumen in der ersten Sekunde \\
\hline FTND & Fagerström-Test für Nikotinabhängigkeit \\
\hline GABA & $\gamma$-Aminobuttersäure \\
\hline HDL & High Density Lipoprotein \\
\hline IARC & International Agency for Research on Cancer \\
\hline IMC & Intermediate Care \\
\hline JCAHO & Joint Commission on Accreditation of Healthcare Organization \\
\hline KI & Konfidenzintervall \\
\hline KHK & koronare Herzerkrankung \\
\hline LDL & Low Density Lipoprotein \\
\hline$\mu$ g & Mikrogramm \\
\hline mg & Milligramm \\
\hline min & Minute \\
\hline ml & Milliliter \\
\hline $\mathrm{n}$ & Anzahl \\
\hline nAChR & nikotinerge Acetylcholinrezeptoren \\
\hline NSTEMI & Nicht-ST-Hebungsinfarkt \\
\hline NO & Stickstoffmonoxid \\
\hline NRT & nicotine replacement therapy = Nikotinersatztherapie \\
\hline OECD & Organisation für wirtschaftliche Zusammenarbeit und Entwicklung \\
\hline & \\
\hline
\end{tabular}




\begin{tabular}{|l|l|}
\hline OR & Odds Ratio \\
\hline PD & Privatdozent \\
\hline py & pack-years = Packungsjahre \\
\hline RKI & Robert Koch-Institut \\
\hline RR & Relatives Risiko \\
\hline S. & Seite \\
\hline s.o. & siehe oben \\
\hline SOP & Standard Operating Procedures \\
\hline SR & sustained release = Retardformulierung \\
\hline SPSS & Statistical Package for the Social Science (Statistikprogramm) \\
\hline SSRI & Selektive Serotonin -Wiederaufnahme - Hemmer \\
\hline STEMI & ST-Hebungsinfarkt \\
\hline Tab. & Tabelle \\
\hline t-PA & fibrinolytischer gewebsspezifischer Plasminogenaktivator \\
\hline UMG & Universitätsmedizin Göttingen \\
\hline UOHI & University of Ottawa Heart Institute \\
\hline WHO & World Health Organization \\
\hline Wo. & Wochen \\
\hline z.B. & zum Beispiel \\
\hline & \\
\hline
\end{tabular}

Aus Gründen der besseren Lesbarkeit beschränkt sich diese Arbeit auf die Verwendung männlicher Substantive (z.B. Arzt, Patient, Mitarbeiter, Raucher) bzw. Pronomina, bezieht sich damit jedoch grundsätzlich im gleichen Maße auch auf weibliche Personen (z.B. Ärztin, Patientin, Mitarbeiterin, Raucherin) - 


\section{1. $\quad$ Einleitung}

\subsection{Epidemiologie des Rauchens}

Laut einer Repräsentativerhebung des Robert Koch-Instituts (RKI), welche gleichermaßen die Daten für die Gesundheitsberichterstattung des Bundes liefert, raucht in der Bundesrepublik Deutschland fast ein Drittel der erwachsenen Bevölkerung. In absoluten Zahlen entspricht dies rund 20 Millionen Menschen (Lampert 2011).

Bei dieser Erhebung zur Ermittlung der gesundheitlichen Lage gaben 30\% der befragten 18jährigen und älteren Personen an, täglich (24\%) oder gelegentlich (6\%) Tabakwaren zu konsumieren (Lampert 2011). In der überwiegenden Zahl der Fälle wird dabei Zigarette geraucht. So favorisieren die Raucherinnen und Raucher die fabrikfertige Zigarette (83\% bzw. $76 \%$ ) vor selbstgedrehten Zigaretten (24\% bzw. $26 \%$ ), vor Zigarren/ Zigarillos (2\% bzw. $11 \%$ ) sowie der Pfeife $(0,2 \%$ bzw. $4 \%)$.

Bei der Betrachtung geschlechtsspezifischer Unterschiede liegen die Männer nach wie vor an der Spitze. Demnach beträgt die ermittelte Raucherquote $34 \%$ für Männer und $26 \%$ für Frauen (Lampert 2011).

Abb. 1: Aus „Rauchen - Aktuelle Entwicklungen bei Erwachsenen“ (Lampert 2011; Grafik links S.3, rechts S.5)
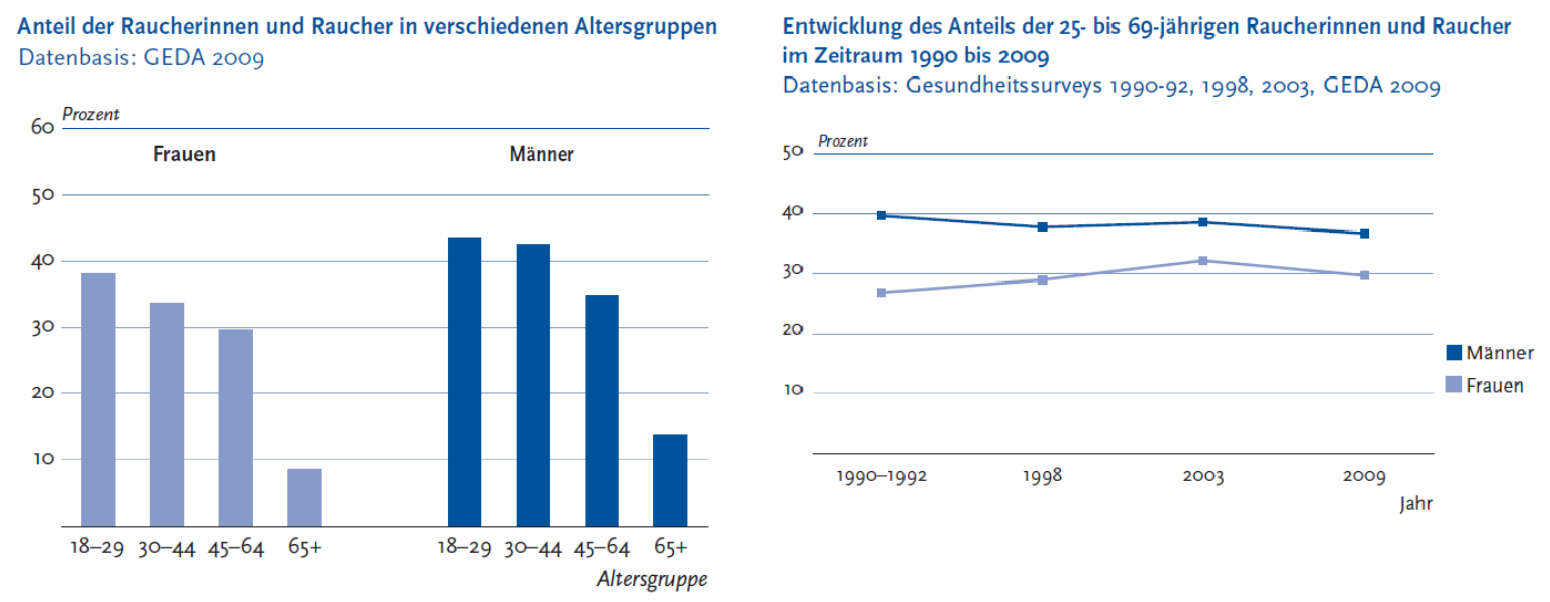

Differenziert man den Anteil der Raucherinnen und Raucher in verschiedene Altersstufen (siehe Abb. 1), so liegt die Raucherquote bei den 18- bis 29-Jährigen mit $38 \%$ aller befragten Frauen und $43 \%$ der befragten Männer jeweils am höchsten. Entsprechende Ergebnisse lieferten auch die Zahlen der Mikrozensus-Befragung des Statistischen Bundesamtes (Statistisches Bundesamt 2010). 
Hinsichtlich der Raucherprävalenz nimmt die Bundesrepublik Deutschland im europäischen Vergleich einen Platz im Mittelfeld ein. In einer von der Organisation für wirtschaftliche Zusammenarbeit und Entwicklung (OECD) initiierten aktuellen Studie zum Anteil der täglich rauchenden erwachsenen Personen liegt Deutschland mit 21,9 \% direkt unter dem EUDurchschnitt von $23 \%$ (OECD 2012).

Dabei zeigt der Trend in Deutschland (siehe Abb. 1) seit 2003 für beide Geschlechter einen leichten Rückgang der Raucherquote.

Tabakrauchen trägt nachweislich zur Genese zahlreicher und zum Teil schwerwiegender Erkrankungen bei, bei manchen sogar als wesentlicher Risikofaktor. In den Industrienationen gilt es weiterhin als Hauptursache für vermeidbare Sterbefälle (Perk et al. 2012). Allein in Deutschland sterben jedes Jahr etwa 110000 - 140000 Personen an einer tabakrauchassoziierten Krankheit (DKFZ 2002, John und Hanke 2001).

Volkswirtschaftlich betrachtet belaufen sich die durch das Rauchen verursachten jährlichen Gesundheitskosten laut WHO auf eine Milliarde US-Dollar in Australien, nahezu 7 Milliarden US-Dollar in Deutschland und 81 Milliarden US-Dollar in den Vereinigten Staaten (Guindon 2006). Eine andere Arbeit beziffert die in Deutschland durch das Rauchen entstehenden Gesamtkosten auf 21 Milliarden Euro, wovon etwa 7,5 Milliarden Euro auf die direkten medizinischen Kosten für die Behandlung rauchbedingter Erkrankungen entfallen (Neubauer et al. 2006).

\subsection{Rauchen und gesundheitliche Folgen}

Weltweit stirbt alle 6 Sekunden ein Mensch an einer Krankheit, die mit dem Rauchen assoziiert ist (Mathers und Loncar 2006, WHO 2008b). Schätzungen zufolge soll die tabakrauchassoziierte Mortalität von weltweit 5,4 Millionen Menschen in 2005 auf 6,4 Millionen in 2015 und 8,3 Millionen im Jahre 2030 ansteigen (Mathers und Loncar 2006).

Kaum ein Organsystem ist dabei von den Auswirkungen des Zigarettenrauchens ausgenommen (US DHHS 2004). Ursächlich sind die zahlreichen toxischen Stoffe, die der Raucher bei jedem Zigarettenzug inhaliert. Mittlerweile wurden über 4800 verschiedene Substanzen im Tabakrauch identifiziert (Hoffmann et al. 2001), wobei viele der Stoffe erst bei der Verbrennung des Tabaks entstehen (Roberts 1988). Unter ihnen befinden sich auch mehr als 50 Kar- 
zinogene, insbesondere Nitrosamine, aromatische Amine und polyzyklische aromatische Kohlenwasserstoffe (Hoffmann et al. 2001).

Es sind vor allem drei Krankheitsentitäten, die durch das Rauchen hervorgerufen werden. So entfallen annähernd ein Drittel aller tabakrauchassoziierten Sterbefälle auf Krebserkrankungen, $29 \%$ auf Herz-Kreislauf-Erkrankungen und ebenfalls etwa $29 \%$ auf pulmonale Erkrankungen (Mathers und Loncar 2006). Im Folgenden sollen insbesondere unter Berücksichtigung der genannten Erkrankungsgruppen die pathophysiologischen Auswirkungen des Rauchens dargestellt werden:

Um 1950 erschienen die ersten Publikationen, welche die bereits am Anfang des 20. Jahrhunderts geäußerten Vermutungen über einen möglichen epidemiologischen Zusammenhang zwischen dem Rauchen und dem Auftreten eines Bronchialkarzinoms erhärteten (Doll und Hill 1950, Mills und Porter 1950, Schrek et al. 1950, Wynder und Graham 1950). Mittlerweile ist der kausale Zusammenhang durch Erkenntnisse aus einer Vielzahl an Forschungsarbeiten belegt (IARC 2004).

Rauchen ist die Hauptursache für die Entstehung des Bronchialkarzinoms, welches nach wie vor den ersten Platz auf der Liste der häufigsten Krebssterbefälle weltweit einnimmt (Ferlay et al. 2008). Nach derzeitigen Erkenntnissen sind bei Frauen bis zu $60 \%$, bei Männern bis zu $90 \%$ der Lungenkrebserkrankungen auf das aktive Zigarettenrauchen zurückzuführen (IARC 2004, RKI 2010).

In einer europäischen Studie zum Risiko der Entwicklung eines Bronchialkarzinoms wird die Odds Ratio $(O R)$ bei männlichen Ex-Rauchern auf 7,5 (95\% - Konfidenzintervall [KI]: 6,2 9,1) und bei aktuellen männlichen Rauchern auf 23,9 (95 \% - KI: 19,7 - 29,0) beziffert (Simonato et al. 2001). Dabei steigt das Erkrankungsrisiko mit der Anzahl der durchschnittlich konsumierten Zigaretten pro Tag und den sogenannten Packungsjahren (pack-years, py) (Jockel et al. 1992, Mao et al. 2001, Osann et al. 2000, Risch et al. 1993, Rylander et al. 1996, Sankaranarayanan et al. 1994). Bei starken Rauchern beispielsweise, die einen durchschnittlichen Konsum von 20 - 29 Zigaretten pro Tag aufweisen und über 40 Jahre geraucht haben, liegt die OR für Männer bzw. für Frauen mit 44,8 (95 \% - KI: 35,73 - 56,32) bzw. mit 29,48 (95\% - KI: 18,05 - 48,14) entsprechend höher (Simonato et al. 2001).

Eine andere, im New England Journal of Medicine publizierte Studie ermittelte das Risiko für aktuelle Raucher im Vergleich zu lebenslangen Nichtrauchern (adjusted hazard ratio), an einem Bronchialkarzinom zu versterben: Dieses liegt für Frauen bei 17,8 (99 \% - KI: 11,4 27,8) und für Männer bei 14,6 (99 \% - KI: 9,1 - 23,4) (Jha et al. 2013). 
Die kanzerogene Wirkung des Rauchens beschränkt sich nicht nur auf die Lunge. 1985 konnte im Rahmen der Evaluation epidemiologischer Studien aus verschiedenen Ländern eine Assoziation mit dem Auftreten von Karzinomen der Mundhöhle, des Larynx, des Pharynx, des Ösophagus, des Pankreas, der Blase und des Nierenbeckens hergestellt werden (IARC 2004). Diese Liste der International Agency for Research on Cancer (IARC), einer Einrichtung der WHO, die sich mit der Zusammentragung und Aufarbeitung wissenschaftlicher Erkenntnisse hinsichtlich der Krebsursachenforschung befasst, wurde im Laufe der Jahre unter Hinzunahme weiterer aktueller Studien stetig erweitert. Sie umfasst ferner Karzinome von Nasennebenhöhlen, Nasopharynx, Magen, Leber, Niere, Zervix sowie die akute myeloische Leukämie, für die ebenfalls eine Korrelation mit dem Tabakrauchen aufgezeigt werden konnte (IARC 2004).

Eine äquivalente Zusammenstellung der Vielzahl an tabakrauchinduzierten Malignomen findet sich in dem Report des Surgeon General aus dem Jahre 2004 (US DHHS 2004).

Insgesamt ist das Rauchen heutzutage verantwortlich für ein Fünftel aller Krebssterbefälle in ganz Europa (Bray und Weiderpass 2009).

Im Hinblick auf das respiratorische System sind die Auswirkungen des Tabakrauchens keineswegs ausschließlich auf die Erhöhung des Bronchialkarzinomrisikos limitiert:

Die chronisch obstruktive Lungenerkrankung (chronic obstructive pulmonary diesease, $C O P D)$ ist weltweit eine der Hauptursachen für Morbidität und Mortalität. In der globalen Todesursachenstatistik der World Health Organization liegt die COPD mit insgesamt 3 Millionen Sterbefällen pro Jahr auf Platz 4 (WHO 2008a). Schätzungen zufolge soll sie bis zum Jahr 2020 auf Platz 3 vorrücken (Murray und Lopez 1997).

Rauchen stellt den Hauptrisikofaktor für die Entstehung dieser chronischen Atemwegserkrankung dar (Doll et al. 2004, Hudson und Mannino 2010, Shahab et al. 2006, Siafakas et al. 1995). So beträgt im Vergleich zu Nichtrauchern die OR für die Entwicklung einer klinisch signifikanten COPD (Stadium II-IV nach GOLD) für Raucher 6,3 (95 \% - KI: 4,2 - 9,5) (Lokke et al. 2006).

Eine Studie mit einer Follow-Up-Zeit von 25 Jahren konnte weiterhin zeigen, dass bei fortgesetztem Tabakkonsum über diesen Zeitraum etwa 30 - $40 \%$ der Raucher eine COPD entwickeln werden (Lokke et al. 2006). Dabei gibt es auch Hinweise auf geschlechtsspezifische Unterschiede. So sollen Frauen im Vergleich zu Männern bei gleicher Rauchexposition eine höhere Disposition zu einer entsprechenden Lungenschädigung aufweisen (Chapman 2004, Prescott et al. 1997). 
Maßgeblich für eine Verschlechterung der Lungenfunktion beziehungsweise für die Entwicklung einer COPD ist eine verstärkte jährliche Reduktion der $\mathrm{FEV}_{1}$ (forciertes exspiratorisches Volumen in der ersten Sekunde) von durchschnittlich $25 \mathrm{ml}$ bei Nichtrauchern auf $40 \mathrm{ml}$ bei Rauchern (Kerstjens et al. 1997). Die groß angelegte Lung Health Study liefert in diesem Zusammenhang noch höhere Werte für die jährliche $\mathrm{FEV}_{1}$-Abnahme bei kontinuierlichen Rauchern, welche bis zu $62 \pm 55 \mathrm{ml}$ pro Jahr beträgt (Scanlon et al. 2000). Außerdem konnte gezeigt werden, dass die erwähnten negativen Auswirkungen auf die pulmonale Funktion partiell reversibel sind. Die Lung Health Study wird daher in Kapitel 1.4 ausführlicher vorgestellt.

Neben der Entwicklung einer chronisch obstruktiven Lungenerkrankung weisen Raucher im Vergleich zu Nichtrauchern nicht nur eine erhöhte Mortalität an der COPD auf (Doll 1999), sondern auch generell mehr respiratorische Symptome und Abnormalitäten bezüglich der Lungenfunktion (Pauwels und Rabe 2004). Asthmapatienten mit begleitendem Tabakkonsum sind häufiger symptomatisch (Althuis et al. 1999) und zeigen zudem eine schlechtere medikamentöse Asthmakontrolle auf (Thomson und Spears 2005).

Insgesamt betrachtet ist das Risiko für Raucher, an einer respiratorischen Erkrankung zu versterben, dreimal so hoch wie für Nichtraucher, wobei es bei starken Rauchern ( > 25 Zigaretten pro Tag) nahezu fünfmal so hoch ist (Doll et al. 2004).

Nach Betrachtung der tabakrauchassoziierten Malignomerkrankungen sowie der respiratorischen Erkrankungen folgt nun eine Darstellung der Auswirkungen des Rauchens auf das kardiovaskuläre System.

Der in diesem Zusammenhang bisweilen bedeutendste zugrundeliegende Pathomechanismus ist die Entwicklung einer Arteriosklerose. Die endotheliale Dysfunktion gilt dabei als einer der frühesten Wegbereiter der arteriosklerotischen Veränderung (Barua et al. 2001, Brunner et al. 2005). Das intakte Endothel, welches als Antwort auf physiologische dilatierende Reize über eine Ausschüttung von Stickstoffmonoxid (NO) eine Vasodilatation herbeiführt sowie mit der Sekretion des fibrinolytischen gewebsspezifischen Plasminogenaktivators (t-PA) der Thrombenbildung entgegenwirkt, ist bei Rauchern in seiner Funktion entscheidend beeinträchtigt (endotheliale Dysfunktion) (Blann et al. 1998, Brunner et al. 2005, Celermajer et al. 1996, Zeiher et al. 1995). So konnte mittels intrakoronarer Dopplermessung gezeigt werden, dass das Rauchen unmittelbar zu einer Verengung der distalen sowie der proximalen Koronararterien führt, und das trotz eines gesteigerten Sauerstoffbedarfs des Herzmuskels (Quillen et al. 1993). 
Wesentlicher Faktor der endothelialen Dysfunktion ist das gestörte Gleichgewicht zwischen reaktiven Sauerstoffverbindungen und schützenden Antioxidantien, verursacht durch den erhöhten oxidativen Stress beim Tabakrauchen (Yanbaeva et al. 2007). Die beim Rauchen vermehrt anfallenden reaktiven Sauerstoffradikale, wie beispielsweise die Superoxide (Nedeljkovic et al. 2003), führen zum einen durch eine rasch ablaufende Reaktion mit dem endothelialen NO zu einer Verminderung der NO-Verfügbarkeit (Goldstein und Czapski 1995, Gryglewski et al. 1986). Zum anderen entstehen durch sie weitere oxidierende und zytotoxische Stoffe wie das Peroxynitrit, welches über zahlreiche pathologische Effekte im Endothel, unter anderem einer gesteigerten Lipidoxidation, ein wesentliches Element bei der Entstehung der arteriosklerotischen Veränderung darstellt (Kojda und Harrison 1999, Radi et al. 1991, White et al. 1994). Der Schaden am Endothel lässt sich dabei schon nach dem Rauchen einer einzigen Zigarette nachweisen (Karatzi et al. 2007).

Bereits subklinische Manifestation der Arteriosklerose wie eine Verdickung der beiden inneren Gefäßwandschichten der Karotisgefäße lassen sich in Ultraschalluntersuchungen darstellen, wobei eine positive Korrelation zwischen dem Ausprägungsgrad der arteriosklerotischen Wandverdickung und der Exposition gegenüber Zigarettenrauch besteht (Diez-Roux et al. 1995, Howard et al. 1994). Im Rahmen koronarangiografischer Untersuchungen konnte eine ähnliche Abhängigkeit der Schwere der arteriosklerotischen Affektion der Herzkranzgefäße und der Anzahl der gerauchten Zigaretten ermittelt werden (Herbert 1975, Ramsdale et al. 1985).

Verglichen mit Nichtrauchern finden sich bei Rauchern erhöhte Werte für Triglyzeride, Cholesterin und LDL-Cholesterin im Serum (Brischetto et al. 1983, Craig et al. 1989, Freeman et al. 1993). Die Serumkonzentration des schützenden, antiatherogenen HDL-Cholesterins ist dagegen vermindert (Garrison et al. 1978, Tuomilehto et al. 1986, Williams et al. 1979). Das Ausmaß dieser Fettstoffwechselstörung steht dabei in positiver Korrelation zu der Anzahl der gerauchten Zigaretten (Brischetto et al. 1983, Craig et al. 1989).

Als weiterer kardiovaskulärer Faktor wird die Gerinnung bzw. die Thrombogenität des Blutes beeinflusst. So kommt es bei Rauchern zu einer ebenfalls dosisabhängigen Erhöhung des Plasmafibrinogens (Sinha et al. 2005), zu einer gesteigerten Thrombozytenaggregation (Brunner et al. 2005, Krupski 1991, Reinders et al. 1986), zu einem Abfall des Protein CPlasmaspiegels (Conlan et al. 1993), zu einem Anstieg der Leukozyten (Blann et al. 1998) sowie zu erhöhten Werten des Faktors VIIa und des Plasminogenaktivatorinhibitors Typ 1 (Fisher et al. 2000), also zu einer insgesamt prothrombotischen Konstellation. Die Erhöhung 
des Plasmafibrinogens und die der weißen Blutzellen lassen sich sogar noch zehn Jahre nach der letzten Zigarette nachweisen (Yarnell et al. 1987).

Ebenso erhöht im Vergleich zu Nichtrauchern sind der Hämatokrit sowie die Blut- und Plasmaviskosität (Shimada et al. 2010, Wannamethee et al. 2005).

Neben den hämostatischen Veränderungen gibt es auch unmittelbare hämodynamische Konsequenzen, vor allem vermittelt über eine Stimulation des sympathoadrenergen Systems (Benowitz et al. 1982). So führt das Rauchen zu einer vermehrten Ausschüttung von Epinephrin und Norepinephrin (Cryer et al. 1976, Grassi et al. 1994) mit der Folge einer erhöhten Herzfrequenz, einer erhöhten Auswurfleistung, einem gesteigerten Blutdruck und einem daraus resultierenden erhöhten myokardialen Sauerstoffverbrauch (Ball und Turner 1974, Grassi et al. 1994, Trap-Jensen 1988, Winniford 1990, Zamir et al. 2006).

Wenngleich es sich bei den hier aufgeführten Mechanismen nicht um alle pathophysiologischen Auswirkungen des Zigarettenrauchens auf das Herz-Kreislauf-System handelt, so stellen sie dennoch die wesentlichen Faktoren für die Erhöhung des kardiovaskulären Risikos bei Rauchern dar.

In der INTERHEART-Studie wurde gezeigt, dass das Risiko für einen akuten Myokardinfarkt bei Rauchern im Vergleich zu Personen, die nie geraucht haben, um das Dreifache (OR: 2,95; 95 \% - KI: 2,77 - 3,14; p < 0,0001) erhöht ist (Teo et al. 2006). Im Rahmen dieser großen Studie, die insgesamt über 27000 Patienten aus 52 Ländern einbezog, konnte ferner ein annähernd linearer Zusammenhang zwischen der Anzahl der gerauchten Zigaretten und dem Infarktrisiko aufgezeigt werden. Dabei erhöhte sich das Infarktrisiko mit jeder zusätzlich gerauchten Zigarette jeweils um einen Absolutbetrag von weiteren 5,6\% (Teo et al. 2006).

Selbst bei einer geringen Konsummenge von 1 - 4 Zigaretten pro Tag lässt sich ein im Vergleich zu lebenslangen Nichtrauchern signifikant höheres Mortalitätsrisiko feststellen. So lag das Relative Risiko (RR) dieser „leichten Raucher“ bezogen auf die Gesamtsterblichkeit bei 1,47 für Frauen bzw. bei 1,57 für Männer. Differenziert nach einzelnen Erkrankungen ergab sich für die weiblichen Raucher beispielsweise ein RR von 2,94, an einer ischämischen Herzerkrankung zu versterben bzw. ein RR von 5,03, an einem Bronchialkarzinom zu versterben (Bjartveit und Tverdal 2005).

Nur 50\% der starken Raucher ( $\geq 25$ Zigaretten/Tag) erreichen das 70. Lebensjahr. Unter Nichtrauchern sind das im Vergleich dazu 80 \% (Doll et al. 1994). Die Hälfte aller regelmäßi- 
gen Zigarettenraucher, also jeder zweite, wird letztendlich an den Folgen des Tabakkonsums sterben, ein Viertel davon im mittleren Lebensalter (Doll et al. 2004, Doll et al. 1994).

\subsection{Tabakabhängigkeit}

Trotz der hinlänglich bekannten gesundheitsschädigenden Auswirkungen des Tabakrauchens gibt es in Deutschland etwa 20 Millionen aktive Raucher (Lampert 2011). In diesem Zusammenhang sollen die zugrundeliegenden Mechanismen der Tabakabhängigkeit dargestellt werden, gerade auch im Hinblick auf das Verständnis der zur Verfügung stehenden Entwöhnungsmaßnahmen.

Wesentlich für die Entstehung einer Abhängigkeit ist das in der Zigarette enthaltende Nikotin (Dani und De Biasi 2001, Stolerman und Jarvis 1995). Nach der Inhalation gelangen die Nikotinmoleküle in die Blutbahn und von dort, nach Überwindung der Blut-Hirn-Schranke, in das Gehirn. Die Gesamtzeit für diese Passage beträgt nur sieben Sekunden (Jiloha 2010, Russell 1980). Aufgrund ihrer agonistischen Eigenschaft binden die Nikotinmoleküle an nikotinerge Acetylcholinrezeptoren (nAChR). Diese Rezeptoren gibt es in verschiedenen Subtypen, die jeweils in ihren physiologischen und pharmakologischen Eigenschaften differieren und ganz unterschiedlich im Gehirn verteilt sind (Benowitz 2010, De Biasi und Salas 2008, Jiloha 2010, Mansvelder et al. 2003, Mansvelder und McGehee 2002). Der $\alpha_{4} \beta_{2}$-Rezeptor als ein Subtyp ist dabei einer der hauptsächlichen Vermittler der Abhängigkeit (Benowitz 2010, De Biasi und Salas 2008, Rollema et al. 2007).

Eine Aktivierung der nAChR durch Nikotin führt zu einer Ausschüttung verschiedener Neurotransmitter im Gehirn (Benowitz 2010, Jiloha 2010, Mansvelder und McGehee 2002). So wird unter anderem das dopaminerge System stimuliert, das sogenannte Belohnungssystem, welches über positive Empfindungen zur Verstärkung eines bestimmten Verhaltens führt und so auch eine essentielle Rolle bei der Entwicklung von Süchten spielt (Balfour 1994, Mansvelder et al. 2003, Nestler 2005). Der zugrundeliegende neurobiologische Mechanismus dieser Verhaltensverstärkung ist eine durch Nikotin induzierte Aktivierung der nAChRezeptoren von Neuronen im ventralen Tegmentum des Mittelhirns, deren Axone in den Nucleus accumbens projizieren und dort zu einer Erhöhung der Dopaminausschüttung führen (Balfour 1994, Jiloha 2010).

Umgekehrt konnte in anderen Arbeiten gezeigt werden, dass bei Vorliegen einer Läsion im Areal des ventralen Tegmentums oder einer medikamentös herbeigeführten Blockade der Do- 
paminfreisetzung im Nucl. accumbens eine deutliche Abschwächung des verstärkenden Effektes von Psychostimulantien wie Nikotin zu verzeichnen war (Corrigall et al. 1992, Louis und Clarke 1998, Lyness et al. 1979).

Entscheidend für den verstärkenden Effekt sind insbesondere auch das rasche Anfluten des Nikotins im Gehirn sowie die hohen Nikotinspiegel im Blut unmittelbar nach dem Rauchen einer Zigarette (Benowitz et al. 1988).

Essentiell für das Verständnis von Abhängigkeit und Toleranz ist zudem, dass es nicht nur zu einer Aktivierung, sondern auch zu einer nachfolgenden Deaktivierung der nAChR kommt. In Anwesenheit von Nikotin gehen so schon innerhalb von Sekunden bis Minuten die ersten Rezeptoren der dopaminergen Neurone in einen inaktiven Zustand über (Mansvelder et al. 2003, Pidoplichko et al. 1997, Wooltorton et al. 2003). Wie schnell dies geschieht, ist unter anderem abhängig von dem Rezeptorsubtyp sowie der vorherrschenden Nikotinkonzentration (Mansvelder et al. 2002, Wooltorton et al. 2003).

Mit abfallendem Nikotinspiegel gehen die Rezeptoren nach einer gewissen Zeit wieder in einen aktivierbaren Zustand über, d.h. sie sind wieder sensibel gegenüber Nikotin. Dies erklärt, warum das Verlangen nach einer Zigarette vor allem nach gewissen Abstinenzperioden wie beispielsweise nach dem Nachtschlaf auftritt, da die vorher deaktivierten $\alpha_{4} \beta_{2}$-Rezeptoren nun wieder aktivierbar sind (Dani und De Biasi 2001, Dani und Heinemann 1996). Langfristig führt diese Rezeptordesensibilisierung zu einer Neuroadaptation: Im Rahmen einer chronischen Nikotinzufuhr kommt es so zu einer Erhöhung der Anzahl der nAChR (Balfour 1994, Benowitz 2010, Benwell et al. 1988, Dani und De Biasi 2001, Jiloha 2010, Mansvelder und McGehee 2002). Diese Hochregulation betrifft vor allem die Rezeptorsubtypen mit einer hohen Affinität für Nikotin, besonders also die $\alpha_{4} \beta_{2}$-Rezeptoren auf den Nervenzellen im ventralen Tegmentum, deren zugehörige Axone in den Nucl. accumbens projizieren und so das dopaminerge System stimulieren (Buisson und Bertrand 2001).

Weiterhin nachweislich durch Nikotin beeinflusste Transmittersysteme im zerebralen Stoffwechsel und teilweise mitverantwortlich für die Entwicklung einer Abhängigkeit sind neben dem bereits dargestellten cholinergen System das serotoninerge, das adrenerge, das GABAerge und das glutamaterge System (Jiloha 2010, Mansvelder et al. 2002).

Die in diesem Kapitel dargestellten Wirkungen des Nikotins im Körper geben eine Übersicht über die wesentlichen physiologischen Mechanismen der Tabakabhängigkeit. Sie sind allerdings in ihren Einzelheiten viel diffiziler und komplexer und in ihrer Gesamtheit auch noch 
nicht vollständig verstanden. Dabei spielen neben den erläuterten molekularen Mechanismen noch weitere Faktoren eine Rolle bei der Tabakabhängigkeit, beispielsweise die Klassische und Operante Konditionierung, die Genetik, soziale Faktoren oder Einflüsse aus der Umwelt (Andreas et al. 2008, Batra et al. 2003, Jiloha 2010).

Es sind aber zum überwiegenden Teil die durch Nikotin hervorgerufene Beeinflussung der diversen Transmittersysteme sowie die ablaufenden neuroadaptatorischen Prozesse, die im Falle eines Abstinenzversuches die Entzugssymptome hervorrufen und das Verlangen nach einer Zigarette steigern (De Biasi und Salas 2008).

Welches Abhängigkeitspotential Nikotin hat, konnte in einer Untersuchung an Jugendlichen nachgewiesen werden. Es stellte sich heraus, dass initiale Symptome der Nikotinabhängigkeit schon nach dem Konsum weniger Zigaretten auftreten können, und zwar innerhalb von wenigen Tagen bis Wochen (DiFranza et al. 2000). Eine andere Befragung im Rahmen einer prospektiven Studie ergab, dass das Rauchen einer einzigen Zigarette im Alter von 11 Jahren dazu führt, dass diese Personengruppe in späteren Jahren eher zu einem regelmäßigen Tabakkonsum neigt als diejenigen Jugendlichen, die nie eine Zigarette probiert hatten (Fidler et al. 2006).

\subsection{Effekte der Entwöhnung}

Analog zu den oben aufgeführten gesundheitsschädigenden Auswirkungen des Tabakrauchens sollen nun die positiven Effekte einer Abstinenz dargestellt werden.

Schon wenige Tage bis Wochen nach einem Rauchstopp verbessern sich die physiologischen Parameter Herzfrequenz, Blutdruck und Compliance kleinerer Arterien (Oren et al. 2006), die Herzfrequenzvariabilität (Munjal et al. 2009) und die koronare Endothelfunktion (Hosokawa et al. 2008). Weiterhin optimieren sich die rheologischen Parameter Blutviskosität (Shimada et al. 2010) und Hämatokrit (Wannamethee et al. 2005) sowie andere kardiovaskuläre Risikogrößen wie beispielsweise das HDL/LDL Verhältnis (Eliasson et al. 2001, Erguder et al. 2006).

Die inflammatorischen Marker CRP, Fibrinogen und Leukozytenzahl fallen mit zunehmender Abstinenz ebenso ab, wenngleich sie auch noch bis zu 20 Jahre nach dem erfolgten Rauchstopp erhöht sind im Vergleich zu Personen, die nie geraucht haben (Wannamethee et al. 2005). Diese nach einem Rauchstopp zum Teil sehr schnell erfolgende Normalisierung bzw. 
Optimierung vieler Risikoparameter ist mit einer deutlichen Reduktion des kardiovaskulären Risikos und der Mortalität assoziiert.

In einer Studie an 209 hospitalisierten rauchenden Patienten mit akutem Koronarsyndrom führte eine dreimonatige Raucherentwöhnungsintervention bestehend aus einer Verhaltenstherapie mit kombinierter individueller Pharmakotherapie nicht nur zu einer Reduktion der ReHospitalisierungsrate (relative Risikoreduktion: $44 \%$ ). Auch die Gesamtsterblichkeit lag in der Entwöhnungsgruppe mit 2,8 \% niedriger als in der Kontrollgruppe mit $12 \%$, was eine relative Risikoreduktion von $77 \%$ bezogen auf die Mortalität bedeutete (Mohiuddin et al. 2007).

Welchen Stellenwert ein Rauchverzicht insbesondere für Patienten mit stattgehabtem akuten Myokardinfarkt in Bezug auf das Überleben einnimmt, wurde durch die Ergebnisse einer Metaanalyse unter Einschluss von über 5800 Patienten verdeutlicht. Die kombinierte OR für die Mortalität lag bei den fortan abstinenten Infarktpatienten bei 0,54 (95 \% - KI: 0,46 - 0,62) (Wilson et al. 2000). Im Vergleich dazu werden die Effekte der anderen, konventionellen Therapiemaßnahmen bei Patienten nach Myokardinfarkt mit einer OR von 0,75 (95 \% - KI: 0,71 - 0,79) für die thrombolytische Therapie, einer OR von 0,77 (95 \% - KI: 0,70-0,84) für Aspirin sowie einer OR von 0,88 (95\% - KI: 0,80-0,98) für Betablocker angegeben (Lau et al. 1992, Wilson et al. 2000).

Eine ähnliche, im Juli 2003 im Journal of the American Medical Association publizierte systematische Übersichtsarbeit bezüglich der Auswirkung eines Rauchstopps auf die allgemeine Mortalitätsrate bei KHK-Patienten (Kriterien: Vorangegangener Infarkt, stabile oder instabile Angina pectoris), bietet ein noch größeres Kollektiv: Es wurden insgesamt 665 Publikationen gescreent, von denen letztendlich 20 in die Studie einbezogen wurden. Die Gesamtzahl der hierin eingeschlossenen Raucher betrug 12603, bei einer mittleren follow-up-Zeit von 5 Jahren (2 - 26 Jahre). Das Ergebnis dieser Übersichtsarbeit zeigte eine 36\%-ige Reduktion des relativen Risikos bezüglich der Mortalität bei den KHK-Patienten, die sich fortan einem Rauchverzicht unterzogen hatten im Vergleich zu denjenigen Patienten, die weiterhin rauchten. Dieses Resultat war unabhängig von Faktoren wie Alter, Geschlecht, initialem kardialen Ereignis, Land der Studie sowie Jahr des Studienbeginns (Critchley und Capewell 2003).

Umgekehrt wiesen rückfällige Raucher nach stattgehabtem akuten Koronarsyndrom, die nach einer temporären Abstinenz im Rahmen der Hospitalisierung das Tabakrauchen wieder aufnahmen, eine dreimal so hohe Mortalität auf wie Patienten, die nach dem kardialen Ereignis abstinent blieben (Colivicchi et al. 2011). 
Laut der Deutschen Gesellschaft für Kardiologie stellt nicht zuletzt deshalb die Tabakentwöhnung vor allem bei Patienten mit koronarer Herzerkrankung eine der wichtigsten therapeutischen Einzelmaßnahmen dar (Dietz und Rauch 2003).

Ein konsequenter Rauchverzicht nach einem Herzinfarkt reduziert nachweislich das ReInfarktrisiko und soll nach drei Jahren Abstinenz demjenigen eines Nichtrauchers entsprechen (Rea et al. 2002). Auch andere Autoren kommen in ihren Arbeiten zu dem Schluss, dass das kardiovaskuläre Risiko nach mehreren Jahren der Abstinenz schließlich bis auf das Risiko eines lebenslangen Nichtrauchers sinkt (Dobson et al. 1991, Gordon et al. 1974, McGill 1988, Rosenberg et al. 1985, Rosenberg et al. 1990). In anderen Arbeiten hingegen wurde gezeigt, dass ein geringes Restrisiko bestehen bleibt (Cook et al. 1986, Negri et al. 1994).

Im Hinblick auf die einleitend dargestellten tabakrauchassoziierten respiratorischen Erkrankungen gibt es ebenfalls eine Vielzahl an Studien, die die positiven Auswirkungen eines Rauchverzichts herausstellen.

So sinkt mit zunehmender Abstinenz das Risiko der Entstehung eines Bronchialkarzinoms (Doll et al. 2004, Graham und Levin 1971, Hammond 1966, Wigle et al. 1980). In einer Arbeit wird das kumulative Risiko für aktuelle Raucher, bis zu einem Alter von 75 Jahren an einem Bronchialkarzinom zu versterben, mit 15,9 \% für Männer sowie mit 9,5\% für Frauen angegeben. Dieses Risiko reduziert sich bei männlichen Rauchern auf 9,9\%,6 \%, $3 \%$ und 1,7 \% im Falle eines Rauchstopps im Alter von 60, 50, 40 und 30 Jahren. Eine ähnliche Abnahme des Risikos in Abhängigkeit von der Abstinenzdauer ist auch bei den weiblichen Rauchern zu verzeichnen (Peto et al. 2000).

Nicht nur aus primärpräventiver Sicht (Siafakas et al. 1995), sondern auch bei manifester chronisch obstruktiver Lungenerkrankung (COPD) stellt die Raucherentwöhnung die mitunter entscheidende und effektivste Maßnahme zum Management der Erkrankung sowie der Progressionshemmung dar (Morgan und Britton 2003, Willemse et al. 2004).

In zahlreichen Arbeiten konnte der positive Effekt einer Tabakrauchabstinenz auf die Lungenfunktion bei Patienten mit chronischer Bronchitis oder COPD gezeigt werden, vornehmlich durch eine weniger starke jährliche Abnahme der $\mathrm{FEV}_{1}$ im Vergleich zu den weiterhin rauchenden Probanden (Barter und Campbell 1976, Comstock et al. 1970, Hughes et al. 1982, Peat et al. 1990, Postma et al. 1986, Scanlon et al. 2000). 
Eine diesbezüglich sehr bekannte und umfangreiche Studie ist die Lung Health Study. Eingeschlossen wurden Raucherinnen und Raucher im Alter von 35 bis 60 Jahren mit milder bis moderater bronchialer Obstruktion. Bei den Probanden, die erfolgreich mit dem Rauchen aufhörten, erhöhte sich die $\mathrm{FEV}_{1}$ im ersten Jahr um durchschnittlich $47 \mathrm{ml}$. In dem folgenden Zeitraum bis zum fünften Jahr nach dem Rauchstopp betrug die jährliche $\mathrm{FEV}_{1}$-Abnahme 31 $\pm 42 \mathrm{ml}$. Im Vergleich dazu nahm die $\mathrm{FEV}_{1}$ bei den kontinuierlichen Rauchern im ersten Jahr durchschnittlich um $49 \mathrm{ml}$ ab, während sie dann weiter bis zum fünften Jahr um $62 \pm 55 \mathrm{ml}$ pro Jahr abnahm, also etwa doppelt so schnell im Vergleich zu den abstinenten Probanden (Scanlon et al. 2000).

Dieser Effekt zeigte sich auch noch über die fünfjährige Follow-up-Phase hinaus als stabil: 11 Jahre nach Beginn der Lung Health Study konnten 77,4 \% der ursprünglich 5887 Studienteilnehmer für eine erneute spirometrische Messung rekrutiert werden. Die jährliche $\mathrm{FEV}_{1^{-}}$ Abnahme war entsprechend niedriger bei den Männern und Frauen, die seit Beginn der Studie abstinent waren $(30,2 \mathrm{ml} / \mathrm{Jahr}$ sowie $21,5 \mathrm{ml} / \mathrm{Jahr})$ verglichen mit denjenigen Männern und Frauen, die über diesen Zeitraum weiterhin geraucht hatten $(66,1 \mathrm{ml} / \mathrm{Jahr}$ sowie $54,2 \mathrm{ml} / \mathrm{Jahr})$ (Anthonisen et al. 2002).

Das im Rahmen der Lung Health Study durchgeführte 10-wöchige Raucherentwöhnungsprogramm, bestehend aus einem eingehenden ärztlichen Ratschlag zum Rauchverzicht, 12 verhaltenstherapeutischen Gruppensitzungen und dem Einsatz von Nikotinkaugummis schlug sich auch in der Gesamtmortalität nieder. Die Gesamtmortalität wurde nach 14,5 Jahren ermittelt und demonstrierte eine signifikant höhere Sterberate in der usual care-Gruppe (10,38 pro 1000 Personenjahre versus 8,83 pro 1000 Personenjahre; $\mathrm{p}=0,03$ ) - obwohl selbst in der Interventionsgruppe nur knapp $20 \%$ der Studienteilnehmer eine kontinuierliche Abstinenz erreicht hatten (Anthonisen et al. 2005). Die Intervention wirkte sich somit auf die Mortalität der Gesamtgruppe aus, obwohl sie nur bei jedem fünften Studienteilnehmer den gewünschten Effekt (d.h. eine dauerhafte Entwöhnung) hatte.

Ein Rauchverzicht hat einen maßgeblichen Einfluss auf die Lebenserwartung, und zwar unabhängig davon, in welchem Alter dieser vorgenommen wird (Doll et al. 2004, Jha et al. 2013). Bei einem Rauchstopp im Alter von 60 Jahren gewinnt eine fortan abstinente Person im Durchschnitt drei Lebensjahre. Dabei ist der Effekt umso größer, je früher die Tabakabstinenz 
beginnt. So führt ein Tabakverzicht im Alter von 50, 40 oder 30 Jahren zu einer durchschnittlichen Erhöhung der Lebenserwartung um 6, 9 bzw. 10 Lebensjahre (Doll et al. 2004).

Das Pendant zur letztgenannten Studie, welche ausschließlich Männer einschloss, stellt eine Erhebung an über einer Million Frauen dar. Auch hier zeigte sich, dass mit zunehmender Abstinenzdauer das Relative Risiko bezogen auf die Mortalität stetig abnimmt. Bei Frauen mit einem Rauchstopp im Alter zwischen 45-54 Jahren beträgt das RR 1,56 (95 \% - KI: 1,49 1,64), im Alter von 35 - 44 Jahren 1,20 (95 \% - KI: 1,14 - 1,26) und im Alter von 25 - 34 Jahren 1,05 (95 \% - KI: 1,00 - 1,11) (Pirie et al. 2013). 


\subsection{Tabakentwöhnungsmaßnahmen}

Obwohl sich viele Raucher der schädigenden Wirkung bewusst sind und durchaus gewillt sind, aufzuhören, schaffen es im Endeffekt nur die wenigsten, dauerhaft abstinent zu bleiben (Benowitz 2010, Hughes et al. 1992, Hughes et al. 2004).

Im Rahmen einer Repräsentativerhebung im Auftrag des Bundesministeriums für Gesundheit sagten lediglich 19,4 \% der befragten Raucherinnen und Raucher, dass sie gegenwärtig nicht vorhaben, mit dem Tabakrauchen aufzuhören, während die Mehrzahl eine Aufhörbereitschaft zeigte oder diese gerade umgesetzt hatte (Kraus und Augustin 2001). Unter den Rauchern, die sich für einen Abstinenzversuch entschieden haben, nahmen 74,6 \% keine Hilfsmittel in Anspruch (Kraus und Augustin 2001).

Gerade bei dieser Personengruppe ist die Rückfallquote besonders hoch. Eine Analyse mehrerer Studien konnte zeigen, dass die Langzeitabstinenzrate bei den Rauchern, die ihren Aufhörversuch gänzlich ohne unterstützende Maßnahmen durchführten, bei nur 3 - $5 \%$ lag. Die Mehrzahl der Rückfälle ereignete sich dabei bereits in den ersten 8 Tagen nach dem Rauchstopp (Hughes et al. 2004). Auch andere Arbeiten konnten ähnlich niedrige Langzeitabstinenzraten bei diesen sogenannten ,unassisted quitters“ ermitteln (Baillie et al. 1995).

Mit alleiniger Willenskraft ist es offensichtlich schwierig, die Tabakabhängigkeit zu überwinden.

Um entwöhnungswillige Raucher bei ihrem schwierigen und oft von Rückfällen gekennzeichneten Aufhörversuch zu unterstützen, existieren eine ganze Reihe verschiedener Hilfsmittel und Entwöhnungsmaßnahmen. Prinzipiell unterscheidet man dabei zwischen medikamentösen und verhaltenstherapeutischen Ansätzen. Viele der zur Verfügung stehenden Maßnahmen wurden im Zuge der evidenzbasierten Medizin in zahlreichen Studien und Metaanalysen untersucht und schließlich auch in Form von Leitlinien hinsichtlich Effektivität, wissenschaftlicher Fundierung, Praktikabilität und Wirtschaftlichkeit evaluiert. Eine solche wichtige Zusammenstellung dieser über Jahre gewonnenen Erkenntnisse sind die Clinical Practice Guidelines, die im Jahre 2008 aktualisiert wurden (Fiore et al. 2008). An ihnen orientieren sich auch die deutschen Leitlinien (Andreas et al. 2008, Schmidt et al. 2006).

Im Folgenden werden die wichtigsten Entwöhnungsmaßnahmen kurz vorgestellt, beginnend mit einer übersichtsweisen Darstellung (siehe Tab. 1) der zum Einsatz kommenden Medikamente und ihren jeweiligen Wirksamkeitszahlen: 
Tab. 1: Metaanalyse: Wirksamkeit und Abstinenzraten verschiedener Medikamente sowie Medikamentenkombinationen verglichen mit Placebo 6 Monate nach dem Rauchstopp; modifiziert nach Fiore (Fiore et al. 2008)

\begin{tabular}{|c|c|c|c|}
\hline Medikation & $\begin{array}{l}\text { Stu- } \\
\text { dien- } \\
\text { arme }\end{array}$ & $\begin{array}{l}\text { Geschätzte Odds } \\
\text { Ratio (95 \% - KI) }\end{array}$ & $\begin{array}{l}\text { Geschätzte Absti- } \\
\text { nenzrate (95 \% - KI) }\end{array}$ \\
\hline Placebo & 80 & 1,0 & 13,8 \\
\hline \multicolumn{4}{|l|}{ Monotherapien } \\
\hline Vareniclin (2 mg/Tag) & 5 & $3,1(2,5-3,8)$ & $33,2(28,9-37,8)$ \\
\hline Nikotin Nasenspray & 4 & $2,3(1,7-3,0)$ & $26,7(21,5-32,7)$ \\
\hline $\begin{array}{l}\text { Hochdosiertes Nikotinpflaster } \\
\text { ( > } 25 \mathrm{mg} \text { ) (Umfassen sowohl } \\
\text { Standard als auch Langzeit- } \\
\text { Dauer) }\end{array}$ & 4 & $2,3(1,7-3,0)$ & $26,5(21,3-32,5)$ \\
\hline $\begin{array}{l}\text { Langzeit Nikotinkaugummi } \\
(>14 \text { Wo.) }\end{array}$ & 6 & $2,2(1,5-3,2)$ & $26,1(19,7-33,6)$ \\
\hline Vareniclin $(1 \mathrm{mg} / \mathrm{Tag})$ & 3 & $2,1(1,5-3,0)$ & $25,4(19,6-32,2)$ \\
\hline Nikotin Inhaler & 6 & $2,1(1,5-2,9)$ & $24,8(19,1-31,6)$ \\
\hline Clonidin & 3 & $2,1(1,2-3,7)$ & $25,0(15,7-37,3)$ \\
\hline Bupropion SR & 26 & $2,0(1,8-2,2)$ & $24,2(22,2-26,4)$ \\
\hline Nikotinpflaster (6 - 14 Wo.) & 32 & $1,9(1,7-2,2)$ & $23,4(21,3-25,8)$ \\
\hline Langzeit Nikotinpflaster & 10 & $1,9(1,7-2,3)$ & $23,7(21,0-26,6)$ \\
\hline Nortriptylin & 5 & $1,8(1,3-2,6)$ & $22,5(16,8-29,4)$ \\
\hline Nikotinkaugummi (6 - 14 Wo.) & 15 & $1,5(1,2-1,7)$ & $19,0(16,5-21,9)$ \\
\hline \multicolumn{4}{|l|}{ Kombinationstherapien } \\
\hline $\begin{array}{l}\text { Pflaster (Langzeit; > } 14 \text { Wo.) + } \\
\text { ad lib. NRT (Kaugummi oder } \\
\text { Spray) }\end{array}$ & 3 & $3,6(2,5-5,2)$ & $36,5(28,6-45,3)$ \\
\hline Pflaster + Bupropion SR & 3 & $2,5(1,9-3,4)$ & $28,9(23,5-35,1)$ \\
\hline Pflaster + Nortriptylin & 2 & $2,3(1,3-4,2)$ & $27,3(17,2-40,4)$ \\
\hline Pflaster + Inhaler & 2 & $2,2(1,3-3,6)$ & $25,8(17,4-36,5)$ \\
\hline $\begin{array}{l}\text { Pflaster }+2 . \text { Generations- } \\
\text { Antidepressiva (Paroxetin, } \\
\text { Venlafaxin) }\end{array}$ & 3 & $2,0(1,2-3,4)$ & $24,3(16,1-35,0)$ \\
\hline \multicolumn{4}{|c|}{ Medikamente, die sich als nicht effektiv erwiesen } \\
\hline $\begin{array}{l}\text { Selektive Serotonin-Wieder- } \\
\text { aufnahme - Hemmer (SSRIs) }\end{array}$ & 3 & $1,0(0,7-1,4)$ & $13,7(10,2-18,0)$ \\
\hline Naltrexon & 2 & $0,5(0,2-1,2)$ & $7,3(3,1-16,2)$ \\
\hline
\end{tabular}




\section{Nikotinersatztherapie (NRT)}

Nikotinersatzpräparate führen dem Körper Nikotin in niedrigerer Dosierung zu, ohne ihn dabei den im Tabakrauch enthaltenen schädlichen Toxinen und Kanzerogenen auszusetzen (Henningfield und Keenan 1993, Sees 1990).

Außerdem wird das Nikotin hier im Vergleich zum Rauchen deutlich langsamer aufgenommen. So liegt beim Rauchen der Maximalwert der Nikotinaufnahme nach fünf Minuten bei $210 \mu \mathrm{g} / \mathrm{min}$, während er beim Nikotinkaugummi erst nach zehn Minuten mit $64 \mu \mathrm{g} / \mathrm{min}$ sein Maximum erreicht (Benowitz et al. 1988). Der für die Entwicklung einer Abhängigkeit wesentliche Effekt der Verstärkung ausgelöst durch das schnelle Anfluten im Gehirn sowie die hohen Nikotinplasmaspiegel, wie sie nach dem Rauchen einer Zigarette auftreten (siehe auch Kapitel 1.3), wird, bei korrekter Applikation, durch die Kinetik der Nikotinersatztherapie nicht erzielt. Nikotinersatzmittel rufen demnach bei Nichtrauchern keine Abhängigkeit hervor (Hughes 1988).

Ziel der Nikotinersatztherapie ist es, die oft für die Rückfälle verantwortliche Entzugssymptomatik sowie das Rauchverlangen zu mindern, welche durch den abfallenden Nikotinspiegel und die nun wieder aktivierbaren nACh-Rezeptoren zustande kommen (Balfour und $\mathrm{Fa}$ gerstrom 1996, Fiore et al. 2008, Henningfield 1995).

Die Präparate gibt es in den Darreichungsformen Kaugummi, Pflaster, Nasalspray, Lutschtablette, Inhaler und Sublingualtablette, die sich jeweils in ihrer Kinetik unterscheiden. Hinsichtlich der Wirksamkeit bezifferte die Cochrane Tobacco Addiction Group unter Hinzuziehung von 123 Studien unabhängig von der Applikationsform die OR bezogen auf die Langzeitabstinenz auf 1,77 (95 \% - KI: 1,66 - 1,88) (Silagy et al. 2004). Das Spektrum reichte dabei von einer OR von 1,66 (95 \% - KI: 1,52 - 1,81) für das Kaugummi bis hin zur effektivsten Darreichungsform als Nasalspray mit einer OR von 2,35 (95 \% - KI: 1,63 - 3,38).

Die in den Clinical Practice Guidelines aufgeführten Langzeitabstinenzraten unter Zuhilfenahme nikotinhaltiger Präparate liegen zwischen 19 und 26,7 \%. Diese lässt sich weiterhin durch eine Kombination bestimmter Präparate steigern. So wird die Langzeitabstinenzrate bei einem kombinierten Einsatz eines Nikotinpflasters mit einem Spray oder einem Kaugummi auf 36,5\% beziffert (Fiore et al. 2008).

Die unerwünschten Nebenwirkungen der oral angewandten Nikotinersatzpräparate sind lokale Irritationen der Mund- und Rachenschleimhaut, ein scharfer, brennender Geschmack sowie gastrointestinale Symptome und Sodbrennen (Balfour und Fagerstrom 1996). Insgesamt sind diese Nebenwirkungen eher milde ausgeprägt mit teilweise erfolgender Abmilderung im Lau- 
fe der Therapie (Jarvis et al. 1982, Tonnesen et al. 1988). Bei Applikation des Nikotinpflasters können als Nebenwirkungen lokale Hautreaktionen auftreten (Henningfield 1995) sowie dosisabhängig Schlafstörungen (Transdermal Nicotine Study Group 1991).

Das kardiovaskuläre Risiko hingegen wird durch die Inanspruchnahme der Nikotinersatztherapie nicht erhöht (Benowitz und Gourlay 1997, Frishman et al. 2001, Haustein et al. 2002). In zahlreichen Studien konnte selbst bei Patienten mit einer koronaren Herzerkrankung oder gar bei Vorliegen eines akuten Koronarsyndroms keine Zunahme des Risikos festgestellt werden (Joseph et al. 1996, Tzivoni et al. 1998, Woolf et al. 2012). In einer Studie an 187 aufgrund eines akuten Koronarsyndroms hospitalisierten Patienten zeigte der unmittelbare Vergleich von Patienten ohne Nikotinpflaster mit Patienten, die ein solches Pflaster erhielten, keinen Unterschied hinsichtlich der Mortalität. Diese betrug nach 7 Tagen (kein Nikotinpflaster vs. Nikotinpflaster, absolute Zahlen in Klammern) $0,5 \%$ (1) vs. $0 \%$ (0), nach 30 Tagen $1,6 \%$ (3) vs. $1,1 \%$ (2) und nach einem Jahr 5,4 \% (10) vs. 4,8 \% (9) (Meine et al. 2005).

\section{Bupropion}

Das Antidepressivum Bupropion wurde 1997 in den USA von der Food and Drug Administration (FDA) als erstes nikotinfreies Präparat zur Tabakentwöhnung zugelassen. Der exakte Wirkmechanismus ist bis heute unklar. Eine wesentliche Rolle scheint neben der Beeinflussung des zerebralen noradrenergen und dopaminergen Systems (Ascher et al. 1995) auch ein Antagonismus an den nikotinergen Acetylcholinrezeptoren (nAChR) zu spielen (Fryer und Lukas 1999, Mansvelder et al. 2007). Nach einer Behandlung mit Bupropion sollen so zwischen 75 - $95 \%$ der nAChR dopaminerger Neurone im ventralen Tegmentum in ihrer Aktivität inhibiert sein (Mansvelder et al. 2007).

Die Arbeit der Cochrane Collaboration zur Einschätzung der Effektivität antidepressiver Medikamente bei der Tabakentwöhnung, welche 36 Studien mit insgesamt 11140 Probanden und Bupropion als alleinigem Entwöhnungsmittel zusammenfasste, konnte einen signifikanten Anstieg der Langzeitabstinenzrate (RR: 1,69; $95 \%$ - KI: 1,53 - 1,85) zeigen (Hughes et al. 2007). Dabei lag die durchschnittliche Aufhörquote nach mindestens 6 Monaten follow-upZeit bei $17 \%(4 \%-43 \%)$ in der Bupropion-Gruppe und bei $9 \%(0 \%$ - $22 \%)$ in der Kontrollgruppe. Ähnliche Ergebnisse liefern die Clinical Practice Guidelines, ihrerseits unter Einschluss von 24 Studien (Fiore et al. 2008). 
Die am häufigsten auftretenden Nebenwirkungen unter Bupropion sind Schlaflosigkeit, Mundtrockenheit, Schwindel und Kopfschmerzen (Fossati et al. 2007, Jorenby et al. 1999, Tonnesen et al. 2003). Als schwerwiegende Komplikation wurde das Auftreten von zerebralen Krampfanfällen bei höherer Dosierung mit einer ungefähren Häufigkeit von 1:1000 beschrieben (Dunner et al. 1998). Infolgedessen besteht eine Kontraindikation für Patienten mit bestehendem Anfallsleiden (Batra 2001, Fiore et al. 2008).

\section{Vareniclin}

Das Anfang 2007 in Deutschland zugelassene Medikament Vareniclin weist im Vergleich zu den Nikotinersatzpräparaten am nAChR agonistisch-antagonistische Eigenschaften auf: In Abwesenheit von Nikotin wirkt es einerseits als Partialagonist an den $\alpha_{4} \beta_{2}$-Rezeptoren und reduziert so, genau wie die NRT, die Entzugssymptomatik. Bei Anwesenheit von Nikotin hingegen, also beim Rückfall, wirkt Vareniclin antagonistisch an diesen Rezeptoren und mindert so die Genusswirkung des Rauchens (Rollema et al. 2007).

Studien zeigen eine Überlegenheit gegenüber Bupropion sowie eine geringfügig höhere Effektivität im Vergleich zur NRT (Aubin et al. 2008, Cahill et al. 2011). In einer weiteren Vergleichsstudie lag die Langzeitabstinenzrate nach 52 Wochen bei $23 \%$ in der VareniclinGruppe verglichen mit 10,3 \% in der Placebo-Gruppe (OR: 2,66; $95 \%$ - KI: 1,72 - 4,11; p < 0,001) und 14,6\% in der Bupropion-Gruppe (OR: 1,77; $95 \%$ - KI: 1,19 - 2,63; p = 0,004) (Jorenby et al. 2006).

Die am häufigsten angegebene Nebenwirkung unter Vareniclin ist Übelkeit. Je nach Studie tritt sie bei der empfohlenen Gabe von $1 \mathrm{mg}$ Vareniclin zweimal täglich bei 28,1\% bis 40,2 \% der Patienten auf, sie ist dosisabhängig und von eher milder bis moderater Intensität (Aubin et al. 2008, Ebbert et al. 2010, Gonzales et al. 2006, Rennard et al. 2012, Williams et al. 2007). Weitere Nebenwirkungen sind Kopfschmerzen und Schlafstörungen (Aubin et al. 2008, Bolliger et al. 2011). Bedenken, Vareniclin führe zu einer erhöhten Suizidalität, suizidalen Gedanken oder Depressionen, konnten nicht bestätigt werden (Gunnell et al. 2009).

Eine aktuelle und umfassende Metaanalyse aller bisher publizierten, randomisierten Kontrollstudien zum Einsatz von Vareniclin im Rahmen der Tabakentwöhnung zeigte keine signifikante Erhöhung des kardiovaskulären Risikos (Prochaska und Hilton 2012). 


\section{Kurzintervention}

Eine kurze Beratung kann nach dem 5-A-Schema (ask, advise, asses, assist, arrange; siehe auch Tab. 2) oder bei weniger motivierten Rauchern nach dem 5-R-Schema (relevance, risks, rewards, roadblocks, repetition) erfolgen (Anderson et al. 2002).

Tab. 2: 5-A-Schema; modifiziert nach Fiore (Fiore 2000)

\begin{tabular}{|l|l|}
\hline Ask about tobacco use & $\begin{array}{l}\text { Identifikation und Dokumentation des Rauchstatus } \\
\text { eines jeden Patienten während des Arztkontaktes }\end{array}$ \\
\hline Advice to quit & $\begin{array}{l}\text { Klarer, eindeutiger und patientenbezogener Ratschlag } \\
\text { zum Rauchverzicht }\end{array}$ \\
\hline $\begin{array}{l}\text { Assess willingness to make a quit } \\
\text { attempt }\end{array}$ & $\begin{array}{l}\text { Ist der Raucher zum jetzigen Zeitpunkt bereit, einen } \\
\text { Rauchverzicht zu unternehmen? }\end{array}$ \\
\hline Assist in quit attempt & $\begin{array}{l}\text { Der entwöhnungswillige Patient soll mit Beratung } \\
\text { und medikamentöser Therapie unterstützt werden }\end{array}$ \\
\hline Arrange follow-up & $\begin{array}{l}\text { Vereinbarung einer Wiedervorstellung des Patienten, } \\
\text { vorzugsweise in der ersten Woche nach Beginn der } \\
\text { Abstinenz }\end{array}$ \\
\hline
\end{tabular}

Dabei korreliert die Langzeitabstinenz mit der Intensität der Beratung, dargestellt in der abgebildeten tabellarischen Übersicht aus den Clinical Practice Guidelines (siehe Tab. 3) (Fiore 2000).

Tab. 3: Metaanalyse: Wirksamkeit und geschätzte Odds Ratio in Abhängigkeit von der Dauer der Beratung; modifiziert nach Fiore (Fiore 2000)

\begin{tabular}{|l|l|l|l|}
\hline Dauer der Beratung & $\begin{array}{l}\text { Studien- } \\
\text { arme }\end{array}$ & $\begin{array}{l}\text { Geschätzte Odds } \\
\text { Ratio }(\mathbf{9 5 \%}-\mathbf{K I})\end{array}$ & $\begin{array}{l}\text { Geschätzte Absti- } \\
\text { nenzrate }(95 \%- \\
\text { KI) }\end{array}$ \\
\hline Keine Beratung & 30 & 1,0 & 10,9 \\
\hline Beratungsdauer < 3 min & 19 & $1,3(1,01-1,6)$ & $13,4(10,9-16,1)$ \\
\hline Beratungsdauer 3-10 min & 16 & $1,6(1,2-2,0)$ & $16,0(12,8-19,2)$ \\
\hline Beratungsdauer $>10$ min & 55 & $2,3(2,0-2,7)$ & $22,1(19,4-24,7)$ \\
\hline
\end{tabular}

Da schon eine weniger als 3 Minuten dauernde Beratung die Tabakabstinenzrate steigert (Stead et al. 2008), wird eine solche mit vergleichsweise wenig Aufwand verbundene Minimalintervention durch Mitarbeiter in Gesundheitsberufen in den Leitlinien empfohlen (Andre- 
as et al. 2008, Fiore et al. 2008, Schmidt et al. 2006). So stellte eine entsprechende MetaAnalyse der Cochrane Collaboration unter Hinzunahme von 17 Studien eine signifikante Erhöhung der Rauchstopp-Rate (RR: 1,66; 95 \% - KI: 1,42 - 1,94) durch eine Kurzintervention fest (Stead et al. 2008).

Erste Hinweise auf den Einfluss solch minimaler Interventionen ärztlicherseits offenbarten sich bereits in einer 1968 publizierten Studie, dort im Rahmen eines Hausarzt-Settings: Unter den Rauchern, die von ihrem Hausarzt einen Ratschlag zum Rauchverzicht erhielten, reduzierten nach einer follow-up-Zeit von sechs Monaten insgesamt $33 \%$ ihren Tabakkonsum, während innerhalb der Kohorte ohne eine solche Intervention $9 \%$ der Raucher eine Reduktion vornahmen (Mausner et al. 1968).

\section{Verhaltens- und psychosoziale Interventionen}

Diese in den Formaten Einzeltherapie, Gruppentherapie und Telefonberatung angebotenen Interventionsformen verfolgen eine profundere und intensivere Herangehensweise innerhalb eines umfangreicheren Zeitrahmens mit in der Regel mehreren Sitzungen.

Essentielle Bestandteile sind neben der Vermittlung von Basisinformationen bezüglich der Abhängigkeit vor allem die Förderung von Introspektion, Selbsteinschätzung und Motivation, das Erkennen verleitender Umstände und Situationen, das Erlernen von Selbst- und Impulskontrolle, der Aufbau alternativer Verhaltensweisen, Stressabbau, soziale Unterstützung sowie das Erlernen von Strategien zur Rückfallprophylaxe.

Gruppenprogramme (OR: 1,31; 95 \% - KI: 1,1 - 1,6) und Einzelprogramme (OR: 1,7; 95 \% KI: 1,4 - 2,0) auf kognitiv-verhaltenstherapeutischer Basis wiesen im Rahmen der wissenschaftlichen Evaluation ebenfalls eine hohe Wirksamkeit auf und werden in den Clinical Practice Guidelines empfohlen (Fiore 2000). Im Rahmen von umfassenden Metaanalysen der Cochrane Collaboration konnte dies bestätigt werden (Lancaster und Stead 2005a).

Fazit: Alle aufgeführten Maßnahmen sind nach eingehender wissenschaftlicher Bewertung für sich genommen sehr effektiv und tragen dabei den diversen Komponenten der Tabakabhängigkeit Rechnung. So zielt beispielsweise die pharmakologische Therapie vor allem auf die biologischen Aspekte ab.

Man muss die Tabakabhängigkeit aber in ihrer Gesamtheit betrachten und sich im Idealfall nicht nur auf eine Säule innerhalb der Entwöhnungsmaßnahmen stützen. Diesbezüglich heißt 
es in den Leitlinien, dass die Tabakabhängigkeit vielmehr einen multimodalen Therapieansatz erfordert (Andreas et al. 2008, Fiore et al. 2008, Schmidt et al. 2006). So verspricht die Kombination einer Verhaltenstherapie mit unterstützender individueller Pharmakotherapie die größten Langzeiterfolge (Anthonisen et al. 2005, Mohiuddin et al. 2007).

\subsection{Empfehlungen zur Umsetzung der Tabakentwöhnungsbehandlung}

Trotz der Verfügbarkeit von effektiven Möglichkeiten zur Raucherentwöhnung sind sowohl die Inanspruchnahme als auch die Langzeitabstinenzraten gering.

Zwar gaben bei einer Erhebung im Rahmen einer Studie zur Inanspruchnahme von Hilfen zur Tabakentwöhnung über $70 \%$ der befragten aktuellen Raucher an, in der Vergangenheit mindestens einen ernsthaften Reduktions- oder Aufhörversuch unternommen zu haben. Davon nahmen allerdings nur $19 \%$ eine unterstützende Maßnahme in Anspruch (Meyer et al. 2000). Der am häufigsten genannte Grund für eine Nicht-Inanspruchnahme von Hilfsmitteln lautete bei einer anderen Befragung ähnlichen Hintergrunds: „Ich dachte, ich könne die Rauchreduktion oder den Rauchstopp alleine bewerkstelligen“ (55,2 \%). Etwa ein Viertel war der Meinung, die existierenden Hilfen würden nicht helfen (Gross et al. 2008).

Die Tabakentwöhnung ist dabei nicht von vornherein als frustranes Unterfangen anzusehen. Eine Erhebung in fünf verschieden europäischen Ländern konnte zeigen, dass die Mehrheit der gegenwärtigen Raucher (73,2 \%) mit dem Rauchen aufhören möchte. In Deutschland sagten dabei lediglich 11,1 \%, dass sie auf keinen Fall aufhören möchten (Thyrian et al. 2008).

Diese Zahlen implizieren, dass die Mehrzahl der rauchenden Personen grundsätzlich bereit wäre, den Ausstieg aus der Tabakabhängigkeit zu unternehmen und dementsprechend für eine mögliche begleitende und unterstützende Beratung oder eine andere Raucherentwöhnungsintervention zugänglich wäre.

In den überarbeiteten Clinical Practice Guidelines aus dem Jahre 2008 (Fiore et al. 2008) werden neben der Analyse und Bewertung der zur Verfügung stehenden medikamentösen und verhaltenstherapeutischen Entwöhnungsmaßnahmen ganz konkrete Empfehlungen hinsichtlich der Umsetzung einer adäquaten Raucherentwöhnungsbehandlung ausgesprochen. Diese Empfehlungen basieren auf der systematischen Analyse und Zusammentragung von Erkenntnissen aus mehr als 8700 wissenschaftlichen Arbeiten und haben Leitliniencharakter. 
Konkret heißt es dort, dass jeder Patient in ärztlicher oder generell in gesundheitlicher Behandlung hinsichtlich des Rauchstatus befragt werden sollte mit anschließender Dokumentation dessen. Bei jedem entwöhnungswilligen Raucher sollten dann die entsprechenden Maßnahmen aus Beratung und medikamentöser Therapie zur Anwendung kommen (Fiore et al. 2008). Äquivalente Empfehlungen finden sich auch in der deutschsprachigen Leitlinie (Andreas et al. 2008, Schmidt et al. 2006).

Von entscheidender Bedeutung ist eine effektive und praktikable Umsetzung der empfohlenen Handlungsrichtlinien insbesondere im Hinblick auf eine nachhaltige Integration entsprechender Interventionen und Maßnahmen in die etablierten medizinischen Versorgungsstrukturen. Diesbezüglich und basierend auf der eingehenden Analyse der Studienlage schlägt die Cochrane-Collaboration vor, mit der Tabakentwöhnung bereits während eines Krankenhausaufenthaltes zu beginnen. Die Vorteile: Während des Klinikaufenthaltes ist das Bewusstsein für die eigene Gesundheit besonders vorhanden (,teachable moment"), es liegt eine rauchfreie Umgebung vor und die Ressourcen für eine Entwöhnung sind verfügbar (McBride et al. 2003, Rigotti et al. 2007).

Ferner gibt es Hinweise darauf, dass Raucher empfänglicher für einen ärztlichen Ratschlag zum Rauchstopp sind, sobald dieser an einen medizinischen Befund gekoppelt ist (West et al. 2000). Es zeigen insbesondere diejenigen Raucher eine höhere Motivation zum Rauchverzicht, die ihre körperlichen Symptome mit dem Rauchen in Verbindung bringen (Clark et al. 1999, Walters und Coleman 2002). Dies könnte vor allem bei rauchenden Patienten auf den kardiologischen und pneumologischen Stationen eine Rolle spielen, da der Tabakkonsum hier oft teilweise oder bei manchen Krankheitsbildern sogar in erheblichem Maße zur Pathogenese beiträgt (Pipe et al. 2011). Aber auch unabhängig von der Kausalität des Tabakrauchens an der zur Hospitalisierung führenden Erkrankung bietet der stationäre Aufenthalt offensichtlich einige Vorzüge, Raucher zu identifizieren und schließlich eine Raucherentwöhnung einzuleiten (Emmons und Goldstein 1992, Rigotti et al. 2007).

Zur Realisierung der einschlägigen Maßnahmen einschließlich deren Integration in die Klinikstrukturen eignet sich die Einführung sogenannter „Standard Operating Procedures“ (SOP). Diese finden sich mittlerweile in vielen Bereichen der Medizin (Burnett und Sharlip 2013, Sajdak et al. 2013, Schmid 2012).

Ganz allgemein sieht das Konzept der SOP vor, genaue Handlungsanweisungen und Verhaltensregeln innerhalb bestimmter Prozesse zu definieren, die einen stets identischen Ablauf dieser gewährleisten und so gleichermaßen die Qualität sicherstellen. Im Rahmen einer statio- 
nären Tabakentwöhnung würde so unter anderem spezifiziert werden, welche Aufgaben jeweils von den Pflegekräften oder den ärztlichen Mitarbeitern übernommen werden und wie diese im Einzelnen ausgeführt werden sollen. Dies würde eine standardisierte, leitlinienorientierte und im System fest integrierte Tabakentwöhnungsbehandlung hospitalisierter Raucher gewährleisten.

$\mathrm{Zu}$ dem großen Vorteil einer routinemäßigen Raucherentwöhnungsintervention während stationärer Behandlungen zählt neben den dargestellten günstigen Bedingungen (,teachable moment“, rauchfreie Umgebung etc.) vor allem auch der Zugang, den man auf diesem Wege zu den Rauchern erhält.

Rund $70 \%$ aller Raucher begeben sich jährlich in medizinische Behandlung (Fiore et al. 2008). Es konnte gezeigt werden, dass selbst ein kurzer Ratschlag zum Rauchverzicht während der Arztkonsultation dazu führt, dass ein zwar geringer, aber trotzdem signifikanter Teil der Patienten das Rauchen aufgibt (Chapman 1993). Berücksichtigt man nun die große Anzahl an Rauchern, die jährlich einen Arzt aufsucht, so erweist sich der potentielle Effekt einer solchen, selbst minimalen Intervention auf die öffentliche Gesundheit als beträchtlich.

Im Jahre 2012 gab es in Deutschland insgesamt 18,6 Millionen stationäre Behandlungen mit einer durchschnittlichen Liegedauer von 7,6 Tagen (Statistisches Bundesamt 2013). Jeder Raucher, der hier nicht zumindest eine kurze Ausstiegsberatung oder einen minimalen Anstoß zum Rauchverzicht erhalten würde, ungeachtet einer wünschenswerten individuellen und patientenadaptierten Unterstützung im Idealfall, wäre eine verpasste Gelegenheit (,,missed opportunity $\left.{ }^{6}\right)$. 


\subsection{Entwöhnung bei hospitalisierten Patienten - aktuelle Versorgungslage}

Allen Empfehlungen zum Trotz gibt es Faktoren, die einen limitierenden Einfluss auf eine umfassende und adäquate Versorgung von Rauchern haben. Eine fehlende Bereitschaft zum Rauchverzicht auf Seiten der Raucherinnen und Raucher mag eines dieser Hindernisse darstellen. Nach den Ergebnissen von Thyrian weist in Deutschland allerdings nur ein geringer Anteil an Rauchern eine solche vollständig ablehnende Haltung auf, während die große Mehrheit für eventuelle Entwöhnungsmaßnahmen grundsätzlich zugänglich wäre (Thyrian et al. 2008). Die Hindernisse sind demzufolge nicht unbedingt nur auf der Nachfrageseite, also bei entwöhnungsunwilligen Raucherinnen und Rauchern zu verzeichnen.

Insbesondere und gerade auf Seiten medizinischer Strukturen und des medizinischen Personals konnten in mehreren Studien gewisse Limitationen nachgewiesen werden, die sich in negativer Weise auf die Versorgungslage hospitalisierter Raucher auswirken.

Dazu zählt eine mangelnde Umsetzung und Priorisierung von Raucherentwöhnungsberatungen. Eine an der Universitätsklinik Göttingen durchgeführte Erhebung ergab zwar, dass mit 93,5\% die Mehrheit der befragten Ärztinnen und Ärzte es für sinnvoll und wichtig erachtet, Rauchern einen Ratschlag zum Rauchverzicht zu erteilen (Raupach et al. 2011). Die Durchführung entsprechender Entwöhnungsinterventionen wurde hingegen nur von 27,3\% der befragten ärztlichen Mitarbeiter angegeben.

Ein weiterer bedeutender Aspekt auf Seiten des medizinischen Personals mit Auswirkung auf die Versorgungslage rauchender Patienten stellen Defizite im Wissen um eine angemessene Tabakentwöhnungsbehandlung sowie Fehleinschätzungen bezüglich der Effektivität der zur Verfügung stehenden therapeutischen Maßnahmen dar.

Die bereits erwähnte Studie an der Göttinger Universitätsklinik zeigte in diesem Zusammenhang, dass nicht nur unter den befragten Patienten, sondern auch ärztlicherseits die tabakrauchassoziierten Risiken für die Entwicklung bestimmter Krankheiten unterschätzt wurden (Raupach et al. 2011). Ferner von Ärzten und Patienten gleichermaßen unterschätzt wurde die Wirksamkeit der diversen Tabakentwöhnungsmaßnahmen. Beide Gruppen führten als effektivstes Mittel zur Abstinenzerlangung die „Willenskraft“ am häufigsten auf (Raupach et al. 2011). Eine ähnliche Fehleinschätzung konnte in einer Befragung deutscher Medizinstudenten nachgewiesen werden (Raupach et al. 2013).

Ein wesentlicher zugrundeliegender Aspekt dessen stellt die Vermittlung entsprechender Inhalte während der universitären Medizinerausbildung dar. Eine Umfrage an über 20 medizini- 
schen Fakultäten in Deutschland zeigte, dass das Thema „Rauchen“ mit durchschnittlich 135 Minuten weniger umfangreich im Curriculum verankert ist als andere Erkrankungen wie beispielsweise der Diabetes mellitus (353 Minuten) oder die arterielle Hypertonie (275 Minuten) (Strobel et al. 2012). Folglich gaben über $80 \%$ der Medizinstudenten im fünften Jahr an zu wissen, wie man die beiden letzteren Erkrankungen behandelt. Bei der Frage nach der Behandlung der Tabakabhängigkeit äußerten $20 \%$ der befragten Studenten, diesbezüglich über gute Kenntnisse zu verfügen. Insgesamt waren $60 \%$ der Meinung, man solle die Lehre auf diesem Gebiet intensivieren (Strobel et al. 2012). Eine Erweiterung dieser Studie mit Befragung der Studierenden im letzten Jahr der Ausbildung zeigte nach wie vor bestehende Defizite (Anders et al. 2013).

Ähnliche Ergebnisse hinsichtlich der Vermittlung der Inhalte der Tabakabhängigkeit einschließlich deren Therapie während der Medizinerausbildung konnten auch in anderen Ländern reproduziert werden (Djalalinia et al. 2011, Grassi et al. 2012, Roddy et al. 2004, Springer et al. 2008). So ergab eine Erhebung unter britischen Assistenzärzten im ersten Berufsjahr, dass sich $60 \%$ der Absolventen nicht in der Lage fühlten, eine leitliniengerechte Tabakentwöhnungsbehandlung durchzuführen (Roddy et al. 2004, Springer et al. 2008). Etwa $17 \%$ fühlten sich gut vorbereitet, Ratschläge bezüglich einer Nikotinersatztherapie geben zu können, bei der Therapie mit Bupropion gaben dies lediglich $5 \%$ der befragten Assistenzärzte an.

Die dargestellten Erkenntnisse dieser vordergründig qualitativen Defizite stellen entscheidende Limitationen und Barrieren auf dem Weg zu einer angemessenen und leitliniengerechten Versorgung rauchender Patienten dar.

In dem Positionspapier der Canadian Cardiovascular Society wird diesem Umstand Rechnung getragen; entsprechend werden dezidierte Forderungen an die behandelnden Ärzte, insbesondere an die Kardiologen, gestellt: So solle jeder Raucher in einem medizinischen Setting nicht nur identifiziert werden, sondern sei es darüber hinaus die Pflicht und Verantwortung eines jeden behandelnden Arztes, einen eindeutigen Ratschlag zum Rauchverzicht zu kommunizieren und eine Tabakentwöhnung zu initiieren. Dabei solle der Arzt vertraut sein mit den Prinzipien der Tabakabhängigkeit sowie deren pharmakologischer Therapie, ebenso wie er vertraut sei mit der Therapie der arteriellen Hypertonie oder der Hypercholesterinämie (Pipe et al. 2011).

Ganz allgemein betrachtet kann also ein entsprechendes Training der medizinischen Kräfte und folglich eine Änderung des Verhaltens in Richtung vermehrter leitlinienorientierter Ent- 
wöhnungsberatungen maßgeblich zur Verbesserung der Versorgungslage von Rauchern beitragen.

In diesem Zusammenhang soll das sogenannte „COM-B“- Modell vorgestellt werden (Michie et al. 2011). Es charakterisiert und betrachtet alle Parameter, die das Verhalten einer Person determinieren bzw. einen Einfluss darauf haben. Gleichzeitig lassen sich auch Erkenntnisse hinsichtlich der Entwicklung von Interventionen zur Verhaltensänderung ableiten.

Abb. 2: „COM-B“ - Modell, modifiziert nach Michie et al. 2011

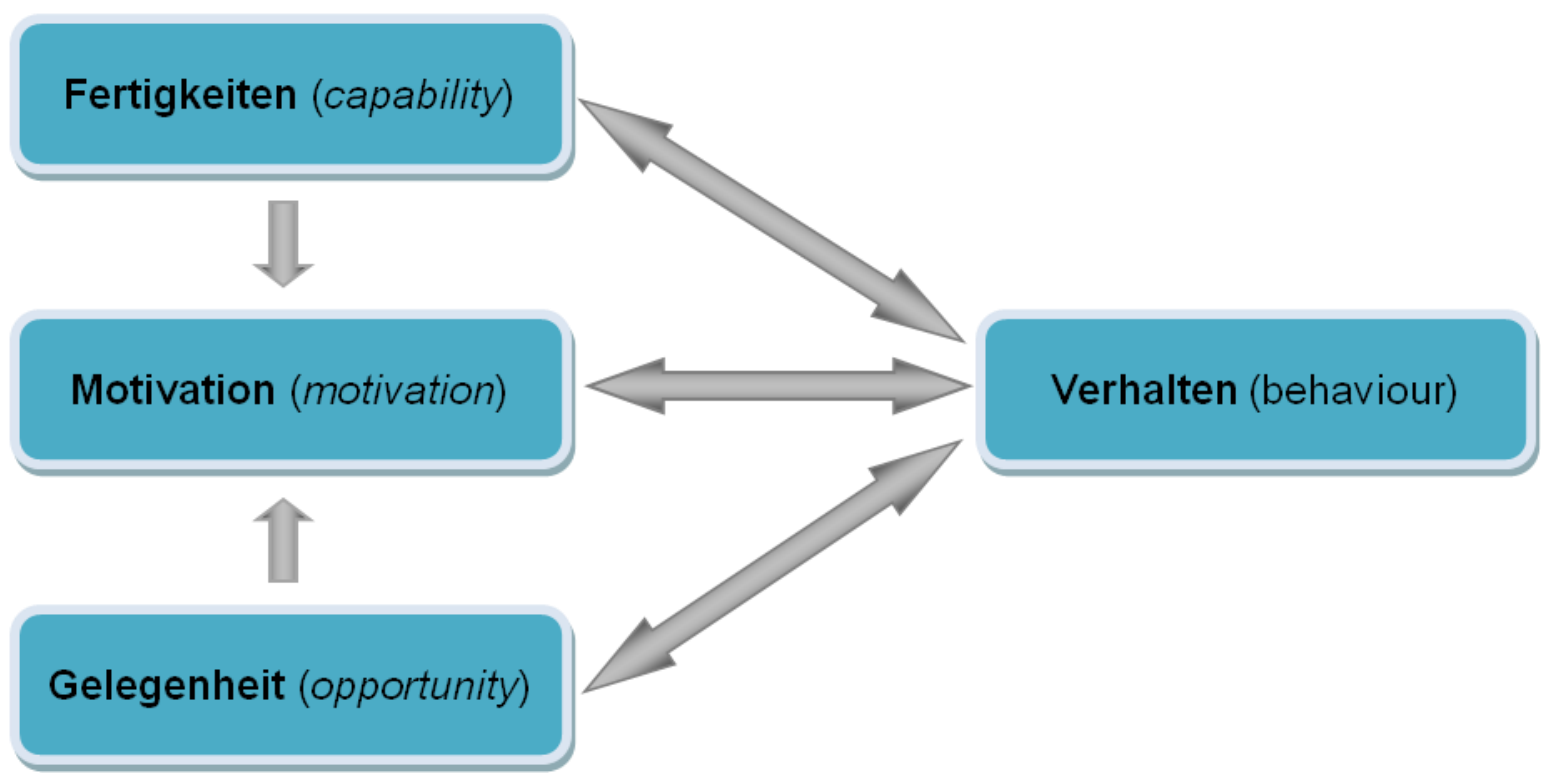

Nach dem „COM-B““- Modell (siehe auch Abb. 2) wird das Verhalten (behaviour) einer Person im Wesentlichen von drei verschiedenen Elementen beeinflusst:

Einerseits von den Fertigkeiten und Fähigkeiten (capability), ein bestimmtes Verhalten ausführen zu können. Dazu zählen die physischen Voraussetzungen ebenso wie die mentalen, beispielsweise also das Wissen oder die Kenntnisse darüber. Andererseits spielt die Gelegenheit (opportunity) eine grundlegende Rolle. Sie beschreibt die äußeren Faktoren, die ein Verhalten fördern oder gar hindern kann. Untergliedert in physische und soziale Faktoren sind hier unter anderem materielle Ressourcen, eine ausreichende Zeitkomponente sowie die kulturellen Rahmenbedingungen gemeint. Schließlich wird das Verhalten von der Motivation (motivation) beeinflusst. Neben Absichten, Plänen und Wünschen fallen hierunter auch die größtenteils unbewusst ablaufenden Aspekte wie Emotionen und Impulse. Es besteht dabei eine wechselseitige Beeinflussung der jeweiligen Parameter. 
Eine Anwendung dieses Modells auf die zuvor genannten Defizite im Bereich des Wissens und der Kompetenzen bei der Raucherentwöhnung unter den medizinischen Kräften verdeutlicht, dass in diesem Fall der limitierende Faktor dem Gebiet der Fähigkeiten (capability) zuzuordnen ist und so einem wünschenswerten, leitliniengerechten Verhalten entgegensteht. In einem anderen Szenario wäre es ebenfalls denkbar, dass die Ärzte zwar über entsprechende Kompetenzen und Fertigkeiten verfügen und darüber hinaus auch gewillt sind (motivation), eine Beratung von Rauchern durchzuführen, allerdings aufgrund von Zeitmangel keine Gelegenheit (opportunity) dazu haben. Auch hier würde das gewünschte Verhalten nicht eintreten. Insgesamt trägt das Modell dazu bei, die einem bestimmten Verhalten zugrundeliegenden Faktoren zu identifizieren, um sie in einem zweiten Schritt im Falle ihrer defizitären Ausprägung positiv zu beeinflussen.

\subsection{Ziele der Studie}

Die Deutsche Gesellschaft für Kardiologie ebenso wie die Deutsche Gesellschaft für Pneumologie und Beatmungsmedizin stellen neben vielen anderen, zum Teil länderübergreifenden Fachgesellschaften die Notwendigkeit und Bedeutung einer Tabakentwöhnung nicht nur aus primärpräventiver Sicht, sondern vor allem bei Patienten mit kardiovaskulären Erkrankungen heraus (Dietz und Rauch 2003, Perk et al. 2012, Pipe et al. 2011). Gemäß der Leitlinien wird gerade in letzterem Fall die Einleitung entsprechender Entwöhnungsinterventionen bereits während der Hospitalisierung der Raucher empfohlen (Fiore et al. 2008, Rigotti et al. 2007). Die Umsetzung und Integration der empfohlenen Handlungsrichtlinien in die existierenden Klinikstrukturen lässt sich mit der Einführung von SOPs realisieren.

Diverse Studien aus Deutschland zeigen jedoch bestehende Limitation und Defizite, die einer adäquaten Tabakentwöhnungsbehandlung im Wege stehen (Anders et al. 2013, Raupach et al. 2011, Strobel et al. 2012).

Primäres Ziel der hier vorgestellten Studie war die Optimierung der Versorgungsqualität rauchender Patienten in der Abteilung für Kardiologie und Pneumologie der Universitätsmedizin Göttingen (UMG).

Zu Beginn des Projektes erfolgte zunächst die Evaluation der gegenwärtig auf den kardiologischen Stationen angewandten und praktizierten Maßnahmen in Bezug auf die Tabakentwöhnung rauchender Patienten. Nach Ablauf dieser initialen Kontrollphase wurden SOPs zum 
leitlinienkonformen Management der Raucher implementiert und von Maßnahmen zur Mitarbeiter-Fortbildung flankiert. Es folgte eine weitere Phase der Evaluation entsprechender Maßnahmen.

Die auf diese Weise gewonnenen Daten sollten Rückschlüsse ermöglichen auf die durch die Intervention induzierte Beeinflussung folgender Aspekte:

1) Die Interventionshäufigkeit bei Rauchern durch ärztliche und pflegerische Mitarbeiter/innen

2) Zufriedenheit der Patienten mit der Entwöhnungsberatung

3) Dokumentation des Rauchstatus sowie einer Entwöhnungs-Intervention

4) Die Bereitschaft der betroffenen Patienten, das Rauchen aufzugeben

5) Die Zahl der Raucher, welche das Angebot der klinikeigenen Tabakentwöhnungsambulanz in Anspruch nehmen

6) Die zusätzliche Arbeitsbelastung der Mitarbeiter

Nach Auswertung der entsprechenden Daten wurde in einem darauffolgenden zweiten Teil dieser Studie die umgesetzte SOP zu Optimierungs- und Überarbeitungszwecken evaluiert. Im Rahmen von Einzelinterviews mit den auf den Studienstationen eingesetzten Mitarbeitern sollten Erfahrungen, Limitationen und optimierungsbedürftige Prozesse aufgedeckt und thematisiert werden mit dem Ziel, Maßnahmen zur Sicherung einer nachhaltigen und leitliniengerechten Versorgung hospitalisierter Raucher abzuleiten. 


\section{Material und Methoden}

\subsection{Studiendesign}

Bei dem hier vorgestellten Projekt handelte es sich um eine prospektive Interventionsstudie auf den kardiologischen Stationen der Universitätsmedizin Göttingen (UMG). Dabei kamen sowohl quantitative als auch qualitative Forschungsmethoden zum Einsatz.

Im ersten Teil der Studie (Phase I) sollte zunächst die Qualität der Patientenversorgung hinsichtlich der Suchterkrankung Rauchen auf den beiden kardiologischen Normalstationen 2021 und 2022 sowie der Intermediate Care (IMC) - Station 1025 evaluiert werden. Die Fragebogen-gestützte quantitative Datenerhebung erfolgte über einen Zeitraum von insgesamt drei Monaten (Februar - April 2011).

Im Anschluss an diese Kontrollphase wurden Standard Operating Procedures (SOP) für das Management rauchender Patienten eingeführt. Begleitend fand eine Schulung aller auf diesen Stationen eingesetzten ärztlichen Mitarbeiter sowie der dort tätigen Pflegekräfte statt.

In dem darauffolgenden zweiten Teil der Studie (Phase II) wurde auf diesen Stationen, analog zum ersten Studienabschnitt, die Fragebogen-gestützte Datenerhebung zur Ermittlung der Versorgungsqualität der rauchenden Patienten fortgeführt. Diese sogenannte Interventionsperiode erstreckte sich ebenfalls über drei Monate (Mai - Juli 2011).

In beiden Phasen wurden neben den Patienten auch die pflegerischen und ärztlichen Mitarbeiter/innen mit Hilfe von Fragebogen befragt. In einem Vorher-Nachher-Design mit dem Vergleich der Ergebnisse aus der initialen Kontrollperiode mit denen der anschließenden Interventionsperiode wurden Unterschiede in der Versorgung untersucht, die durch Implementierung der SOP sowie der strukturierten Schulungsmaßnahme des Personals induziert worden waren.

Im Anschluss an die dargestellte quantitative Erhebung folgte auf der Basis der Auswertung der so generierten Daten die Durchführung von Einzelinterviews mit den ärztlichen und pflegerischen Mitarbeitern, die während der Interventionsperiode auf den jeweiligen kardiologischen Stationen eingesetzt gewesen waren. Dieser qualitative Forschungsansatz als zweiter wesentlicher Bestandteil des Projektes diente der Analyse fehlerhafter Prozesse sowohl innerhalb der eigentlichen SOP als auch bei deren Anwendung im Stationsalltag zum Zwecke der anschließenden Optimierung. 
Eine Übersicht über den Aufbau der gesamten Studie mit ihren einzelnen Elementen sowie dem zeitlichen Verlauf liefert Abbildung 3.

Abb. 3: Aufbau der Studie
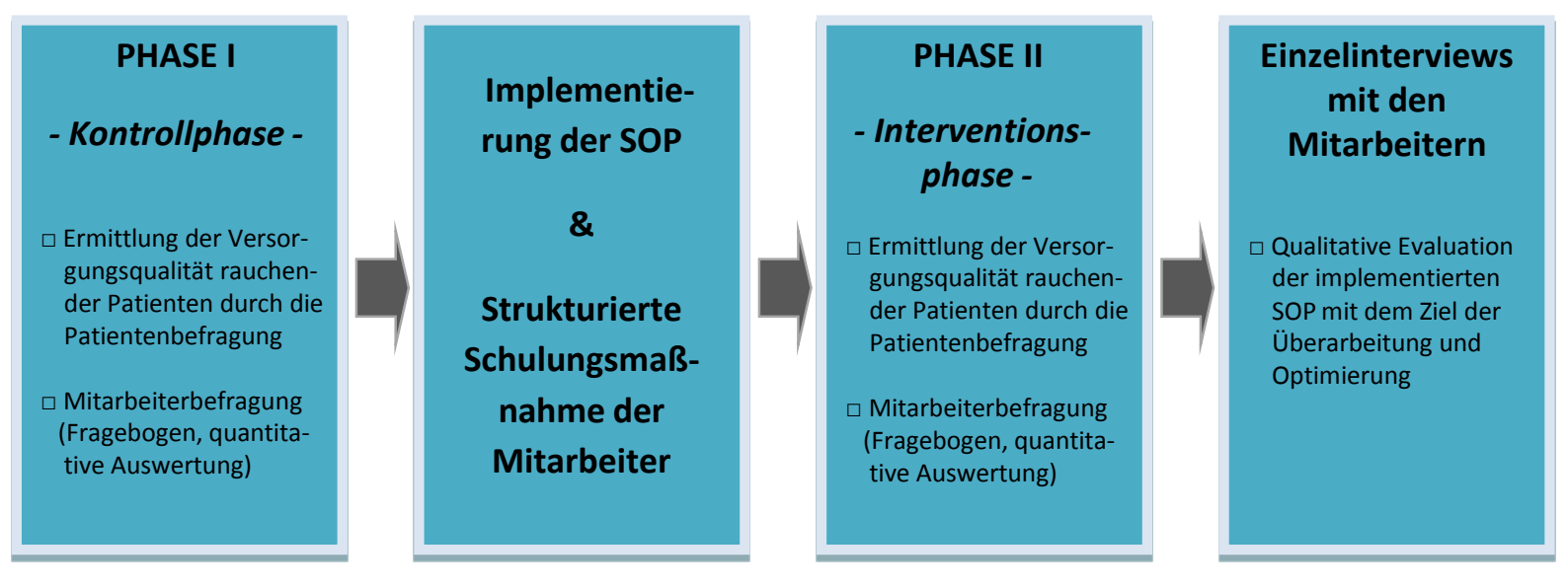

\subsection{Probandenrekrutierung und Datensammlung}

\subsubsection{Patientenbefragung}

Als Studienteilnehmer kamen alle rauchenden Patientinnen und Patienten in Frage, die sich zur elektiven Aufnahme auf einer der kardiologischen Stationen 2021, 2022 oder 1025 vorstellten oder per haus-interner Verlegung dorthin gelangten.

Im Falle einer geplanten stationären Aufnahme wurde der Patient bei der Anmeldung im Ebenenbüro um sein Einverständnis zur Studienteilnahme gebeten. Die Mitarbeiterinnen und Mitarbeiter des Ebenenbüros wurden entsprechend instruiert, alle vorstelligen Patienten nach ihrem Rauchstatus zu fragen, um so die Raucherinnen und Raucher zu identifizieren. Diese erhielten dann ein einseitiges Informationsblatt und einen Vordruck der Einverständniserklärung (Dokumente im Anhang). Nichtraucher und Nichtraucherinnen wurden nicht eingeschlossen.

Zum Einschluss in die aktuelle Studie mussten die Patienten sowohl volljährig als auch einwilligungsfähig sein. Die Einwilligungsfähigkeit war gegeben, wenn ein Patient der deutschen Sprache in Schrift und Wort mächtig war und die Informationsschrift sowie die Einverständniserklärung verstand. Patienten mit unterschriebener Einverständniserklärung wurden noch im Ebenenbüro darum gebeten, den Fragebogen vor Intervention auszufüllen (siehe Kapitel 2.4). Die ausgefüllten Dokumente wurden im Ebenenbüro behalten und im Verlauf vom Autor dieser Arbeit abgeholt. 
Patienten, die per haus-interner Verlegung auf eine der Stationen 2021, 2022 oder 1025 gelangten, wurden durch die Pflegekräfte im Ebenenbüro registriert und konnten somit im Aufnahmebuch identifiziert werden. Am Morgen nach der Verlegung auf eine der genannten kardiologischen Stationen wurden diese Patienten vom Autor dieser Arbeit aufgesucht und nach ihrem Rauchverhalten befragt. Alle rauchenden Patienten wurden dann zur Teilnahme an der Studie eingeladen und aufgeklärt. Nach erfolgter Einwilligung wurden die Patienten gebeten, den Fragebogen vor Intervention auszufüllen.

Um fortan die Anonymität aller Studienteilnehmer zu wahren, wurde jeder Fragebogen mit einer Codenummer versehen. Diese Zuordnung erfolgte - je nach Aufnahmeart - entweder im Ebenenbüro oder direkt auf der Station. Zur Identifikation wurde eine fortlaufend nummerierte Liste verwendet. Die Patientenaufkleber von den Studienteilnehmern wurden jeweils neben die nächste freie Nummer in dieser Liste geklebt. Die Datenspeicherung erfolgte im weiteren Verlauf anhand der entsprechenden Codenummer und nicht mehr anhand persönlicher Daten. Alle Fragebogen wurden lediglich mit der jeweiligen Codenummer gekennzeichnet. Die Zuordnungsbogen mit den Patientenaufklebern wurden vor der Datenanalyse vernichtet.

Zur Evaluation pflegerischer und/oder ärztlicher Interventionen zur Tabakentwöhnung während des stationären Aufenthaltes wurde ein Fragebogen nach Intervention entworfen (siehe Kapitel 2.4). Jeweils am Vorabend des Entlassungstages wurde jedem Patienten, der am Aufnahmetag als Raucher identifiziert wurde und der Studienteilnahme zugestimmt hatte, dieser zweite, pseudonymisierte Fragebogen vorgelegt.

Um sicherzustellen, dass jeder Studienteilnehmer den Fragebogen nach Intervention ausfüllen konnte, informierte sich der Autor dieser Arbeit für die Dauer der Studie jeweils am Vortag über die anstehenden Entlassungen des folgenden Tages, um dann das Abschlussgespräch mit dem Patienten zu planen, in dem der Fragebogen ausgefüllt wurde. Anhand der oben erwähnten Zuordnungsliste mit den jeweiligen Codenummern konnte nun auch der abschließende Fragebogen mit der entsprechenden Nummer versehen werden. So wurde für die spätere Auswertung gewährleistet, dass Aufnahme- und Entlassungsfragebogen eines Patienten die jeweils gleiche Codenummer hatten. 


\subsubsection{Mitarbeiterbefragung}

Weiterer Gegenstand der dargestellten Studie war die Befragung aller ärztlichen und pflegerischen Mitarbeiter, die während der beiden Projektphasen auf den Stationen 2021, 2022 und 1025 eingesetzt waren.

Die Erhebung erfolgte ebenfalls mit Hilfe von Fragebogen. In Bezug auf die zeitliche Einordnung fand der erste Teil dieser Befragung innerhalb der dreimonatigen Phase vor Implementierung der SOP (Phase I) statt, während der zweite Teil in den darauffolgenden drei Monaten (Phase II) durchgeführt wurde. Die jeweiligen Fragebogen (Erhebungsbogen Pflege vor Intervention sowie Erhebungsbogen Pflege nach Intervention) werden in Kapitel 2.4 dargestellt.

Die Befragung der pflegerisch tätigen Kräfte wurde im Vorhinein sowohl auf Mitarbeiterbesprechungen und Schichtübergaben als auch per E-Mail durch die Ebenenleitung kommuniziert. Ärztlicherseits erfolgte dies in entsprechender Weise durch den Studienleiter PD Dr. Tobias Raupach und die Oberärzte.

Unmittelbar vor Distribution der Erhebungsbogen in die jeweiligen Postfächer der Mitarbeiter erfolgte durch den Autor dieser Arbeit die Kennzeichnung der einzelnen Bogen mit einer Codenummer, um fortan eine anonyme Verwendung der personenbezogenen Daten zu gewährleisten. Die Zuordnung wurde anhand einer Liste vorgenommen, auf der die Namen sämtlicher Mitarbeiter einschließlich einer jeweils nebenstehenden Codenummer aufgeführt waren. Diese wurde auf dem Fragebogen vermerkt, welcher unmittelbar in das entsprechende Postfach des Mitarbeiters sortiert wurde. Ausgefüllte Fragebogen waren in ein speziell dafür vorgesehenes Fach einzuwerfen und wurden dort vom Autor der Arbeit wieder eingesammelt. Analog zu dieser Vorgehensweise wurde auch bei der Mitarbeiterbefragung in Phase II vorgegangen, wobei dieselbe Liste mit den Codenummern für die Zuordnung und Kennzeichnung verwendet wurde. Die weitere Datenspeicherung erfolgte ausschließlich anhand dieser Nummern.

Die Distribution der Erhebungsbogen (Erhebungsbogen Pflege nach Intervention) wurde dabei in der zweiten Hälfte von Phase II begonnen, um eine ausreichende Einführung und Integration der SOP in die Stationsarbeit zu gewährleisten. 


\subsubsection{Darstellung der Mitarbeiter-Interviews}

Zur näheren Untersuchung der Determinanten des Verhaltens der pflegerischen und ärztlichen Mitarbeiter während der Implementierungsphase wurden Einzelinterviews geführt und mit Bezug auf das oben beschriebene „COM- $B$ “- Modell ausgewertet. Zudem dienten die Interviews der Analyse etwaiger Probleme bei der Konzeption sowie der praktischen Umsetzung der SOP; die so gewonnenen Daten sollten die Grundlage für eine Überarbeitung der SOP liefern. In den Einzelinterviews wurden Erfahrungen und Schwierigkeiten bei der Umsetzung der SOP, die Einstellung zur SOP und der Beratung rauchender Patienten während des stationären Klinikaufenthaltes im Allgemeinen, die Integration all dessen in den Arbeitsalltag sowie Verbesserungsvorschläge, auch in Bezug auf die Trainingsmaßnahme, thematisiert.

Um die Besprechung aller Aspekte in den Interviews zu gewährleisten, mussten die einzelnen Mitarbeiter frei sein von etwaigen Einflüssen durch andere Mitarbeiter oder Vorgesetzte. Im Rahmen von Gruppengesprächen oder Gruppeninterviews hätte die Gefahr bestanden, dass gewisse Ansichten und Aspekte aufgrund der Tendenz zur sozialen Erwünschtheit nicht kommuniziert würden. Aus diesem Grund wurden die Einzelinterviews den ebenfalls zu den qualitativen Methoden zählenden Fokusgruppen-Diskussionen vorgezogen.

Die Einzelinterviews wurden von einer unabhängigen und in Bezug auf qualitative Forschungsmaßnahmen entsprechend geschulten Person durchgeführt. Diese Aufgabe wurde von Dipl.- Psych. Sarah Schiekirka übernommen, die als Koordinatorin der Medizindidaktik zwar an der Universitätsmedizin Göttingen arbeitet, aber nicht in die Patientenversorgung involviert ist. Frau Schiekirka war weder an der Entwicklung und Implementierung der SOP noch an den Mitarbeiterschulungen beteiligt. Auf diese Weise sollte eine potenzielle Einflussnahme auf das Antwortverhalten der Interviewten ausgeschlossen werden.

Die Einzelinterviews umfassten einen zeitlichen Rahmen von ungefähr 30 Minuten und wurden als sogenannte Leitfadeninterviews geführt. Dabei wurde der Interviewerin ein zuvor zusammengestellter Fragenkatalog (siehe Kapitel 2.4.3) mit allen in Bezug auf das Forschungsthema relevanten Inhalten zur Verfügung gestellt. Dieser Leitfaden diente in erster Linie der Standardisierung der Gespräche. Die Reihenfolge der einzelnen Fragen ebenso wie die exakte Formulierung waren nicht strikt vorgeschrieben.

Die jeweiligen Fragen wurden so gestellt, dass die interviewten Probanden möglichst ausführlich und offen antworten konnten, wozu sie ausdrücklich ermuntert wurden. Vorgegebene 
Antwortmöglichkeiten gab es nicht. Durch gezieltes Nachfragen konnten Teilaspekte bei Bedarf vertieft werden.

Die kompletten Interviews wurden akustisch-elektronisch aufgezeichnet und anschließend wörtlich transkribiert. Die Transkription wurde dabei von einer unabhängigen dritten Person, die in keiner Weise in Zusammenhang mit der Stationsarbeit, der Entwicklung und Implementierung der SOP sowie der Trainingsmaßnahme stand, vorgenommen.

Mit Ausnahme des Geschlechts und der jeweiligen Berufsgruppe enthielten die Transkripte der Interviews keine persönlichen Daten der Probanden. Die weitere Auswertung der Daten erfolgte ausschließlich anhand der Abschriften. Unmittelbar nach der erfolgten Transkription wurde das in elektronischer Form gespeicherter Interview gelöscht. Eine nachträgliche $\mathrm{Zu}$ ordnung oder Rückverfolgung der Probanden war nicht möglich.

Als Teilnehmer für die dargestellten Einzelinterviews kamen alle ärztlichen und pflegerisch tätigen Mitarbeiter in Frage, die auf den entsprechenden Studienstationen während der dreimonatigen Interventionsperiode (Phase II) eingesetzt waren. Die Teilnahme an dem Interview war freiwillig. Die Einladung zur Interview-Teilnahme wurde zusammen mit einem Informationsblatt an die in Frage kommenden Ärztinnen und Ärzte per E-Mail versandt. Die Pflegekräfte wiederum wurden in Absprache mit der Pflegedienstleitung durch die jeweiligen Stationsleitungen von der Möglichkeit der Teilnahme in Kenntnis gesetzt. Auf allen betreffenden Stationen wurden Teilnehmerlisten ausgehängt, in die sich interessierte Mitarbeiter eintragen konnten. Der genaue Interviewtermin wurde dann von der Interviewerin mit dem Interessenten individuell verabredet.

Unmittelbar vor dem Interview erhielten die Probanden ein einseitiges Informationsblatt sowie die Einwilligungserklärung (Dokumente im Anhang). Letztere musste vor Beginn des Interviews von allen Probanden unterzeichnet werden. 


\subsection{Intervention}

Nach Beendigung der initialen dreimonatigen Kontrollperiode (Phase I) fand auf den Stationen 2021, 2022 und 1025 die im Folgenden dargestellte Modifikation der Prozessabläufe (Standard Operating Procedures) sowie die strukturierte Trainingsmaßnahme für die jeweiligen ärztlichen und pflegerischen Mitarbeiter statt:

\section{a) Standard Operating Procedures}

Im Rahmen des Aufnahmegesprächs sollte von den Pflegekräften zunächst der Rauchstatus aller Patienten dokumentiert werden. Aktivrauchen sollte durch das Ziehen des grauen Reiters in der Pflege-Planette kenntlich gemacht werden. Dies diente bei der späteren Datensammlung der Identifikation von rauchenden Patienten und stellte insofern eine studienbezogene Maßnahme und keinen spezifischen Teil der SOP dar. Ferner sollte die zuständige Pflegekraft den Namen des rauchenden Patienten an der Stationstafel mit einem Kreuz versehen. Diese Wandtafeln befinden sich in der UMG auf allen Stationen jeweils in den Stationszimmern und sind nur den ärztlichen und pflegerischen Mitarbeitern zugänglich. Die Wandtafel nimmt eine zentrale Rolle bei den täglichen Abläufen ein, weil alle Pflege-Übergaben mit ihrer Hilfe moderiert werden.

Im Rahmen der pflegerischen Aufnahme der rauchenden Patienten sollten die Pflegekräfte das Rauchen idealerweise bereits kurz thematisieren. Dies sollte nicht in dem Umfang geschehen, wie es im weiteren Verlauf des stationären Aufenthalts ärztlicherseits erfolgen sollte. In wenigen Sätzen sollte der positive Effekt einer Entwöhnung für den Patienten hervorgehoben werden sowie Hilfe diesbezüglich angeboten werden, unter anderen mit dem Verweis auf eine mögliche medikamentöse Unterstützung sowie die klinikeigene Raucherentwöhnungsambulanz.

Die Markierung des Patientennamens an der Wandtafel diente als Hinweis für das ärztliche Personal, dass bei gekennzeichneten Patienten die Indikation für eine Tabakentwöhnungsberatung bestand. Diese Beratung musste möglichst zeitnah innerhalb der ersten drei Tage nach Aufnahme des Patienten stattfinden.

In Anlehnung an internationale Empfehlungen sollte das Gespräch auf der Grundlage des 5 ASchemas (ask, advise, assess, assist, arrange) erfolgen, welches bereits in Kapitel 1.5 bei der Vorstellung der Tabakentwöhnungsmaßnahmen dargestellt wurde (Fiore et al. 2008). 
Essentielle Bestandteile dabei sind die Erhebung der Raucheranamnese, das Anraten eines Rauchverzichts unter Hervorhebung der positiven Auswirkungen auf die Gesundheit des Patienten sowie die Ermittlung der individuellen Aufhörbereitschaft (siehe Tab. 2, Kapitel 1.5). Dem Patienten sollte explizit die Hilfe von ärztlicher und pflegerischer Seite zugesichert werden. Dies beinhaltet die Aufklärung über die therapeutischen Möglichkeiten der Tabakentwöhnung, insbesondere auch die der medikamentösen Therapie. Sofern vom Patienten gewünscht, sollten die entsprechenden Anordnungen getroffen werden. Eventuell auftretende Fragen bezüglich der Applikation oder der Handhabung der Nikotinersatzpräparate sollten durch die Pflegerinnen und Pfleger auf Station geklärt werden. Außerdem sollte die fakultative Unterstützung durch die klinikeigene Raucherentwöhnungsambulanz kommuniziert werden und gegebenenfalls eine Anbindung erfolgen. In diesem Fall war es die Aufgabe der Pflegekräfte, einen Termin in der Entwöhnungsambulanz zu vereinbaren.

Die SOP wurde, für alle Mitarbeiter gut sichtbar, auf den betreffenden Stationen ausgehängt. In tabellarischer Form waren die jeweiligen Aufgaben und Handlungsrichtlinien sowohl für das ärztliche als auch das pflegerische Personal aufgeführt, ergänzt durch Empfehlungen zur medikamentösen Begleittherapie der Raucher sowie ein Informationsblatt zur Nikotinersatztherapie. Die entsprechenden Dokumente befinden sich im Anhang dieser Arbeit.

\section{b) Trainingsmaßnahme}

Das zweite wesentliche Element der Intervention stellte die strukturierte Trainingsmaßnahme der auf den Stationen 2021, 2022 und 1025 tätigen ärztlichen und pflegerischen Mitarbeiter dar. Zeitlich lag die Schulung zwischen der initialen Kontrollperiode (Phase I) und der sich anschließenden Interventionsperiode (Phase II).

Um möglichst vielen Mitarbeitern die Gelegenheit einer Teilnahme daran zu ermöglichen, wurden in Absprache mit den für die jeweiligen Pflegekräfte zuständigen Ebenenleitern zwei Schulungstermine an verschiedenen Tagen festgelegt. Drei Wochen vor Beginn der Trainingsmaßnahme wurden in den Stationszimmern Listen ausgehängt, in die sich die Mitarbeiter für einen der jeweiligen Termine eintragen konnten. Parallel wurden die Pflegekräfte vor allem durch die Ebenenleiter von der Schulung in Kenntnis gesetzt. Dies geschah durch Aushänge auf Station, per E-Mail an die einzelnen Mitarbeiter sowie bei Besprechungen und Schichtübergaben. 
Die Inkenntnissetzung der ärztlichen Mitarbeiter erfolgte durch die Disseminierung der SOP über den E-Mail-Verteiler der Abteilung Kardiologie \& Pneumologie. Zudem wurde die SOP auf einer Nachmittagsbesprechung der Abteilung vorgestellt. Die Oberärzte der Stationen wurden über das Projekt informiert und gebeten, die Umsetzung der SOP zu unterstützen.

Die Schulungen fanden am 26. und 27.04.2011 im Besprechungsraum der Inneren Medizin auf Ebene 3 im Bettenhaus 2 der Universitätsklinik Göttingen statt.

Zu Beginn der Trainingsmaßnahme wurden die an dem Projekt beteiligten sowie die speziell an der Schulung partizipierenden Personen durch den Schulungs- und Studienleiter PD Dr. T. Raupach vorgestellt. Es handelte sich im Einzelnen um Dipl.-Psych. Peter Lindinger, unter anderem tätig für das WHO-Kollaborationszentrum für Tabakkontrolle des Deutschen Krebsforschungszentrum Heidelberg, Dipl.-Päd. Anna Ludwig, Kursleiterin der klinikeigenen Raucherentwöhnungsambulanz sowie der Autor dieser Arbeit.

Im ersten Abschnitt der Schulung wurde eine Einführung in das Thema „Rauchen und Tabakentwöhnung“ gegeben und die Standard Operating Procedures vorgestellt. Der durch eine 30minütige PowerPoint-Präsentation gestützte Vortrag durch den Schulungsleiter vermittelte die folgenden Inhalte:

Zunächst wurden einige epidemiologische Daten bezüglich des Rauchens präsentiert, insbesondere der Zusammenhang des Rauchens mit kardiovaskulären Erkrankungen sowie Zahlen zur tabakrauchassoziierten Mortalität. Anhand eines Beispiels wurde den Teilnehmern die zugrundeliegenden Mechanismen der Nikotinabhängigkeit erläutert (Raupach et al. 2010). Ferner wurden die positiven Effekte einer Tabakentwöhnung auf die Morbidität und Mortalität bei KHK-Patienten dargeboten. Den Mitarbeitern sollte auf diese Weise die individuelle gesundheitliche Relevanz eines Rauchstopps für die Patienten verdeutlicht werden.

Nachfolgend wurde der Studienaufbau erklärt und die Standard Operating Procedures (SOPs) mit den einzelnen jeweils von den ärztlichen und pflegerischen Mitarbeitern erforderlichen Tätigkeiten vorgestellt. Die in Form einer Tabelle dargestellten zusätzlich anfallenden Aufgaben wurden auch hinsichtlich ihrer Integration in den Arbeitsalltag mit den Mitarbeitern diskutiert.

Weiterer Inhalt war die Erläuterung des 5 A-Schemas (Fiore et al. 2008). Dieses fungierte als eine Art Leitlinie und sollte den Schulungsteilnehmern als Orientierungshilfe für die Ausstiegsberatung der rauchenden Patienten dienen. Es folgten Informationen zur Nikotinersatztherapie sowie eine Anleitung zur Handhabung der verfügbaren Präparate. 
Nach einer 10-minütigen Pause folgte der zweite Abschnitt der Schulung. In diesem Teil ging es um die praktische Umsetzung der Ausstiegsberatung. Unter der Moderation von Herrn Lindinger wurden gemeinsam mit den Teilnehmern Anregungen und Strategien für die Gesprächsführung gesammelt und konkrete Formulierungen diskutiert, die als roter Faden nützlich sein könnten. Es ging um den persönlichen Zugang zu den Patienten, die konkrete Thematisierung des Rauchens im Rahmen eines Aufnahmegesprächs und einen adäquaten Ratschlag zum Rauchverzicht. In die Diskussion flossen sowohl die psychologisch und verhaltenstherapeutisch fundierten Ansätze der Tabakentwöhnungsberatung als auch die individuellen Meinungen und Erfahrungen der Teilnehmer ein. Besonderes Augenmerk wurde auf die Authentizität gegenüber den Patienten gelegt.

Abschließend wurden eventuell auftretende Schwierigkeiten thematisiert, beispielsweise wie man sich verhalten solle, wenn ein Patient den Mitarbeiter auf dessen eigenes Rauchverhalten aufmerksam machen würde.

\subsection{Erhebungsinstrumente}

Die Erhebung der Daten der hospitalisierten rauchenden Patienten ebenso wie die Befragung der auf den Stationen eingesetzten Mitarbeiter erfolgte mit Hilfe von Fragebogen. Diese wurden vor Studienbeginn in Zusammenarbeit mit Dipl.-Psych. Peter Lindinger und Christa Rustler vom Deutschen Netz Rauchfreier Krankenhäuser und Gesundheitseinrichtungen (DNRfK) entwickelt. Alle im Folgenden beschriebenen Erhebungsinstrumente sind im Anhang dieser Arbeit zu finden.

\subsubsection{Patientenfragebogen}

\subsubsection{Fragebogen vor Intervention}

Mit Hilfe des Fragebogens vor Intervention (im Anhang als Erhebungsbogen Patienten Aufnahme) wurden zunächst die Basisdaten Geschlecht, Alter und Anzahl der Raucherjahre erhoben. Der durchschnittliche Tabakkonsum pro Tag musste angegeben werden für Zigaretten, Zigarren bzw. Zigarillos, Pfeifen und elektrische Zigaretten sowie für Wasserpfeifen pro Woche. Ferner sollte angegeben werden, ob dem Studienteilnehmer bekannt sei, dass das Uniklinikum Göttingen ein rauchfreies Krankenhaus ist. Die Frage nach einem eventuellen Aufhörversuch in der Vergangenheit konnte ebenfalls mit „Ja“ oder „Nein“ beantwortet wer- 
den. Traf Ersteres zu, so sollte in einer Freitextzeile der Anlass zu diesem Aufhörversuch genannt werden.

Die beiden nächstfolgend aufgeführten Punkte wurden sowohl im Aufnahmefragebogen als auch im Fragebogen nach Intervention erhoben. Zum einen ging es um die individuelle Rauchstoppmotivation, zum anderen um die Sicherheit des Patienten, für eine Dauer von mindestens vier Wochen mit dem Rauchen aufhören zu können. Beide sollten jeweils mit Hilfe einer sechsstufigen Skala beantwortet werden, die mit ihren entsprechenden Extremen „sehr motiviert“ bzw. „sehr sicher“ sowie ,überhaupt nicht motiviert“ bzw. „,̈berhaupt nicht sicher" dargestellt war.

Weiterhin im Fragebogen vor Intervention thematisiert wurden supportive Maßnahmen bei der Tabakentwöhnung wie Beratung, Medikamente, Informationen diesbezüglich oder die Anbindung an die Tabakentwöhnungsambulanz. Wie hilfreich diese im Rahmen des jetzigen Klinikaufenthaltes eingeschätzt wurden, konnte durch Ankreuzen eines der Felder „sehr hilfreich“, „hilfreich“, „wenig hilfreich“ oder „gar nicht hilfreich, allein meine eigene Willenskraft ist entscheidend“" angegeben werden. Bei der letzten Frage auf der Vorderseite des Aufnahmefragebogens sollte der Studienteilnehmer angeben, ob er während des Klinikaufenthaltes von Ärztinnen oder Ärzten bezüglich einer Raucherentwöhnung beraten werden möchte. Zur Auswahl standen „Ja“, „Nein“ und „Weiß nicht“.

Auf der Rückseite wurde die Aufnahmeerhebung komplettiert durch die sechs Fragen des Fagerström-Tests für Nikotinabhängigkeit (FTND) (Heatherton et al. 1991).

\subsubsection{Fragebogen nach Intervention}

Der Fragebogen nach Intervention (im Anhang als Erhebungsbogen Patienten - Entlassung) umfasste die folgenden Punkte:

Zu Beginn war anzugeben, ob während des stationären Aufenthaltes eine Tabakentwöhnungsberatung erfolgte, wobei bei einer positiv ausfallenden Antwort differenziert werden musste zwischen einer pflegerischen oder ärztlichen Intervention. Diese konnte zudem unter Angabe von Gründen positiv oder negativ bewertet werden.

Der nächste Abschnitt zielte ebenfalls auf die Zufriedenheit des Patienten mit der Beratung, sofern diese stattfand. Fünf Aussagen waren aufgeführt mit einer jeweils dazugehörigen sechsstufigen Skala, die es anzukreuzen galt. Die beiden Extreme der Skala lauteten „trifft voll zu“ sowie „trifft nicht zu“. Inhaltlich thematisierten die Aussagen den durch die Mitarbeiter kommunizierten Motivations-Anstoß, mit dem Rauchen aufzuhören, das Gefühl, hinsicht- 
lich des Rauchens gut aufgeklärt worden zu sein, eine eventuell empfundene Belästigung durch die Ratschläge zum Rauchverzicht, einen zusätzlichen Informationsbedarf sowie die Einschätzung, wie wichtig der Rauchverzicht für die individuelle Genesung sei.

Erfolgte während des stationären Aufenthaltes keine Intervention, so entfielen die letztgenannten Punkte. Ob während des Klinikaufenthaltes eine Reduktion des Tabakkonsums oder gar ein Rauchverzicht unternommen wurde, sollte in der nächsten Frage beantwortet werden. Bei Ankreuzen von „Ja“ waren weitere Angaben zu machen. In zwei Freitextzeilen sollte zum einen der wichtigste Grund dafür aufgeführt werden, zum anderen sollte genannt werden, was dabei besonders hilfreich war. Ferner mussten eventuell genutzte Hilfsmittel angekreuzt werden, wobei eine Mehrfachauswahl möglich war. Als Ankreuzmöglichkeiten waren aufgeführt: „keine“, „Broschüre“, „Telefonberatung“, „Nikotinersatzmittel“, „“ein Termin in der Entwöhnungsambulanz“ sowie ,andere“ unter Angabe dieser.

Eine bereits während des stationären Aufenthaltes erfolgte Anbindung an die klinikeigene Tabakentwöhnungsambulanz oder die Absicht, in den kommenden vier Wochen einen dort angebotenen Kurs wahrzunehmen, wurde durch die nächsten beiden Punkte erhoben.

Bei der letzten Frage des Entlassungsfragebogens konnten die Studienteilnehmer angeben, inwieweit sie die Umsetzung der Initiative „rauchfreies Krankenhaus“ an der Uniklinik Göttingen für konsequent empfanden. Zur Auswahl standen „Ja“, „Nein“ und „keine Meinung“ mit der Möglichkeit, in einer Freitextzeile Anmerkungen diesbezüglich zu machen.

\subsubsection{Mitarbeiterfragebogen}

\subsubsection{Erhebungsbogen Pflege vor Intervention}

In der Eingangsfrage des Erhebungsbogen Pflege vor Intervention (Fragebogen im Anhang) sollte angegeben werden, wie wichtig es für die Mitarbeiter sei, den Rauchstatus des Patienten zu kennen. Angekreuzt werden konnte eine sechsstufige Skala, welche von ,sehr wichtig“ bis „gar nicht wichtig“ reichte.

Mit Hilfe der nächsten Frage wurde erhoben, inwieweit die Kenntnis des Rauchstatus einen Einfluss auf die Stationsarbeit oder den Umgang mit dem Patienten hat. Gab es einen solchen Einfluss, so sollte dieser nach Ankreuzen des Feldes „Ja“ in einer Freitextzeile spezifiziert werden, andernfalls war das Feld „Nein“ anzukreuzen.

Weiter unten im Bogen wurde ermittelt, ob rauchende Patienten bereits während des Klinikaufenthaltes bezüglich einer Tabakentwöhnung beraten werden sollten, ebenfalls anhand einer sechsstufigen Skala. 
Die nachstehend dargestellten Punkte waren in beiden Fragebogen, also auch im Erhebungsbogen Pflege nach Intervention aufgeführt:

So sollte zum einen von den Mitarbeitern genannt werden, wie viele Patienten (75 - $100 \%, 50$ - $75 \%, 25$ - $50 \%, 5$ - $25 \%$, weniger als $5 \%$ ) von ihnen persönlich hinsichtlich einer Tabakentwöhnung beraten werden. Ebenso, wie kompetent sie sich bei der Beratung der Raucher fühlen. Zum Ankreuzen standen hierfür sechs Felder zur Verfügung, von „sehr kompetent“ bis ,gar nicht kompetent" reichend.

Der anschließende Abschnitt thematisierte das Wissen und die Fähigkeiten der Mitarbeiter in Bezug auf die Entwöhnungsberatung. Genannt werden sollte im Rahmen einer Selbsteinschätzung, inwieweit Kenntnisse über die Grundlagen der Tabakabhängigkeit, Kenntnisse über die Folgeerkrankungen des Tabakkonsums, Wissen über verhaltensbezogene und medikamentöse Maßnahmen sowie die Fähigkeit, Patienten zum Rauchverzicht zu motivieren, vorliegen. Zur Beantwortung jeder dieser vier Aussagen waren sechsstufige Skalen aufgeführt mit den Extremen „trifft voll zu“ beziehungsweise „trifft nicht zu“.

In einem weiteren Punkt sollten die Mitarbeiter einschätzen, für wie groß sie den Effekt der von ihnen durchgeführten Raucherentwöhnungsberatung für die individuelle Genesung und Gesundheitsförderung rauchender Patienten halten. Die dazugehörige Skala war sechsstufig und das Spektrum reichte von ,einen großen Effekt" bis ,gar keinen Effekt““

Ferner war anzugeben, wie hoch der zeitliche Aufwand (0 - $5 \mathrm{~min}, 5$ - $10 \mathrm{~min}, 10$ - $20 \mathrm{~min}$, > 20 min) pro Beratung ausfiel. Die abschließende Frage zielte auf das persönliche Rauchverhalten der Mitarbeiter. Hier wurde differenziert zwischen Nichtrauchern ohne früheren Tabakkonsum, Nichtrauchern nach früherem Tabakkonsum oder aktiven Rauchern.

\subsubsection{Erhebungsbogen Pflege nach Intervention}

Der für Phase II konzipierte Erhebungsbogen Pflege nach Intervention beinhaltete neben den letzten, oben genannten Punkten, noch die folgenden:

Eingangs wurde nach der Teilnahme an der Trainingsmaßnahme gefragt. Hatte diese stattgefunden, so konnte neben der Vergabe einer Schulnote dafür (Note 1 - 6) in zwei separaten Freitextzeilen jeweils positive und negative Kritik geäußert werden. Darüber hinaus sollte durch Ankreuzen einer sechsstufigen Skala angegeben werden, für wie sinnvoll es gehalten wird, zur Festigung der durch diese Trainingsmaßnahme vermittelten Kenntnisse und Fähigkeiten eine erneute Schulung durchzuführen. 
In einem weiteren Absatz des Erhebungsbogen waren in Form von fünf Aussagen potenzielle Reaktionen der Patienten im Rahmen einer durchgeführten Entwöhnungsberatung aufgelistet, die dahingehend eingestuft werden sollten, wie häufig sie dem beratenden Mitarbeiter entgegnet wurden. Zur Einstufung standen jeweils sechsstufige Skalen zur Verfügung. Als Reaktionen wurden Interesse, der Wunsch nach zusätzlicher Information oder Beratung, eine vorhandene Bereitschaft zum Rauchstopp, einen tatsächlich durchgeführten Rauchstopp sowie eine Ablehnung der Beratung angeführt. Zusätzlich konnten in einer freien Zeile sonstige beobachtete Reaktionen formuliert werden.

Ein in analoger Weise aufgebauter Absatz thematisierte die durch Einführung der SOP für die Mitarbeiter zusätzlich anfallende Arbeit. Ebenfalls anhand sechsstufiger Skalen sollte angegeben werden, inwieweit sich die zusätzliche Arbeit in den normalen Arbeitsalltag integrieren ließ, inwiefern der Mitarbeiter im Laufe der Zeit routinierter wurde und die Integration der SOP-spezifischen Aufgaben deshalb besser möglich war, ob eine weitere Betreuung rauchender Patienten auch über die Projektlaufzeit hinaus denkbar sei, ob diese Maßnahmen auch auf anderen Stationen eingeführt werden sollten und inwieweit sich der Mitarbeiter vorstellen könnte, auch andere Kollegen zur Entwöhnungsberatung zu motivieren.

Abschließend wurde den Mitarbeitern Raum gelassen für eventuelle Bemerkungen oder Anregungen.

\subsubsection{Leitfaden für die Mitarbeiter-Interviews}

Vor Beginn der Interviews erfolgte zunächst eine kurze Einleitung, in der sich die Interviewerin vorstellte und ihre Unabhängigkeit von der Raucherentwöhnung im Allgemeinen sowie insbesondere von der Entwicklung und Implementierung der SOP betonte. Die interviewte Person wurde ermutigt, sämtliche Erfahrungen und persönliche Ansichten in Bezug auf die SOP, die Schulungsmaßnahme und die Beratung rauchender Patienten zu äußern. Erklärtes Ziel des Interviews sei die Überarbeitung und Optimierung der SOP sowie der Schulungsmaßnahme. Die gewonnenen Erkenntnisse wurden ausschließlich für diesen Zweck verwendet. Ferner wurden dem Probanden der generelle Ablauf und die Modalitäten des Interviews ebenso wie die weitere Handhabung der Daten erläutert.

Im Folgenden wird der Leitfaden für das Interview dargestellt. Dieser Fragenkatalog diente dem Leiter des Interviews als roter Faden. Die Fragen waren offen formuliert und deckten 
sämtliche zu untersuchenden Aspekte ab. Die exakte Reihenfolge der Fragen war dabei nicht streng festgelegt sofern alle relevanten Punkte erhoben wurden.

Die Leitfäden für die Einzelinterviews mit den ärztlichen Mitarbeitern differierten geringfügig von denen der pflegerischen Mitarbeiter. Dies wird an den entsprechenden Stellen erläutert, außerdem sind die Dokumente dem Anhang dieser Arbeit beigefügt.

$\mathrm{Zu}$ Beginn des Interviews wurde die individuelle Ansicht und Meinung über die eingeführte SOP zur Betreuung rauchender Patienten erfragt, auch hinsichtlich der persönlich empfundenen Notwendigkeit und des Nutzens dieser Standard Operating Procedures.

Im Rahmen der SOP fielen für die jeweiligen Mitarbeiter zusätzliche Aufgaben an. Für das pflegende Personal beispielsweise die Dokumentation des Rauchstatus, die Kennzeichnung der Raucher an der Wandtafel, die Erläuterung der Handhabung der Medikamente oder die Anbindung an die klinikeigene Tabakentwöhnungsambulanz. Ärztlicherseits fielen Kurzberatungen zum Rauchstopp an sowie die Verschreibung von Medikamenten zur Unterstützung dessen und die anschließend korrekte Dokumentation der Interventionen in den Arztbriefen unter einheitlicher Nomenklatur. Die Mitarbeiter sollten äußern, wie sie darüber denken und ihre Meinung zur Aufgabenverteilung bzw. zu ihrer Rolle innerhalb der Betreuung der hospitalisierten Raucher darlegen. Negative Aspekte und Verbesserungsvorschläge sollten gegebenenfalls genannt werden.

Es folgten weitere Fragen nach den Veränderungen, die von dem Mitarbeiter seit Einführung der SOP vernommen wurden, außerdem welche Auswirkungen die SOP auf den persönlichen Arbeitsalltag hatte und wie sie im Einzelnen umgesetzt wurde. Es sollten Erfahrungen und Empfindungen geäußert werden, die im Zusammenhang mit dem Beratungsgespräch mit dem Raucher erlebt wurden. Ferner wurde die Meinung bezüglich der medikamentösen Therapie thematisiert, auch in Bezug auf die Kompetenz, ihre Anwendung zu erläutern.

Als Einführung und gleichermaßen als Vorbereitung auf die SOP wurde eine Trainingsmaßnahme angeboten. Dem ärztlichen Personal wurde im Vorhinein per E-Mail Informationen bezüglich des Projektes und der SOP übermittelt. Den Mitarbeitern wurde im Interview jeweils die Möglichkeit gegeben, Meinung und Kritik über diese Vorbereitungsmaßnahmen zu nennen. Auftretende Schwierigkeiten bei der Umsetzung der SOP sowie die Beurteilung der eigenen Fähigkeit, das Thema „Rauchen“ mit dem Patienten zu besprechen, sollten geäußert werden. In Bezug auf die Schulungsmaßnahme wurde nach Verbesserungsvorschlägen gefragt sowie danach, ob ein erneut stattfindendes Training für hilfreich gehalten würde. 
Der abschließende Punkt der Einzelinterviews zielte auf den persönlich empfundenen Stellenwert, den die befragten Mitarbeiter der Behandlung rauchender Patienten an der Universitätsklinik Göttingen beimessen.

\subsection{Statistische Analyse}

Primärer Endpunkt der vorliegenden prospektiven Interventionsstudie war die Häufigkeit von Tabakentwöhnungsinterventionen bei hospitalisierten Rauchern während des Klinikaufenthaltes. In Anlehnung an Daten früherer Erhebungen an der UMG (Raupach et al. 2011) wurde eine Interventionshäufigkeit von $20 \%$ in Phase I angenommen mit der Erwartung, eine durch die studienbezogenen Maßnahmen induzierte Steigerung dieser um $15 \%$ auf einem $\alpha$-Level von $5 \%$ zu beobachten. Um einen solchen Unterschied mit einer Power von $80 \%$ zu detektieren, mussten für jede der beiden Studienphasen jeweils 48 Studienteilnehmer rekrutiert werden.

Die in den Erhebungsbogen eingesetzten sechsstufigen Skalen wurden in dichotome Variablen transformiert, wobei die beiden positiven Optionen der Bewertungsskala als bestätigende Haltung gewertet wurden; die übrigen vier Optionen (neutral / negativ) wurden als nichtbestätigende Haltung zusammengefasst.

Die generierten Daten wurden mit Hilfe des Statistikprogramms SPSS $\odot$ (Version 14.0) analysiert. Kontinuierliche Variablen wurden dargestellt als Mittelwert \pm Standardabweichung.

Zur Untersuchung statistischer Gruppen-Unterschiede wurden bei metrischen Variablen tTests angewendet, bei dichotomen Variablen kamen $\chi^{2}$-Tests zum Einsatz.

Die akustisch-elektronisch aufgezeichneten Einzelinterviews wurden im Anschluss wörtlich transkribiert. Die Transkripte stellten die Grundlage für die nachfolgende Analyse dar, welche nach dem Prinzip der Template Analysis (King 2004) erfolgte und an dieser Stelle kurz erläutert wird:

Die Template Analysis als qualitative Methode zur Textanalyse ermöglicht es, die wesentlichen Inhalte aus umfangreichen und unstrukturierten Texte (z.B. Abschriften von Interviews) zu extrahieren und übersichtlich darzustellen. Zentrales Element ist dabei die Formulierung 
sogenannter Themen (themes) zur Kategorisierung der Texte, welche im Verlauf der Auswertung identifiziert und ausgearbeitet werden müssen.

Entsprechend wurden wichtige Aussagen in den Äußerungen der interviewten Mitarbeiter hinsichtlich ihres Inhalts bestimmten Themen zugeordnet. Dieser Prozess wird als Kodierung (coding) bezeichnet. In einem iterativen Prozess wird die Struktur der extrahierten Daten im Laufe der Analyse modifiziert und optimiert. Häufig erweisen sich dabei einige, zum Teil im Vorhinein (a priori) in Erwartung an gewisse Ergebnisse formulierte Themen nach der Auswertung der Transkripte als unbrauchbar für die Einteilung der Textaussagen, während andere Themen neu aufkommen.

Die auf diese Weise herausgearbeiteten Themen wurden dann in Bezug auf die drei Aspekte des „COM-B“- Modells (siehe Kapitel 1.7), also die Verhaltensdeterminanten Fertigkeiten (capability), Gelegenheit (opportunity) und Motivation (motivation) eingeordnet.

Gemäß des Votums der Ethik-Kommission der Medizinischen Fakultät der Universität Göttingen vom 16.01.2011 bestanden keine ethischen und rechtlichen Bedenken gegen die Durchführung des vorliegenden Forschungsvorhabens (Antragsnummer: 11/10/10). 


\section{Ergebnisse}

\subsection{Probandencharakteristika}

In Phase I der Studie, also vor Implementierung der SOP, konnten insgesamt 78 Raucher identifiziert werden. Davon mussten drei Patienten aufgrund mangelnder Deutschkenntnisse, nicht-invasiver Beatmung per Mund-Nasen-Maske sowie fehlender Einwilligung von der Studienteilnahme ausgeschlossen werden.

In Phase II wurden zusammen 79 Raucher ermittelt, wobei vier von der Teilnahme ausgenommen wurden. Die Gründe dafür waren fehlende Zustimmung zur Teilnahme (3 Patienten) sowie nicht-invasive Beatmung (ein Patient). Somit konnten für beide Phasen der Studie jeweils 75 Raucher rekrutiert werden.

Inwieweit die Patientenkollektive der beiden Studienabschnitte innerhalb der erhobenen Basisdaten und Merkmale differierten, ist in Tab. 4 übersichtsweise dargestellt.

Die von den Patienten gemachten Angaben bezüglich der Anlässe für den letzten Rauchverzicht beziehen sich dabei auf die Subgruppe derjenigen Raucher, die bereits einen Aufhörversuch in der Vergangenheit unternommen hatten. Dies waren in Phase I insgesamt 53 von 75 (70,7\%), in Phase II 51 von 75 (68\%) Raucher.

Als Hauptdiagnose bei Aufnahme war sowohl in Phase I als auch in Phase II das akute Koronarsyndrom mit 45,3\% bzw. 30,7 \% am häufigsten zu verzeichnen. $\mathrm{Zu}$ den weiteren Hauptaufnahmediagnosen der Studienteilnehmer siehe ebenfalls Tab. 4. 
Tab. 4: Patientencharakteristika, Rauchverhalten und Hauptaufnahmediagnosen angegeben in Prozent (absolute Zahlen) oder als Mittelwert \pm Standardabweichung

\begin{tabular}{|c|c|c|c|}
\hline & $\begin{array}{l}\text { Phase I } \\
(\mathrm{n}=75)\end{array}$ & $\begin{array}{l}\text { Phase II } \\
(\mathrm{n}=75)\end{array}$ & $p-$ Wert \\
\hline \multicolumn{4}{|c|}{ Patientencharakteristika } \\
\hline Geschlecht (Anteil männlicher Raucher) & $66,7(50)$ & $69,3(52)$ & 0,726 \\
\hline Alter (Jahre) & $55,9 \pm 12.8$ & $55,9 \pm 11,3$ & 0,973 \\
\hline Raucherjahre & $34,4 \pm 12,5$ & $35,9 \pm 11,9$ & 0,471 \\
\hline Zigaretten pro Tag & $20,1 \pm 9,9$ & $18,5 \pm 9,6$ & 0,305 \\
\hline Packungsjahre, $p y$ & $36,3 \pm 23,8$ & $35,3 \pm 22,5$ & 0,811 \\
\hline Anteil der Zigarettenraucher & $97,3(73)$ & $97,3(73)$ & 1 \\
\hline FTND & $5,0 \pm 2,2$ & $3,7 \pm 2,1$ & $<0,001$ \\
\hline $\begin{array}{l}\text { Anteil der Patienten mit einem Aufhörversuch in } \\
\text { der Vergangenheit }\end{array}$ & $70,7(53)$ & $68,0(51)$ & 0,723 \\
\hline \multicolumn{4}{|c|}{$\begin{array}{c}\text { Anlass für den letzten Aufhörversuch } \\
\text { (bezogen auf die Subgruppe mit einem Aufhörversuch in der Vergangenheit) }\end{array}$} \\
\hline Gesundheit / Krankheit & $45,3(24)$ & $52,9(27)$ & \\
\hline Abnehmendes Verlangen nach dem Rauchen & $26,4(14)$ & $15,7(8)$ & \\
\hline Finanzielle Aspekte & $1,9(1)$ & $7,8(4)$ & \\
\hline Ärztlicher Rat & $1,9(1)$ & $2,0(1)$ & \\
\hline Sonstiges & $18,9(10)$ & $19,6(10)$ & \\
\hline Keine Angabe & $5,7(3)$ & $2,0(1)$ & \\
\hline \multicolumn{4}{|c|}{ Hauptdiagnosen bei Aufnahme } \\
\hline Akutes Koronarsyndrom (STEMI / NSTEMI) & $45,3(34)$ & $30,7(23)$ & \\
\hline Koronare Herzerkrankung & $12,0(9)$ & $13,3(10)$ & \\
\hline Herzinsuffizienz und Kardiomyopathien & $10,7(8)$ & $10,7(8)$ & \\
\hline Arrhythmien & $6,7(5)$ & $20,0(15)$ & \\
\hline Pneumonie & $4,0(3)$ & $1,3(1)$ & \\
\hline COPD & $5,3(4)$ & $4,0(3)$ & \\
\hline Herzklappenfehler & $5,3(4)$ & $1,3(1)$ & \\
\hline Andere & $10,7(8)$ & $18,7(14)$ & \\
\hline
\end{tabular}




\subsection{Ergebnisse der Patientenbefragung}

Die weitere Ergebnisdarstellung der Befragung der rauchenden Patienten erfolgt in Anlehnung an die in Kapitel 1.8 formulierten Aspekte. Ein Auszug der wesentlichen Resultate dieser Erhebung liefert Tab. 5.

\section{Aspekt 1: Interventionshäufigkeit bei Rauchern durch ärztliche und pflegerische Mitar- beiter/innen}

Im ersten Studienabschnitt (Phase I) gaben fünf der 75 Patienten an, während ihres stationären Aufenthaltes von einer Ärztin oder einem Arzt hinsichtlich einer Tabakentwöhnung beraten worden zu sein. Im zweiten Studienabschnitt (Phase II) waren dies 29 Patienten $(6,7 \%$ vs. $38,7 \% ; \mathrm{p}<0,001)$.

Eine Entwöhnungsberatung durch die pflegerischen Mitarbeiter wurde in Phase I von einem Raucher angegeben, in Phase II waren es zwei Raucher. Dieser Unterschied war nicht signifikant $(\mathrm{p}=0,560)$.

Eine Beratung sowohl durch ärztliche als auch durch pflegerische Mitarbeiter wurde von keinem Patienten in Phase I und von einem Patienten in Phase II berichtet.

Tab. 5: Interventionshäufigkeit, Aufhörversuch mit eventueller Inanspruchnahme von Hilfe und Anbindung an die Tabakentwöhnungsambulanz angegeben in Prozent (absolute Zahlen)

\begin{tabular}{|l|c|c|c|}
\hline \multicolumn{3}{|c|}{ Angaben der Raucher/innen im Rahmen der Patientenbefragung } \\
\hline $\begin{array}{l}\text { Phase I } \\
(\mathrm{n}=75)\end{array}$ & $\begin{array}{c}\text { Phase II } \\
(\mathrm{n}=75)\end{array}$ & p-Wert \\
\hline $\begin{array}{l}\text { Entwöhnungsberatung durch einen ärztlichen } \\
\text { Mitarbeiter }\end{array}$ & $6,7(5)$ & $38,7(29)$ & $<0,001$ \\
\hline $\begin{array}{l}\text { Entwöhnungsberatung durch einen pflegerischen } \\
\text { Mitarbeiter }\end{array}$ & $1,3(1)$ & $2,7(2)$ & 0,56 \\
\hline Aufhörversuch während des Klinikaufenthaltes & $94,7(71)$ & $100(75)$ & 0,043 \\
\hline Aufhörversuch ohne Hilfe (z.B. Medikamente) & $94,7(71)$ & $90,7(68)$ & 0,008 \\
\hline $\begin{array}{l}\text { Anbindung an die klinikinterne Entwöhnungs- } \\
\text { ambulanz }\end{array}$ & $0(0)$ & $2,7(2)$ & 0,152 \\
\hline
\end{tabular}




\section{Aspekt 2: Zufriedenheit der Patienten mit der Entwöhnungsberatung}

Von insgesamt sechs durchgeführten Entwöhnungsberatungen in Phase I bewerteten fünf Raucher das Beratungsgespräch positiv. Die Bewertung einer erfolgten Entwöhnungsberatung konnte unter Angabe von Gründen in der entsprechenden Freitextzeile für „gute“ oder „schlechte Beratung“ vorgenommen werden.

Als Begründung wurde von den meisten eine gute Aufklärung und Vergegenwärtigung der Risiken eines fortgesetzten Tabakkonsums genannt. Ein weiterer Raucher führte seine Zufriedenheit mit der Beratung vor allem auf die allgemeine Arztrolle bzw. den Status des Arztes zurück (Zitat: „Eine Beratung von einem Arzt ist immer gut").

Ein von ärztlicher Seite beratener Patient erteilte eine negative Beurteilung, da er ,,außer einem Fragebogen nichts Konkretes in der Hand halten“ würde.

Im zweiten Studienabschnitt erfolgten 31 Beratungsgespräche zum Rauchstopp. 26 Raucher empfanden dieses positiv, wohingegen ein Patient negative Kritik äußerte mit dem Hinweis, er fühle sich „durch ein solches Gespräch zu sehr unter Druck gesetzt““.

Vier Patienten bewog das Angebot von Hilfe zu einer guten Bewertung, zehn Raucher gaben als Begründung eine „informative“ und „aufklärende“ Darstellung des Themas Rauchen einschließlich der Entwöhnungsmöglichkeiten an. Manche würdigten in diesem Zusammenhang auch das Auftreten und die Gesprächsführung des ärztlichen Mitarbeiters, welches unter anderem als „engagiert“, „empathisch“, „glaubwürdig“ und „nicht aufdringlich“ bezeichnet wurde. Für einige war auch hier der Status bzw. die Rolle des Arztes ausschlaggebend. So bemerkte ein Patient: „Wenn ein Arzt das Rauchen nicht anspricht, dann kann es ja nicht so schlimm sein“.

Einige andere Patienten begründeten ihre Zufriedenheit damit, dass sie durch die Beratung die gesundheitliche Relevanz einer Abstinenz erkannten (Zitat: „Mir wurden die Augen geöffnet").

\section{Aspekt 3: Dokumentation des Rauchstatus sowie einer Entwöhnungs-Intervention}

In Phase I wurde bei 66 von 75 Rauchern (88 \%) ein entsprechender Vermerk über den Rauchstatus im Aufnahmebogen der Station vorgenommen. Im Entlassungsbrief wurde das Rauchen bei insgesamt 63 Patienten (84 \%) mit unter den Diagnosen aufgeführt. 
Im zweiten Studienabschnitt (Phase II) war der Rauchstatus bei 65 Patienten (86,7 \%) im Aufnahmebogen vermerkt, während die Diagnose Rauchen bei 56 Patienten $(74,7 \%)$ im Arztbrief dokumentiert wurde.

Eine Dokumentation entsprechend durchgeführter Entwöhnungsinterventionen im Entlassungsbrief wurde in Phase I in keinem und in Phase II in sieben Fällen vorgenommen (p = 0,007). Ähnlich verhielt es sich bei der Dokumentation der Verordnung von Entwöhnungsmedikamenten (0 Fälle in Phase I vs. 5 Fälle in Phase II; p =0,023) sowie dem Verweis auf eine erfolgte Anbindung an die klinikeigene Entwöhnungsambulanz (0 Fälle in Phase I vs. 6 Fälle in Phase II; $p=0,012$ ).

\section{Aspekt 4: Bereitschaft der betroffenen Patienten, das Rauchen aufzugeben}

Die Anzahl der Studienteilnehmer in Phase I, die eine hohe Bereitschaft aufwiesen, mit dem Tabakrauchen aufzuhören, erhöhte sich im Laufe des stationären Aufenthaltes von 43 auf 44. Eine hohe Bereitschaft wurde dann angenommen, wenn in der sechsstufigen Skala der entsprechenden Frage eine der beiden Optionen am positiven Pol der Bewertungsskala angekreuzt wurde.

In der nach der Intervention liegenden zweiten Studienphase stieg die Zahl der zum Rauchverzicht stark motivierten Patienten zwischen der Klinikaufnahme und der Entlassung von 45 auf 51.

In Bezug auf die Sicherheit, für mindestens vier Wochen mit dem Rauchen aufhören zu können, wählten zu Beginn des Klinikaufenthaltes 39 Raucher aus Phase I eine der beiden positiven Skalenoptionen. Bei Entlassung kamen 42 Raucher zu dieser Einschätzung.

In Phase II erklärten bei Aufnahme 40 Patienten eine hohe Sicherheit zum mindestens vierwöchigen Rauchverzicht, bei Entlassung insgesamt 47.

Weiterhin wurde ermittelt, wie vielen Studienteilnehmern es im Verlaufe des stationären Aufenthaltes gelang, eine Reduktion des gewohnten Tabakkonsums vorzunehmen oder gar eine völlige Rauchabstinenz zu erreichen. Dies traf für 71 von 75 Rauchern in Phase I sowie für alle rauchenden Patienten in Phase II zu. 
Dabei nahm keiner der 71 Patienten aus Phase I ein Hilfsmittel in Anspruch. In der zweiten Phase griffen fünf Raucher auf eine Nikotinersatztherapie sowie zwei Raucher auf eine Informationsbroschüre als unterstützende Maßnahme zurück.

In Tab. 6 sind die von den Patienten angegebenen ausschlaggebenden Gründe für den vorgenommenen Entwöhnungsversuch während des Klinikaufenthaltes dargestellt.

Tab. 6: Wichtigster Grund für einen Entwöhnungsversuch im Klinikum. Angaben in Prozent (absolute Zahlen)

\begin{tabular}{|l|c|c|}
\hline & vor SOP (n = 71) & nach SOP (n = 75) \\
\hline Gesundheit / Krankheit & $47,9(34)$ & $33,3(25)$ \\
\hline keine Lust mehr / mal probieren / Wille & $21,1(15)$ & $36,0(27)$ \\
\hline Bettlägerigkeit / Immobilität & $14,1(10)$ & $9,3(7)$ \\
\hline Krankenhausaufenthalt & $4,2(3)$ & $0(0)$ \\
\hline Umstände / Rauchverbot & $7,0(5)$ & $18,7(14)$ \\
\hline Sonstige (Angst vor Tod) & $4,2(3)$ & $2,7(2)$ \\
\hline keine Angabe & $1,4(1)$ & $0(0)$ \\
\hline
\end{tabular}

\section{Aspekt 5: Zahl der Raucher, welche das Angebot der klinikeigenen Tabakentwöh- nungsambulanz in Anspruch nahmen}

Keiner der 75 teilnehmenden Raucher aus Phase I hatte während des stationären Aufenthaltes einen Termin in der klinikeigenen Tabakentwöhnungsambulanz. In Phase II stellten sich zwei der 75 Raucher dort vor $(\mathrm{p}=0,152)$.

Die Frage, ob der Studienteilnehmer innerhalb der nächsten vier Wochen einen Entwöhnungskurs in der Ambulanz wahrnehmen möchte, wurde in beiden Studienabschnitten von jeweils neun Rauchern (12\%) mit „Ja“ beantwortet.

\section{Aspekt 6: Die zusätzliche Arbeitsbelastung der Mitarbeiter}

Dieser Punkt wird im nachfolgenden Kapitel im Rahmen der Ergebnisdarstellung der Mitarbeiterbefragung abgehandelt. 


\subsection{Ergebnisse der Mitarbeiterbefragung}

Auf den drei im Rahmen der Studie involvierten kardiologischen Stationen nahmen insgesamt 22 pflegerische Mitarbeiter an beiden Fragebogenerhebungen (Phase I und Phase II) teil.

Unter den ärztlich tätigen Mitarbeitern fiel die Stichprobe geringer aus. Bis zum Ende der Erhebungsperiode konnten lediglich fünf komplette Datensätze generiert werden, so dass eine aussagekräftige quantitative Analyse der Daten nicht möglich war. Aufschluss über Erfahrungen und Eindrücke während der Projektlaufzeit sowie die Beurteilung der Umsetzbarkeit und Integration der SOP durch die auf den Stationen eingesetzten Ärzte sollen die durchgeführten Mitarbeiter-Interviews liefern.

Bei der Darstellung der folgenden Ergebnisse handelt es sich um die Auswertung der Fragebogen-Erhebung unter den Pflegekräften.

Von den 22 Pflegerinnen und Pflegern nahmen insgesamt 12 an der zwischen Phase I und Phase II angesetzten strukturierten Trainingsmaßnahme der Studie teil, was einer Teilnahmequote von $54,5 \%$ entspricht.

Mit einem fünfstufigen Item wurde erhoben, welchen Anteil der rauchenden Patienten die Pflegekräfte durchschnittlich beraten. Es fällt auf (siehe Tab. 7), dass vor der Intervention vier von fünf Pflegekräften angaben, fast keine Beratungen ( $<5 \%$ der Patienten) durchzuführen. Dieser Anteil hatte sich nach der Intervention auf $60 \%$ reduziert, obwohl nur jede zweite Pflegekraft am Training teilgenommen hatte. Zugleich war der Anteil der Pflegekräfte, die bis zu einem Viertel ihrer rauchenden Patienten hinsichtlich einer Entwöhnung berieten, in Phase II mit 36,4 \% deutlich höher als in Phase I (13,6 \%).

Der Trend zu mehr Beratungen war dabei nicht von einer Verlängerung der einzelnen Gespräche begleitet. So gab die Hälfte der Befragten in Phase II an, dass das Patientengespräch zum Thema „Rauchen“ im Schnitt maximal fünf Minuten dauere; ein weiteres Drittel erklärte, pro Gespräch 5 bis 10 Minuten zu investieren. Damit lag die mittlere Beratungsdauer tendenziell sogar etwas niedriger als in Phase I.

Bei der Selbsteinschätzung von Kompetenz und eigenen Fähigkeiten hinsichtlich der Tabakentwöhnungsberatung wurden bei den entsprechenden Fragen sechsstufige Skalen verwendet. Die in Tabelle 7 angegebenen Prozentzahlen beziehen sich auf den Anteil der Befragten, der bei den entsprechenden Fragebogen-Items eine der beiden positiven Skalenoptionen auswählte. 
Eine hohe Kompetenz bei der Raucherberatung äußerten 3 Mitarbeiter in Phase I und 8 in Phase II $(\mathrm{p}=0,082)$. Gute Kenntnisse bezüglich der Nikotinabhängigkeit gaben 8 Pfleger in Phase I und 5 in Phase II ( $\mathrm{p}=0,322)$ an, gute Kenntnisse der gesundheitlichen Folgen des Rauchens wurden in beiden Phasen von 17 Pflegern genannt und gute Kenntnisse der pharmakologischen sowie verhaltenstherapeutischen Therapiemöglichkeiten äußerten 4 Mitarbeiter in Phase I und 9 in Phase II $(p=0,099)$.

Die eigene Fähigkeit, Raucher zu einem Rauchverzicht zu motivieren, schätzten in Phase I drei Mitarbeiter und in Phase II fünf Mitarbeiter als hoch ein $(\mathrm{p}=0,434)$.

Tab. 7: Ergebnisse der Mitarbeiterbefragung, angegeben als prozentualer Anteil (absolute Zahlen)

\begin{tabular}{|c|c|c|c|}
\hline & $\begin{array}{l}\text { Phase I } \\
(\mathrm{n}=22)\end{array}$ & $\begin{array}{l}\text { Phase II } \\
(\mathrm{n}=22)\end{array}$ & $p-$ Wert \\
\hline Teilnahme an der Mitarbeiterschulung & - & $54,5(12)$ & - \\
\hline \multicolumn{4}{|l|}{ Rauchstatus } \\
\hline Nichtraucher & $45,5(10)$ & $45,5(10)$ & \multirow{3}{*}{1} \\
\hline Ex-Raucher & $31,8(7)$ & $31,8(7)$ & \\
\hline Raucher & $22,7(5)$ & $22,7(5)$ & \\
\hline \multicolumn{4}{|c|}{ Anteil beratener Raucher/innen } \\
\hline$<5 \%$ & $81,8(18)$ & $59,1(13)$ & \\
\hline $5-25 \%$ & $13,6(3)$ & $36,4(8)$ & \\
\hline $25-50 \%$ & $4,5(1)$ & $0(0)$ & \\
\hline $50-75 \%$ & $0(0)$ & $0(0)$ & \\
\hline$>75 \%$ & $0(0)$ & $4,5(1)$ & \\
\hline \multicolumn{4}{|c|}{$\begin{array}{l}\text { Selbsteinschätzung von Wissen und Fähigkeiten bezüglich der Beratung } \\
\text { von Rauchern/innen }\end{array}$} \\
\hline $\begin{array}{l}\text { Hohe Kompetenz bei der Beratung von Rauchern/ } \\
\text { Raucherinnen }\end{array}$ & $13,6(3)$ & $36,4(8)$ & 0,082 \\
\hline $\begin{array}{l}\text { Umfassendes Wissen über die Grundlagen der Ta- } \\
\text { bakabhängigkeit }\end{array}$ & $36,4(8)$ & $22,7(5)$ & 0,322 \\
\hline $\begin{array}{l}\text { Umfassendes Wissen über die Folgeerkrankungen } \\
\text { des Rauchens }\end{array}$ & $77,3(17)$ & $77,3(17)$ & 1 \\
\hline $\begin{array}{l}\text { Umfassende Kenntnisse der verhaltensbezogenen } \\
\text { und medikamentösen Maßnahmen zur Unter- } \\
\text { stützung der Tabakentwöhnung }\end{array}$ & $18,2(4)$ & $40,9(9)$ & 0,099 \\
\hline $\begin{array}{l}\text { Ausgeprägte Fähigkeit, die Raucher/innen zum } \\
\text { Rauchverzicht zu motivieren }\end{array}$ & $13,6(3)$ & $22,7(5)$ & 0,434 \\
\hline
\end{tabular}




\begin{tabular}{|c|c|c|c|}
\hline \multicolumn{4}{|c|}{ Durchschnittliche Dauer einer Entwöhnungsberatung } \\
\hline $0-5 \mathrm{~min}$ & $36,4(8)$ & $50,0(11)$ & \\
\hline $5-10 \mathrm{~min}$ & $22,7(5)$ & $36,4(8)$ & \\
\hline $10-20 \mathrm{~min}$ & $13,6(3)$ & $9,1(2)$ & \\
\hline$>20 \mathrm{~min}$ & $9,1(2)$ & $0(0)$ & \\
\hline Fehlende Angaben & $18,2(4)$ & $4,5(1)$ & \\
\hline \multicolumn{4}{|c|}{ Reaktionen der Raucher/innen auf die Beratung } \\
\hline Patient zeigte sich interessiert & & $18,2(4)$ & \\
\hline Patient wünschte zusätzliche Informationen & & $9,1(2)$ & \\
\hline Patient war zum Rauchstopp bereit & & $4,5(1)$ & \\
\hline Patient gab das Rauchen auf & & $4,5(1)$ & \\
\hline Patient lehnte die Beratung ab & & $40,9(9)$ & \\
\hline \multicolumn{4}{|c|}{ Wahrnehmung der SOP } \\
\hline $\begin{array}{l}\text { Beratung konnte gut in den Arbeitsalltag integriert } \\
\text { werden }\end{array}$ & & $27,3(6)$ & \\
\hline Beratung wurde Teil der täglichen Routine & & $18,2(4)$ & \\
\hline $\begin{array}{l}\text { Die SOP sollte auch auf anderen Stationen einge- } \\
\text { setzt werden }\end{array}$ & & $54,5(12)$ & \\
\hline $\begin{array}{l}\text { Ich kann mir vorstellen, auch andere Mitarbeiter } \\
\text { zur Beratung rauchender Patienten zu motivieren }\end{array}$ & & $22,7(5)$ & \\
\hline
\end{tabular}

In Bezug auf die zusätzliche Arbeitsbelastung und die praktische Durchführung der SOP gaben 6 Mitarbeiter an, die Beratung rauchender Patienten gut in den Arbeitsalltag integrieren zu können. Bei 4 Pflegern wurde die Intervention bei den Rauchern ein fester Bestandteil der täglichen Routine.

Zwölf Mitarbeiter waren der Meinung, man solle die SOP auch auf anderen Stationen einführen. Fünf Mitarbeiter konnten sich vorstellen, auch andere Kollegen zur Entwöhnungsberatung rauchender Patienten zu motivieren.

Die Pflegekräfte wurden außerdem gebeten, anzugeben, welche Reaktionen ihnen von den Patienten während des Beratungsgespräches entgegengebracht wurden. Gewertet wurden jeweils die beiden positiven bzw. die beiden negativen Optionen der sechsstufigen Skalen.

Vier Pflegekräfte vernahmen ein hohes Interesse an der Entwöhnungsberatung seitens der Raucher, von denen einige über die Beratung hinaus noch zusätzliche Informationen zur Tabakentwöhnung wünschten. Fast die Hälfte der befragten Pflegekräfte gab an, dass die Raucher ein entsprechendes Beratungsgespräch ablehnten. 


\subsection{Auswertung der Mitarbeiter-Interviews}

Zur Teilnahme an den im Anschluss an Phase II durchgeführten Einzelinterviews erklärten sich insgesamt 10 Pflegerinnen und Pfleger sowie 5 ärztliche Mitarbeiter, die während Phase II auf den betreffenden Studienstationen eingesetzten waren, bereit.

Die Darstellung dieses Kapitels erfolgt in Anlehnung an das „COM- $B^{“}$ - Modell (siehe Kapitel 1.7) und unterteilt sich demgemäß in die drei dort definierten Hauptdeterminanten des Verhaltens: Die Fertigkeiten (capability), die Gelegenheit (opportunity) und die Motivation (motivation). Alle im Zuge der Auswertung der transkribierten Interviews formulierten Themen (themes) sind, ebenfalls unter Zuordnung zu einem dieser drei Aspekte des Verhaltens, in Tab. 8 übersichtsweise aufgeführt.

\section{Motivation}

Die deutliche Mehrheit der Pflegekräfte ebenso wie alle interviewten Ärzte empfanden die eingeführte SOP als wichtig und notwendig. Einige betonten, nunmehr einen strukturierteren Zugang in Bezug auf die Tabakentwöhnung der hospitalisierten Raucher zu haben. So schätzte ein ärztlicher Mitarbeiter die SOP ,[...] als eine sehr sinnvolle und wertvolle Maßnahme ein, da [...] die Raucherentwöhnung bei kardiovaskulären oder auch pneumologischen Erkrankungen doch manchmal etwas zu kurz gekommen ist als therapeutische Option und SOPs ja immer dazu geeignet sind, Handeln zu strukturieren und einem auch einen Leitfaden an die Hand zu geben“. Eine Pflegekraft schätze die Notwendigkeit der SOP gering ein, da die Liegezeit der Patienten nicht besonders lang sei.

Trotz der Zustimmung zur SOP ließen einige Mitarbeiter verlauten, dass die Handlungsanweisungen nur unvollständig umgesetzt wurden. So erhielt nicht jeder identifizierte Raucher einen Ratschlag zum Rauchverzicht, wie im Rahmen der SOP gefordert. Stattdessen gaben manche Mitarbeiter eine eher selektive Umsetzung diesbezüglich an, wonach das Thema Rauchen durch sie nur sporadisch angesprochen wurde mit Fokussierung auf die Beratung jüngerer Patienten, bei denen angeblich eine höhere Chance der positiven Beeinflussung der Gesundheit bestünde. Andere Pflegekräfte erteilten bei Gelegenheitsrauchern keinen Ratschlag zum Rauchstopp oder unterließen sämtliche Beratungsaktivitäten, sobald ein Patient während des initialen Kontaktes einen fehlenden Willen zeigte. Eine weitere Pflegekraft wurde nur aktiv, wenn ein Raucher gezielt danach fragte. 
Auch unter den Ärzten konnte eine unvollständige Umsetzung beobachtet werden. So setzte ein ärztlicher Mitarbeiter sein Beratungsgespräch nur dann fort, wenn der Patient ein gewisses Interesse zeigte.

Acht Pflegekräfte und zwei ärztliche Mitarbeiter konstatierten, nach Einführung der SOP keine besonderen Veränderungen im Stationsalltag wahrgenommen zu haben. Einige kritisierten ein fehlendes Feedback in Bezug auf die Effekte der implementierten Maßnahmen. In diesem Zusammenhang äußerte ein Arzt, er könne sich auch vorstellen, dass eine Rückmeldung über die Erfolge der SOP „[...] die Motivation der Kollegen nochmal steigern würde, wenn man sieht, dass da tatsächlich was bei rumkommt.“

Als positiven Aspekt bemerkten drei ärztliche Mitarbeiter, dass durch die Implementierung der SOP die Wahrnehmung des Rauchens als ein wichtiges Anliegen insgesamt gesteigert wurde.

Einen maßgeblichen Einfluss auf die Motivation als verhaltensdeterminierenden Aspekt und folglich mit direktem Einfluss auf das Verhalten ist die sogenannte Rollen-Kongruenz. Sie meint in diesem Kontext, inwieweit das medizinische Personal die Tabakentwöhnung rauchender Patienten als berufliche Aufgabe und Verpflichtung ansieht.

Während sich ungefähr die Hälfte aller Befragten darüber einig war, dass alle medizinischen Mitarbeiter eine Rolle bei der Entwöhnung von hospitalisierten Rauchern spielen würden, vermerkten 5 Pflegekräfte und 4 Ärzte, dass die konkrete Ansprache des Themas Rauchen in den primären Aufgabenbereich eines Arztes bzw. einer Ärztin fallen würde. Vor allem einige pflegerische Mitarbeiter begründeten dies mit dem höheren Stellenwert des Arztes aus Patientensicht und meinten, ein ärztlicher Ratschlag würde ernster genommen werden. Eine andere Pflegekraft hingegen hob hervor, dass eine Beratung seitens der Pflege auch Vorteile hätte: Die Pflegekräfte würden nämlich deutlich mehr Zeit mit dem Patienten verbringen und hätten auf diese Weise einen leichteren Zugang, das Rauchen zu thematisieren.

Die Mehrheit der Ärzte konnte sich mit ihren Aufgaben, beispielsweise der Aufklärung über tabakrauchassoziierte Gesundheitsrisiken oder dem Anbieten von Unterstützung bei einem Abstinenzversuch, identifizieren. Unter den Pflegekräften bekannten sich die meisten dazu, dass die Dokumentation des Rauchstatus ebenso wie ein kurzer Ratschlag zum Rauchverzicht in ihren Aufgabenbereich fallen würde.

Einige wenige Pfleger hatten das Gefühl, die jeweiligen Aufgaben wären nicht eindeutig verteilt und geregelt und würden zum Teil auch einfach auf andere Mitarbeiter abgeschoben. Außerdem dem Aspekt Rollenkongruenz zuzuordnen ist die Aussage eines Arztes, die Betreuung 
rauchender Patienten könne in der Klinik insgesamt nur „vage in die Wege geleitet werden“, dies sei nämlich die vordergründige Aufgabe des Hausarztes. Als weiteren Kritikpunkt bescheinigte er einigen Kollegen eine geringe intrinsische Motivation bei der Tabakentwöhnung hospitalisierter Raucher.

Die oben dargestellten Punkte fallen unter die reflektierte Motivation, also bewusste kognitive Prozesse wie Pläne und Bewertungen. Davon abzugrenzen ist die automatische Motivation, die überwiegend unbewusste Aspekte wie Impulse und Emotionen umfasst.

Hierunter einzuordnen sind unter anderem die Erfahrungen und Gefühle, die bei dem Personal im Rahmen der Entwöhnungsgespräche aufkamen. So empfand eine Pflegekraft die Gespräche als frustrierend und bemerkte, selten auf eine positive Resonanz seitens der beratenen $\mathrm{Pa}$ tienten zu stoßen. Einer anderen schien es unangenehm, das Rauchen anzusprechen, da sich der Raucher „abgestempelt“ oder gar „auf den Schlips getreten“ fühlen könnte. Ein weiterer hatte das Gefühl, dem Patienten das Thema aufzuzwängen. Zwei Mitarbeiter, ein Arzt und ein Pfleger, äußerten insgesamt ein positives Gefühl während der Gespräche. Ein anderer Arzt zeigte sich enttäuscht und leicht verärgert, wenn ein Patient die Vorschläge zum Rauchverzicht ablehnte, weiter rauchte und so „seine Gesundheit verspielte“.

Die berichteten Reaktionen der Patienten während der Entwöhnungsinterventionen fielen sehr gemischt aus. Die Pflegekräfte beobachteten vereinzelt eine ,,ablehnende Haltung“ bei der Thematisierung des Rauchens durch die Patienten, andere machten positive Erfahrungen und hatten das Gefühl, manche Raucher seien ,ganz glücklich darüber“،. Unter den ärztlichen Mitarbeiten herrschte der überwiegende Eindruck, dass sich die meisten Raucher einsichtig zeigten und die Notwendigkeit einer Tabakentwöhnung für ihre weitere Gesundheit erkannten, insbesondere wenn sie von einem akuten und schweren Ereignis wie einem Myokardinfarkt betroffen waren.

Dennoch gab es Patienten, die ihren Rauchstopp alleine bewerkstelligen wollten und auf eine begleitende Medikation oder die Anbindung an die hausinterne Entwöhnungsambulanz verzichteten. Auch hier beobachteten zwei Ärzte in einigen Fällen einen Zusammenhang mit der Schwere des Ereignisses, welches zur Klinikaufnahme des Rauchers führte: „[...] unter dem Eindruck von einem frischen Infarkt sind die meisten überzeugt, dass sie es irgendwie selber schaffen“. Die akute Erkrankung wurde von dem Patienten demzufolge als so „einschneidend“" empfunden, dass jegliche Hilfe für nicht notwendig gehalten wurde. 
In Bezug auf die Hindernisse auf dem Weg zu einer adäquaten Raucherversorgung wurde nicht nur die Überschätzung der eigenen Willenskraft durch die Raucher erwähnt. Als problematisch wurde zusätzlich das Rauchverhalten der Mitarbeiter angesehen, insbesondere wenn dies ,direkt auf dem Stationsbalkon“ vor den Augen der Patienten geschehe, die zuvor möglicherweise hinsichtlich einer Entwöhnung beraten wurden.

Fünf der zehn interviewten Pflegekräfte war der Meinung, Rauchen sei eine persönliche Angelegenheit und „Privatsache“ (,[...] im Prinzip sind die alle alt genug, um das selber zu wissen. Dass Rauchen schlecht ist, das ist ja jetzt nicht unbekannt".) Vor diesem Hintergrund zweifelte eine Pflegekraft sogar den Stellenwert der Raucherversorgung durch die Universitätsklinik Göttingen an: „Ich finde das nicht so wichtig, denn hier werden wichtigere Sachen behandelt als das Rauchen. Es ist ja ein persönliches Suchtverhalten“.

Alle ärztlichen Mitarbeiter einschließlich einiger Pflegekräfte bemerkten ferner, nicht den kompletten Inhalt der implementierten SOP zu kennen. Außerdem wurde eine im Laufe der Zeit generell abnehmende Tendenz der Aktivitäten bezüglich der Raucherentwöhnung festgestellt. So vernahmen mehrere Mitarbeiter, dass unmittelbar nach der Schulungsmaßnahme alles ,gut funktioniert“ habe, während die Entwöhnungsaktivitäten ebenso wie die Kenntnisse über die Inhalte und Abläufe der SOP mit der Zeit abnahmen und bei manchen immer mehr ,in den Hintergrund rückten“.

\section{Fertigkeiten}

Dieser Teil stellt die Bemerkungen und Äußerungen der Mitarbeiter dar, die das Wissen und die praktischen Fähigkeiten in Bezug auf die Entwöhnungsbehandlung hospitalisierter Raucher sowie die zur Vermittlung dieser Fähigkeiten durchgeführte Mitarbeiterschulung betreffen.

In Anlehnung an den letztgenannten Punkt des vorangegangenen Kapitels, also die verbreitete Wahrnehmung abnehmender Entwöhnungsaktivitäten unter den Mitarbeitern, kann die positive Einstellung der Mehrheit der Interviewten gegenüber einer erneuten Trainingsmaßnahme betrachtet werden. So äußerten 9 Pflegekräfte und 3 Ärzte, darunter einige mit direktem Verweis auf bestehende Unsicherheiten bezogen auf die Anwendung der Medikamente oder die Inhalte der SOP im Allgemeinen, dass sie eine weitere Schulung für hilfreich erachten würden. Dazu ein pflegerischer Mitarbeiter: „Die Schulung, an der ich teilgenommen habe, fand ich sehr gut. Wenngleich ich auch behaupten würde, dass es notwendig wäre, dass so etwas ab 
und an wieder aufgefrischt wird. Weil [...] da eben doch so viele Unsicherheiten herrschen $[\ldots]^{\prime \prime}$.

Acht Pflegekräfte forderten zusätzliche Trainingsmaßnahmen speziell zum Medikamenteneinsatz, vier weitere wollten Beratungs- und Gesprächstechniken vertiefen. Auch unter den Ärzten gab es einen erkennbaren Bedarf vor allem an Schulungen bezüglich der Pharmakotherapie. Die angesprochenen Unsicherheiten bezogen sich vordergründig auf die Dosierungen der verschiedenen Nikotinersatzmittel sowie auf potenzielle Nebenwirkungen und Kontraindikationen besonders bei KHK-Patienten. Entsprechend schätzten vier der fünf interviewten Ärzte ihre Kompetenzen bezüglich der Pharmakotherapie als niedrig ein. Zwei gaben an, überhaupt keine Erfahrung in diesem Punkt zu haben.

Unter den Pflegekräften war weniger als die Hälfte der Ansicht, die eingesetzten Medikamente wären effektiv im Rahmen der Tabakentwöhnung. Drei Pfleger äußerten explizit, die Präparate würden nicht helfen oder seien unnötig: ,[...] die Medikamente halte ich persönlich für nicht sinnvoll. Weil es eine reine Disziplinsache ist und vom Kopf kommen muss“". Acht der zehn befragten Pflegekräfte beurteilten sich wenig bis gar nicht kompetent in Bezug auf ihre Anwendung.

Bei der Einschätzung der Fertigkeiten im Hinblick auf die Entwöhnungsberatungen fühlten sich vier Pflegekräfte und drei ärztliche Mitarbeiter kompetent, das Thema Rauchen mit dem Patienten zu diskutieren und eine Entwöhnung zu initiieren. Zwei Ärzte gaben an, ihre Kompetenzen in diesem Bereich seien weniger gut ausgeprägt, vor allem im Vergleich mit ihren Fähigkeiten auf dem Gebiet der Behandlung der anderen kardiovaskulären Risikofaktoren: „Es ist unverhältnismäßig, dass man so viel weiß über die Einstellung und medikamentöse Behandlung [...] anderer Risikofaktoren, aber demgegenüber ist es unverhältnismäßig wenig Wissen eigentlich, was die Raucherentwöhnung und da auch die medikamentöse Therapie speziell betrifft".

\section{Gelegenheit}

Unter den Pflegekräften wurde anerkannt, dass sich ihnen durch den erhöhten Patientenkontakt wiederholt Gelegenheit bieten würde, das Rauchen mit dem Patienten thematisieren zu können. Eine vollständige Umsetzung der SOP einschließlich der Erläuterung der Handhabung der Nikotinersatzmedikamente konnte laut einiger Pfleger hingegen nur unzureichend realisiert werden, da die entsprechenden Präparate von manchen Ärzten aufgrund von Unsi- 
cherheiten bezüglich der Dosierung oder auch generell nicht bzw. nur zögerlich verordnet wurden. Einfluss auf die begrenzte Anwendung der Nikotinersatzmittel hätte aber auch die mangelnde Bereitstellung der Medikamente auf den jeweiligen Stationen, vor allem zu Studienbeginn. Dies wurde gleichermaßen von den ärztlichen Mitarbeitern kritisiert.

Von einer Pflegekraft wurde ferner angeregt, die SOP sowie die Informationen zur Nikotinersatztherapie auch im Intranet zu Nachschlagezwecken aufzuführen.

Auch bei den ärztlichen Mitarbeitern würden sich im Arbeitsalltag Gelegenheiten bieten, Beratungen zum Rauchstopp durchzuführen oder zu initiieren, sei es bei der täglichen Visite oder bei spezifischen Untersuchungen. Zwei Ärzte wiesen auf die zum Teil kurzen Liegezeiten der Patienten hin, insbesondere auf der Intermediate Care - Station 1025, was eine adäquate Raucherentwöhnung erschweren würde: ,[...] auf der Station sind die Patienten ja nur kurz. Dann spricht man es einmal an und dann verliert man sie aus den Augen. Also die Kontinuität ist vielleicht noch optimierbar bis zur Entlassung“.

Zudem von beiden Seiten der Mitarbeiter angemerkt wurde die Tatsache, dass auf den Studienstationen neu eingesetztes Personal, also bei einem Arbeitseinsatz in der Zeit nach der Implementierungs- bzw. Schulungsphase, nicht mit der SOP und den entsprechenden Maßnahmen vertraut sei.

Sechs der zehn interviewten Pflegekräfte maßen der Raucherentwöhnung einen hohen Stellenwert im Rahmen der kardiologischen Behandlung bei. Gleichzeitig äußerte die Hälfte von ihnen, innerhalb der Universitätsklinik Göttingen (UMG) spiele diese insgesamt betrachtet eine eher untergeordnete Rolle: „Im Klinikum selbst hat sie im Moment glaube ich noch einen geringen Stellenwert. [...] Was die Kardiologie betrifft, finde ich, hat es mittlerweile einen höheren Stellenwert erlangt, wenngleich wir da auch sicherlich noch nicht ganz oben angekommen sind. Ich denke mir, da ist noch Spielraum.“ Einige Mitarbeiter erkannten dennoch eine Tendenz, in der der Tabakentwöhnung eine immer größere Bedeutung zukomme.

Kritik wurde dahingehend geäußert, dass nach wie vor sowohl für Patienten als auch für das medizinische Personal die Möglichkeit bestünde, an der UMG zu rauchen: „Wir akzeptieren das Rauchen zum einen und wir ermöglichen es zum anderen ja auch. Also Patienten, die gehfähig sind, [...] dürfen ja sogar auf dem Balkon rauchen.“

Mehrere pflegerische Mitarbeiter gewannen den Eindruck, für viele Patienten sei das Thema Rauchen lediglich von geringerer Priorität. So würden sich viele Raucher nicht „,besonders dafür interessieren“, hätten eher „,mit anderen Sachen zu tun“ und „fragten auch nicht nach“ ei- 
ner Entwöhnung oder Unterstützung. Außerdem wüssten „die meisten ja auch, dass Rauchen ungesund ist“ und seien „bestimmt auch schon zum x-ten Mal angesprochen worden.“

Für vier der zehn Pflegekräfte war der mangelnde Zeitfaktor im Rahmen der ohnehin sehr umfangreichen Stationsarbeit ausschlaggebend für eine unzureichende Betreuung rauchender Patienten bzw. verantwortlich für eine unvollständige Durchführung der zusätzlich anfallenden SOP-spezifischen Aufgaben im Allgemeinen: „[...] viele fühlen sich jetzt schon sehr stark gefordert beim Arbeiten und wenn dann noch was dazukommt, das machen viele dann einfach nicht.““

Ärztlicherseits wurde in einem Fall ebenfalls ein erhöhter Zeitaufwand durch die implementierte SOP berichtet, ein anderer konnte dies nicht feststellen. Dieser bemerkte dennoch, dass die Handlungsrichtlinien auf seiner Station nicht ausgehängt wurden bzw. nicht leicht zugänglich waren.

Tab. 8: Darstellung der im Rahmen der Auswertung der transkribierten Interviews ermittelten Themen. Die ärztlichen Mitarbeiter $(n=5)$ boten 149 Zitate, unterteilt in 16 Themen, die pflegerischen Mitarbeiter ( $n=10)$ insgesamt 269 Zitate, gruppiert in 18 Themen.

\begin{tabular}{|c|c|c|c|}
\hline \multirow{2}{*}{$\begin{array}{c}\text { Aspekt des } \\
\text { COM-B } \\
\text { Modells }\end{array}$} & \multirow[b]{2}{*}{ Thema } & \multicolumn{2}{|c|}{ Anteil der Zitate } \\
\hline & & $\begin{array}{l}\text { Ärzte } \\
(149 \mathrm{Zi}- \\
\text { tate })\end{array}$ & $\begin{array}{l}\text { Pfleger } \\
(269 \mathrm{Zi}- \\
\text { tate })\end{array}$ \\
\hline \multirow{5}{*}{ 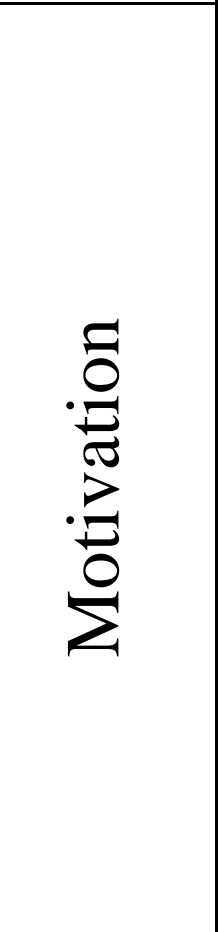 } & $\begin{array}{l}\text { Bewertung der SOP (z.B. "Die SOP ermöglicht einen } \\
\text { strukturierteren Ansatz zur Dokumentation des } \\
\text { Rauchstatus" }\end{array}$ & $17,4 \%$ & $10,4 \%$ \\
\hline & $\begin{array}{l}\text { Partielle Umsetzung der SOP (z.B. Beratung ledig- } \\
\text { lich der Gelegenheitsraucher oder interessierter Rau- } \\
\text { cher }\end{array}$ & $4,0 \%$ & $5,9 \%$ \\
\hline & $\begin{array}{l}\text { Auswirkungen der SOP auf den Alltag (z.B. Feststel- } \\
\text { len keinerlei Änderungen nach Implementierung der } \\
\text { SOP) }\end{array}$ & $6,0 \%$ & $4,1 \%$ \\
\hline & $\begin{array}{l}\text { Rollenkongruenz (z.B. "Es ist die primäre Aufgabe } \\
\text { des Arztes, das Rauchen bei dem Patienten anzu- } \\
\text { sprechen") }\end{array}$ & $10,7 \%$ & $18,2 \%$ \\
\hline & $\begin{array}{l}\text { Generelle Einstellung zum Rauchen sowie zu den } \\
\text { Entwöhnungsmaßnahmen (z.B. "Rauchen ist eine } \\
\text { persönliche Angelegenheit") }\end{array}$ & $1,3 \%$ & $2,2 \%$ \\
\hline
\end{tabular}




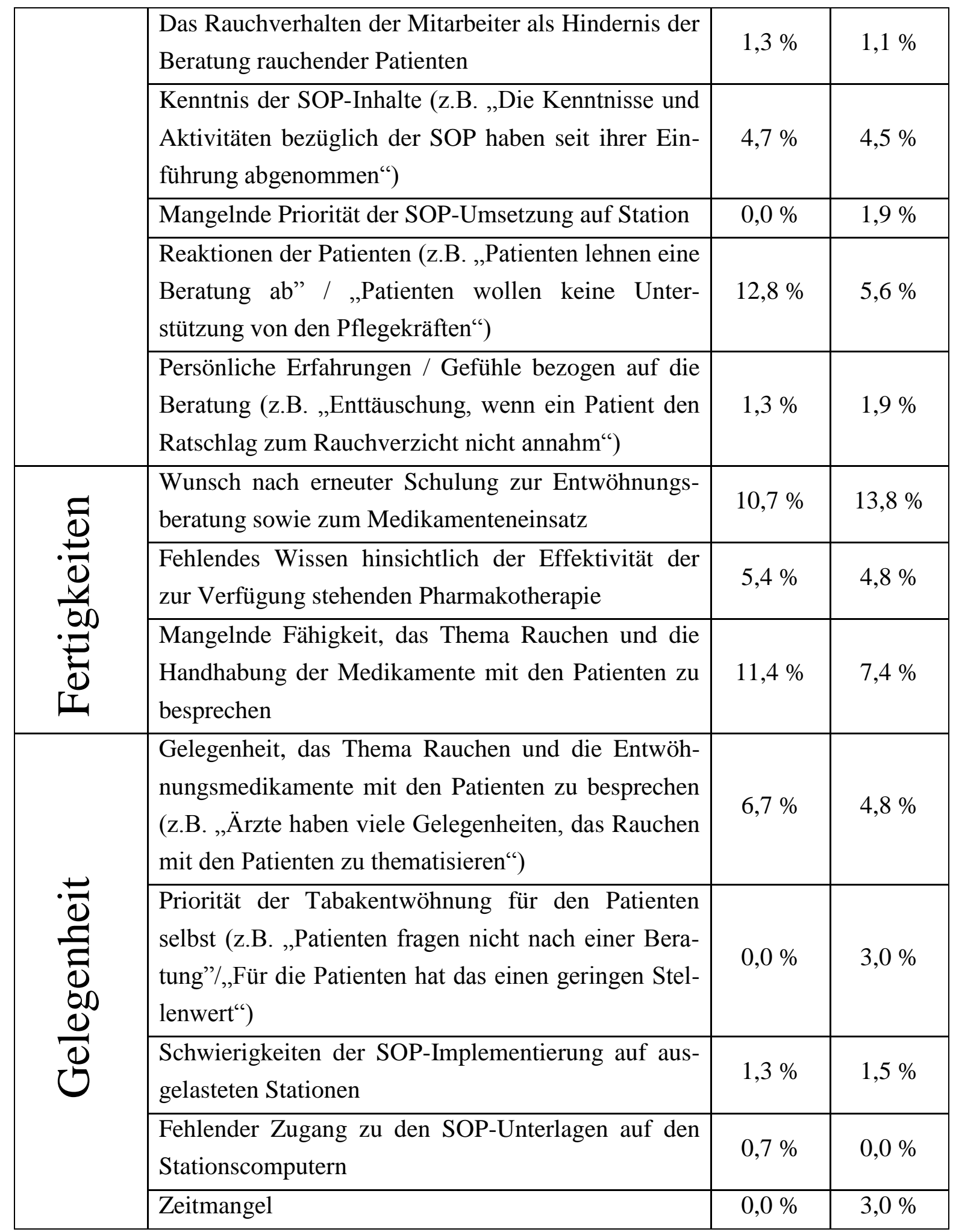




\section{Diskussion}

\subsection{Zusammenfassung der wesentlichen Ergebnisse}

Im Rahmen der Einführung von Standard Operating Procedures zum leitlinienkonformen Management rauchender Patienten sowie der parallel dazu stattfindenden Mitarbeiterschulung konnte gemäß der Angaben aus der Patientenbefragung eine signifikante Erhöhung der Interventionshäufigkeit bei hospitalisierten Rauchern seitens des ärztlichen Personals, nicht jedoch durch die pflegerisch tätigen Mitarbeiter erzielt werden. Insgesamt betrachtet lag der Anteil beratener Patienten auch nach der SOP-Implementierung unter $40 \%$. Die durchgeführten Beratungsgespräche zum Rauchverzicht wurden von der Mehrheit der Patienten positiv aufgenommen.

Obwohl in Phase I nahezu alle und in Phase II alle 75 teilnehmenden Raucher eine Reduktion des Tabakkonsums oder gar einen vollständigen Rauchverzicht unternahmen, lag die Inanspruchnahme von Hilfen diesbezüglich weiterhin auf einem niedrigen Niveau. So griff kein Raucher aus der ersten Studienphase und nur fünf Raucher (6,7 \%) aus Phase II auf die unterstützende Nikotinersatztherapie zurück. Auch die Anbindung an die klinikeigene Entwöhnungsambulanz konnte nach Implementierung der SOP nicht wesentlich gesteigert werden.

Aus den Ergebnissen der schriftlichen Mitarbeiterbefragung war ein, wenn auch geringer, Trend zu mehr Beratungen erkennbar, der allerdings nicht mit einer längeren Beratungsdauer einherging.

Der qualitative Studienteil (Einzelinterviews mit ärztlichen und pflegerischen Mitarbeitern) ermöglichte die Identifikation von Hindernissen auf dem Weg zu einer adäquaten Raucherversorgung.

Dabei ließ sich der überwiegende Teil dieser Barrieren dem Aspekt der Motivation zuschreiben. So zeigte sich, dass trotz der breiten Zustimmung zur SOP einschließlich ihrer empfundenen Notwendigkeit unter vielen Mitarbeitern eine lediglich unvollständige Umsetzung der SOP-spezifischen Maßnahmen praktiziert wurde, wonach beispielsweise nur interessierte Raucher beraten wurden oder Gelegenheitsraucher gänzlich von einer Intervention ausgenommen wurden. Ebenfalls konnte eine mangelhafte Rollenkongruenz aufgezeigt werden. Die Mehrheit der interviewten Mitarbeiter war der Ansicht, die Thematisierung des Rauchens falle in den primären Aufgabenbereich des ärztlichen Personals. Die Hälfte der Pflegekräfte ver- 
trat zudem die Meinung, Rauchen sei Privatsache bzw. eine persönliche Angelegenheit der Patienten.

Andere im Rahmen der Interviews ermittelte Hindernisse fielen unter den Aspekt der Fertigkeiten. So gaben $40 \%$ der Pflegekräfte und $60 \%$ Ärzte eine hohe Kompetenz bezüglich der Raucherberatung an. Deutlich weniger versiert schätzten sich die meisten Mitarbeiter in Bezug auf den Umgang mit den Entwöhnungsmedikamenten ein. Hier zeigten sich große Unsicherheiten was Einsatz, Dosierung, Nebenwirkungen und Handhabung der Präparate betrifft. Mehr als die Hälfte der Pflegekräfte hielt die eingesetzten Präparate zur medikamentösen Behandlung der Tabakabhängigkeit für nicht effektiv.

Nicht nur aufgrund der verbreiteten Defizite hinsichtlich des Medikamenteneinsatzes sondern ebenfalls angesichts allgemein abnehmender Entwöhnungsaktivitäten mit zunehmendem zeitlichen Abstand zur Trainingsmaßnahme befürwortete die große Mehrheit der Befragten eine erneute Schulung.

Dem Begriff der Gelegenheit zuzuordnen war die von vielen Mitarbeitern geäußerte Anerkennung des zunehmenden Stellenwerts der Behandlung der Tabakabhängigkeit innerhalb des kardiologischen Settings ebenso wie die Tatsache, dass sich während des stationären Aufenthaltes wiederholt Gelegenheit bieten würde, das Thema Rauchen anzusprechen. Einige wiesen auf die mangelnde Zeit hin, andere stellten als Hindernis eine unzureichende Priorisierung des Rauchens durch den Patienten heraus oder berichteten von einer ablehnenden Haltung der Raucher im Rahmen einer Entwöhnungsintervention, was manche Mitarbeiter von zukünftigen Entwöhnungsaktivitäten entmutigte.

Kritik wurde ferner dahingehend geäußert, dass für das medizinische Personal trotz der rauchfreien Umgebung des Klinikums weiterhin die Möglichkeit bestünde, auf dem Stationsbalkon $\mathrm{zu}$ rauchen.

\subsection{Limitationen der Studie}

Die vorliegende Studie weist einige Limitationen auf, die im Folgenden dargestellt werden:

Zunächst muss konstatiert werden, dass keine biochemische Validierung des Rauchstatus der Patienten erfolgte. Zum einen ließ das große Einzugsgebiet der Universitätsklinik Göttingen eine geringe Teilnahme an den dafür notwenigen Cotinin- oder CO-Tests erwarten, die eine Wiedervorstellung der Studienteilnehmer an der UMG nach Ablauf von mindestens sechs 
Monaten erfordert hätte. Zum anderen wurde nicht die Tabakabstinenz als Studienendpunkt gewählt, sondern der durch die implementierten Maßnahmen (SOP und Schulung) induzierte Effekt auf die Interventionshäufigkeit seitens des medizinischen Personals.

Bei der Betrachtung gruppenspezifischer Unterschiede in Bezug auf den Abhängigkeitsgrad der Raucherinnen und Raucher, welcher mit Hilfe des Fagerstrom-Tests für Nikotinabhängigkeit (FTND) ermittelt wurde, zeigte sich unter den Studienteilnehmern der ersten Projektphase ein insgesamt höherer Abhängigkeitswert $(5,0 \pm 2,2)$ verglichen mit den Teilnehmern aus Phase II $(3,7 \pm 2,1)$. So ließe sich einerseits annehmen, die Patienten aus Phase II seien aufgrund ihrer weniger starken Abhängigkeit möglicherweise zugänglicher und empfänglicher für Ratschläge zum Rauchverzicht oder andere Tabakentwöhnungsinterventionen. Zumindest liefert die Studienlage dahingehend Hinweise, dass ein niedriger Abhängigkeitswert nach dem FTND mit einer höheren Abstinenz korreliert (Bhang et al. 2013, Fagerstrom et al. 2012, Ferguson et al. 2003).

Auf der anderen Seite wurden deutlich mehr Studienteilnehmer aus Phase I (45,3 \% vs. 30,7 $\%)$ mit einem akuten Koronarsyndrom aufgenommen. Auch hier scheint es einen Zusammenhang mit der Empfänglichkeit der Raucher bezüglich Entwöhnungsmaßnahmen zu geben, wonach bei aufgrund eines kardiovaskulären Geschehens hospitalisierten Rauchern ein höherer Effekt einer intensiven Beratung beobachtet wurde (Rigotti et al. 2008). Somit könnte man eine gesteigerte Zugänglichkeit für die Entwöhnungsinterventionen unter den Probanden aus Phase I annehmen, so dass dieser Bias den oben genannten konterkariert. In welchem Ausmaß sich dies im Einzelnen manifestiert und ob sich beide Effekte letztendlich ausgleichen, kann abschließend nicht exakt beurteilt werden.

Eine vollständige und lückenlose Befragung aller auf den kardiologischen Studienstationen beschäftigten ärztlichen und pflegerischen Mitarbeiter war im Rahmen der hier vorgestellten Studie leider nicht möglich. Hieraus können Einschränkungen in Bezug auf die Repräsentativität resultieren, wonach die aus dieser Stichprobe generierten Erkenntnisse bezüglich der praktizierten Handlungen, der generellen Einstellungen und Denkweisen letztendlich nicht für alle Mitarbeiter der UMG zutreffen. Ebenfalls mag eine unmittelbare Übertragung und Anwendung der ermittelten Studienergebnisse auf andere Kliniken und Institutionen sowohl innerhalb als auch außerhalb der Bundesrepublik Deutschland nicht uneingeschränkt möglich sein. 
In diesem Zusammenhang muss berücksichtigt werden, dass die Teilnahme an den Mitarbeiter-Interviews auf freiwilliger Basis erfolgte. Ein möglicher Selektionsbias, wonach insbesondere die auf dem Gebiet der Tabakentwöhnung engagierten und interessierten Mitarbeiter an der Befragung teilnahmen, ist nicht auszuschließen.

Eine weitere Limitation betrifft den Effekt der implementierten Maßnahmen, insbesondere den der Schulungsmaßnahme. Durch die nicht vollzählige Beteiligung der Pflegekräfte sowie der Abwesenheit der ärztlichen Mitarbeiter mag der durch die anschließenden Erhebungen in Phase II ermittelte Effekt der Schulung entsprechend geringer ausgefallen sein. In diesem Zusammenhang wäre es durchaus von Interesse, die Gründe des Personals für ihr Fernbleiben von der Fortbildungsveranstaltung zu erfahren. Bezüglich des „Fortbildungsverhaltens“ könnte wiederum eine Analyse nach dem „COM-B“-Modell hilfreich sein.

Auch sind die Ergebnisse des qualitativen Studienteils (Mitarbeiter-Interviews) im Allgemeinen nicht dazu konzipiert, repräsentative Ergebnisse im Sinne der Generalisierbarkeit zu liefern. Ziel war es vielmehr, zugrundeliegende Ursachen der im Rahmen des quantitativen Studienteils ermittelten Defizite zu beleuchten.

\subsection{Versorgungslage}

Zur Realisierung des erklärten Projektziels der Optimierung der Versorgungsqualität rauchender Patienten war es zunächst erforderlich, die gegenwärtige Versorgungssituation zu ermitteln. Dies erfolgte mit Hilfe der Patientenbefragung in der ersten Studienphase und sollte als Ausgangs- und Bezugspunkt für die Evaluation der weiteren Interventionen und Prozessmodifikationen fungieren.

Die Auswertung jener Erhebung zeigte, dass ärztliche Entwöhnungsberatungen lediglich von $6,7 \%$, entsprechende Interventionen durch das pflegerische Personal von gerade einmal 1,3\% der hospitalisierten Raucher berichtet wurden. In keinem Fall erfolgte die Verordnung einer unterstützenden medikamentösen Therapie oder die Anbindung an die klinikinterne Entwöhnungsambulanz. Dementsprechend konnte in Phase I keine Erhöhung des Anteils derjenigen Raucher erzielt werden, die eine „hohe Bereitschaft zum Rauchverzicht“ äußerten. 
Zahlreiche andere Arbeiten zur Untersuchung der Interventionshäufigkeit bei Rauchern konnten ebenfalls eine optimierungsbedürftige Versorgungssituation aufzeigen, wenngleich sie nicht ganz so negativ ausfielen (Bartels et al. 2012, George et al. 2012, Goldstein et al. 1997). So befasste sich eine aktuelle Befragung aus den Vereinigten Staaten mit Rauchern, bei denen ein Bronchialkarzinom diagnostiziert wurde. Obwohl der wesentliche Risikofaktor in der überwiegenden Zahl der Fälle das Tabakrauchen ist, wurden nur $36 \%$ der Befragten hinsichtlich einer Entwöhnung beraten (Hildebrand und Sastry 2013). Eine andere Erhebung bei über 1600 hospitalisierten rauchenden Patienten mit akutem Myokardinfarkt zeigte, dass bei Entlassung aus der Klinik lediglich bei $14 \%$ eine Entwöhnungsmedikation verordnet wurde (Katz et al. 2011).

Eine Übersichtsarbeit zur Häufigkeit von Raucherentwöhnungsinterventionen in Kliniken lieferte in ihrer Metaanalyse unter Einschluss von 33 Studien folgende Ergebnisse: Die Erhebung des Rauchstatus erfolgte bei $60 \%$ der Patienten, wohingegen eine Beratung oder ein Ratschlag zum Rauchverzicht bei $42 \%$ unternommen wurde. Noch weniger Rauchern (14 \%) wurde eine Nikotinersatztherapie angeraten oder verschrieben und lediglich $12 \%$ wurden nachbetreut oder an eine weitere Entwöhnungsinstitution verwiesen (Freund et al. 2008).

Daten aus Deutschland gibt es vor allem aus dem Bereich der hausärztlichen Versorgung. Bei niedergelassenen Internisten und Allgemeinmedizinern bleibt in rund $25 \%$ der Fälle das Rauchen oder eine Tabakabhängigkeit unerkannt (Hoch et al. 2004). Bei bekanntem Rauchstatus erfolgt bei etwa der Hälfte der Patienten ein Ratschlag zum Rauchverzicht oder eine Beratung. Spezielle Entwöhnungsmaßnahmen wie verhaltenstherapeutische Interventionen oder der Einsatz von Medikamenten werden in 6,6 - 12,9 \% der Fälle initiiert (Hoch et al. 2004). Im stationären Bereich lieferte eine Befragung an 39 deutschen Kliniken mit pneumologischem Schwerpunkt vergleichsweise hohe Werte für ärztliche Entwöhnungsberatungen (Rupp et al. 2010). So berichteten rund drei Viertel der telefonisch befragten Pneumologen, bei 80$100 \%$ der hospitalisierten Raucher einen intensiveren Ratschlag zum Rauchverzicht zu erteilen. Der regelmäßige Einsatz von Entwöhnungsmedikamenten hingegen erfolgt in keiner der befragten Kliniken, regelmäßige Entwöhnungsinterventionen wie eine Verhaltenstherapie werden lediglich in drei Kliniken angeboten.

Die Zusammenschau dieser Ergebnisse ebenso wie die erhobenen Daten der vorliegenden Studie an der UMG implizieren einen dringenden Handlungsbedarf vor dem Hintergrund der in der Einleitung dargestellten leitliniengerechten Behandlung der Tabakabhängigkeit. Sämt- 
liche Studien, die diese defizitäre Versorgungslage herausstellen, betonen in ihren Schlussfolgerungen die Notwendigkeit von Interventionen und Vorkehrungen zur Verbesserung der Versorgungssituation.

Dabei verdeutlicht die im Rahmen dieses Projektes durchgeführte Erhebung (Patientenbefragung zu Beginn der Klinikaufnahme, Phase I) ein durchaus vorhandenes Potential für etwaige Maßnahmen: Etwa 70 \% der hospitalisierten Raucher hatten in der Vergangenheit schon einmal versucht, mit dem Rauchen aufzuhören, in der Mehrzahl der Fälle aus gesundheitlichen Gründen. Knapp $60 \%$ zeigten eine hohe Aufhörbereitschaft bei Aufnahme, darüber hinaus äußerten $40 \%$ einen expliziten Beratungswunsch bezüglich einer Tabakentwöhnung.

Dies deckt sich mit einer europaweiten Umfrage unter 8000 Patienten in allgemeinmedizinischer Behandlung, bei der rund $64 \%$ der Raucher der Ansicht war, ihr Rauchverhalten ändern zu müssen. Nahezu die Hälfte wünschte sich diesbezüglich konkrete Ratschläge von ihrem behandelnden Arzt (Brotons et al. 2011).

\subsection{Effektivität von Trainingsmaßnahmen}

Die vorliegende Interventionsstudie gründete auf der Annahme, dass sich die Versorgung rauchender Patienten durch eine Standardisierung der Abläufe auf den kardiologischen Stationen und insbesondere ein Mitarbeiter-Training verbessern ließe. Ein ähnliches Vorgehen wurde in verschiedenen anderen Studien gewählt, wobei wie in der vorliegenden Arbeit zumeist nur moderate Effekte beobachtet wurden.

Vor einer Diskussion möglicher Ursachen und Konsequenzen sollen an dieser Stelle einige prominente Vorstudien skizziert werden. Zu beachten ist hierbei, dass ein unmittelbarer Vergleich der Ergebnisse des vorliegenden Projektes mit denen anderer Autoren in vielen Fällen nicht uneingeschränkt möglich ist. So gibt es beispielsweise große studienimmanente Unterschiede bei den jeweils zur Verfügung gestellten Ressourcen sowie bei dem Umfang und der Intensität der im Rahmen der Raucherentwöhnung durchgeführten Maßnahmen (Rigotti et al. 2008). Das Spektrum reicht dabei von einem kurzen Ratschlag zum Rauchverzicht bis hin zu mehrmaligen und intensiven Kontakten mit verhaltenstherapeutisch und psychologisch geschulten Kräften in Kombination mit interdisziplinär zusammengestellten Entwöhnungsmedikamenten und einer umfassenden Nachbetreuung.

Auch das Probandenkollektiv spielt eine gewisse Rolle. In einer systematischen Übersichtsarbeit konnte gezeigt werden, dass die Aufnahmediagnose der Patienten einen, wenn auch nur 
geringen, Einfluss auf die ermittelte Abstinenzrate hat. So liegt der Effekt einer intensiven Beratung bei aufgrund einer kardiovaskulären Erkrankung hospitalisierten Raucher etwas höher (OR: 1,81; 95 \% - KI: 1,53 - 2,15) im Vergleich zu Rauchern aus Studien, bei denen Probanden mit sämtlichen Diagnosen eingeschlossen wurden (OR: 1,43; $95 \%$ - KI: 1,17 - 1,75) (Rigotti et al. 2008).

Einschränkungen hinsichtlich der Vergleichbarkeit ergeben sich letztlich auch dadurch, dass bei der Mehrzahl der publizierten Studien zur Untersuchung von Tabakentwöhnungsinterventionen im stationären Bereich der Erfolg der implementierten Maßnahmen anhand der Abstinenzrate oder der Abstinenzdauer der Studienteilnehmer evaluiert wurde. Entsprechend orientieren sich auch die auf diesem Gebiet publizierten Übersichtsarbeiten (Rigotti et al. 2012) an diesen Endpunkten, die in der vorliegenden Arbeit allerdings nicht gewählt wurden.

In einer in der Schweiz durchgeführten Studie wurden in den allgemein-internistischen Abteilungen zweier Universitätskliniken ärztliche Mitarbeiter mit Hilfe von zwei halbtägigen Schulungen hinsichtlich einer Tabakentwöhnungsbehandlung trainiert (Cornuz et al. 2002). Die Schulungsmaßnahme bestand aus Videoanalysen von Beratungsgesprächen, der Vermittlung theoretischer Kenntnisse anhand von Fallbeispielen, Workshops, Rollenspielen sowie der Durchführung von Beratungen an Schauspielpatienten.

Bei dem Vergleich der Interventionsgruppe (rauchende Patienten, deren behandelnde Ärzte an der speziellen Trainingsmaßnahme teilnahmen) mit der usual-care-Gruppe zeigte sich neben einer erhöhten Abstinenzrate der Raucher nach einem Jahr (13\% vs. $5 \%$; p = 0,005) vor allem eine höhere Qualität der ärztlichen Beratungen, gemessen an den im Folgenden auszugsweise aufgeführten, ärztlicherseits angewandten Aktivitäten, die von den beratenen Rauchern berichtet wurden: Erhebung des Rauchstatus (87\% vs. $78 \%$ ), expliziter Ratschlag zum Rauchverzicht (39 \% vs. $29 \%$ ), Anbieten von Hilfe (23\% vs. $7 \%$ ), Erörterung von Strategien zur Rückfallprophylaxe (15\% vs. $6 \%$ ) und Aushändigen von Informationsmaterialien (14\% vs. $1 \%)$.

Auch hier zeigte sich insgesamt betrachtet eine eher moderate Verbesserung der Versorgungssituation. Zu berücksichtigen ist dabei, dass als alleinige Maßnahme lediglich eine Schulung der Ärzte stattfand. Prozessoptimierungen oder systematische Änderungen der vorhandenen Stationsabläufe, also den Aspekt der Gelegenheit betreffende Interventionen wie etwa die Bereitstellung von Entwöhnungsmedikamenten, die Implementierung eines standardisierten Aufnahmeprocederes oder übergeordneter Richtlinien, wurden hingegen nicht vorgenommen. 
So sollte konformes Verhalten durch Prozessmodifikationen wie Erinnerungen an durchzuführende Beratungen ebenso wenig gefördert werden wie durch das Wissen der auf Station eingesetzten Mitarbeiter um die Ziele und Details der Studie, was mittels einer Verblindung ärztlicherseits erreicht wurde. So wurden die Ärztinnen und Ärzte nicht über die Patientenbefragungen zum Thema Raucherentwöhnung in Kenntnis gesetzt. Die Beratungen sollten von den ärztlichen Mitarbeitern alleine auf der Grundlage vermittelter Kenntnisse und Fertigkeiten bezüglich der Raucherentwöhnung initiiert werden.

Die von den Autoren der Studie herausgestellte Verbesserung der Beratungsqualität lässt sich vor allem auf die sehr praxisbezogenen und interaktiven Bestandteile der Trainingsmaßnahme zurückführen (Cornuz et al. 2002). Das aktive Erlernen bestimmter Gesprächstechniken in Abhängigkeit von dem Stadium der Verhaltensveränderung des Rauchers, angelehnt an das Transtheoretische Modell der Verhaltensänderung (DiClemente et al. 1991, Prochaska und Velicer 1997), stand hier im Vordergrund und war der ausschlaggebende Faktor der verbesserten Versorgungsqualität.

Angewendet auf das „COM-B“-Modell wurden auf diese Weise allerding nur die Fertigkeiten als eine der drei wichtigen Determinanten des Verhaltens beeinflusst. Es wäre zu erwarten, dass gleichzeitige Modifikationen der Prozessabläufe und der Ressourcen auf Station (Aspekt der Gelegenheit) ebenso wie eine Einbindung der Pflegekräfte in die Schulung und somit in die Entwöhnungsbehandlung ein noch erfolgreicheres Outcome zur Folge hätte.

Solche dort nicht angewandten strukturellen Maßnahmen waren hingegen ein entscheidender Bestandteil der in dieser Arbeit dargestellten Studie an der UMG, wohingegen die Trainingsmaßnahme in Göttingen deutlich weniger interaktiv und praxisbezogen gestaltet war. Entsprechend sollten die Erfolge und Erfahrungen derartiger praktisch orientierter Schulungen, wie sie Cornuz in seiner Arbeit herausstellen konnte, Eingang in zukünftige Trainingsmaßnahmen finden.

Im Rahmen einer 2013 im British Medical Journal veröffentlichten Arbeit wurde der Fokus bei der Untersuchung von Tabakentwöhnungsmaßnahmen bei hospitalisierten Rauchern im Gegensatz zu der zuvor vorgestellten Studie vordergründig auf die Implementierung strukturierter Abläufe gelegt (Murray et al. 2013).

Auf den entsprechenden Interventionsstationen einer Universitätsklinik wurden Raucher systematisch identifiziert. Im Anschluss an einen generellen Ratschlag zum Rauchverzicht sowie nach Aushändigung von Informationsmaterialien sollten bei allen entwöhnungswilligen Patienten weitere Maßnahmen eingeleitet werden. Diese umfassten ausführlichere Beratungsge- 
spräche, das Ansetzen einer begleitenden Entwöhnungsmedikation und die fakultative poststationäre Anbindung an eine Entwöhnungsinstitution. Die Anzahl der insgesamt eingeschlossenen Raucher betrug fast 500. Als primärer Endpunkt wurde auch hier die Abstinenzrate definiert, wesentlicher für einen Vergleich mit der in dieser Arbeit vorgestellten Studie sind allerdings die sekundären Endpunkte.

Demnach führten die implementierten Handlungsrichtlinien und Abläufe zu einer verbesserten Versorgungssituation der Raucher, nachfolgend dargestellt durch den Vergleich der Interventionshäufigkeiten innerhalb der Interventionsgruppe mit denen der usual-care-Gruppe: Kurzer Ratschlag zum Rauchverzicht mit Anbieten verhaltenstherapeutischer Unterstiutzung und/oder einer Pharmakotherapie (100\% vs. $46 \%)$, Durchführung ausführlicher verhaltenstherapeutischer Entwöhnungsberatungen (70 \% vs. $29 \%$; OR: 5,71; 95 \% - KI: 3,58 9,09; p < 0,001), Verordnung einer Entwöhnungsmedikation (49 \% vs. 27 \%; OR: 3,95; 95\% KI: $1,81-8,63 ; \mathrm{p}<0,001)$ sowie Anbindung an eine Tabakentwöhnungseinrichtung nach der Klinikentlassung (55 \% vs. $6 \%$; OR: 21,8; $95 \%$ - KI: 9,4 - 50,6; p < 0,001).

Diese Ergebnisse zeigen einen ausgeprägteren Effekt auf die Versorgungssituation der rauchenden Patienten. Entscheidend bei der Interpretation dieser Resultate ist, dass zum einen die initialen Maßnahmen wie die Identifikation der Raucher sowie der erste kurze Ratschlag zum Rauchverzicht von dem Studienpersonal, zum anderen die nachfolgenden intensiveren Interventionen jeweils von speziell ausgebildetem Personal durchgeführt wurden. Letztere wiesen fundierte Kenntnisse bei der Tabakentwöhnungsbehandlung auf, insbesondere hinsichtlich verhaltenstherapeutischer Maßnahmen wie der motivierenden Gesprächsführung. Angesetzt wurden die Erstgespräche auf eine Dauer von etwa 20 - 30 Minuten, alle weiteren Konsultationen auf bis zu 10 Minuten. Insgesamt fielen bis auf die Autorisierung eines eventuellen Einsatzes einer Entwöhnungsmedikation keine zusätzlichen Aufgaben für das ärztliche und pflegerische Stammpersonal an.

Auf diese Weise konnten gewisse Hindernisse von vorneherein vermieden werden, hätte man die im Zuge der implementierten Maßnahmen zusätzlich anfallenden Aufgaben gänzlich oder teilweise auf das Stammpersonal übertragen (Murray et al. 2013). Solche Barrieren wie ungenügende Fertigkeiten und Kenntnisse bei der Entwöhnungsbehandlung oder eine mangelhafte Rollenkongruenz bzw. Motivation konnten bei dem Projekt an der UMG als wesentliche Limitationen identifiziert werden, bei dem sämtliche Entwöhnungsaktivitäten von der regulären Belegschaft übernommen wurden. Dies vermag zu einem gewissen Teil den größeren Erfolg des Projektes von Murray zu erklären. Berücksichtigt werden muss in dieser Hinsicht jedoch 
der erheblich höhere finanzielle Aufwand durch die Einstellung dieses speziell geschulten Personals.

Dennoch sind die daraus gewonnenen Erkenntnisse ein allgemeines Plädoyer für organisierte und strukturierte Abläufe bei der Versorgung hospitalisierter Raucher sowie für eine Beteiligung und Einbindung professioneller Kräfte.

In diesem Zusammenhang soll abschließend das sogenannte Ottawa-Modell vorgestellt werden, welches sich bezüglich der getroffenen Interventionen und Maßnahmen im Gegensatz zu den vorherigen Studien nicht nur auf eine der drei Verhaltensdeterminanten aus dem „COM-

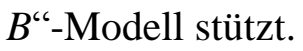

In einem äußert umfassenden Rahmen wurden an dem University of Ottawa Heart Institute (UOHI) durch eine Reihe von Prozessmodifikationen die strukturellen Rahmenbedingungen für eine leitlinienorientierte Raucherentwöhnungsbehandlung bei KHK-Patienten geschaffen. Seit seiner Entwicklung wurde das Konzept in mehreren anderen Kliniken in Kanada erfolgreich eingeführt und evaluiert (Reid et al. 2010, Reid et al. 2006). Unterstützt durch die Bereitstellung von finanziellen Mitteln in Höhe von $\$ 144.000$ jährlich werden dort folgende Maßnahmen durchgeführt: Zunächst die systematische Identifikation von Rauchern beim Aufnahmegespräch mit anschließendem, kurzen Ratschlag zum Rauchverzicht und Dokumentation dessen. Im weiteren Verlauf erfolgt eine an die individuelle Aufhörbereitschaft des Patienten adaptierte, ausführlichere Beratung durch eine speziell dafür eingesetzte und ausgebildete Pflegekraft. Zuvor bereitgestellte Entwöhnungsmedikamente werden in Absprache mit den behandelnden Ärzten verordnet und Selbsthilfematerial einschließlich weiterer Kontaktadressen zusammen mit einem Informationsblatt speziell zur Pharmakotherapie ausgehändigt. Die Nachbetreuung der Raucher wird durch ein automatisches, interaktives Anruf-System gewährleistet, welches die Patienten in gewissen Abständen kontaktiert und auf diese Weise eventuelle Rückfalltendenzen oder eine Wiederaufnahme des Rauchens erkennt. In solchen Fällen können weitere Telefonkontakte, die Vermittlung an eine Entwöhnungshotline oder an örtliche Entwöhnungsinstitute initiiert werden. Regelmäßige Trainingsmaßnahmen der auf den Stationen eingesetzten ärztlichen und pflegerischen Mitarbeiter sollen die Qualität der Versorgung nachhaltig sichern.

Die Resultate im Anschluss an eine einjährige Erhebungsphase unmittelbar nach der Implementierung sämtlicher Maßnahmen am UOHI zeigten, dass $91 \%$ der hospitalisierten Raucher von der professionellen Kraft beraten wurden, davon fast ein Drittel in einem intensiveren Zeitrahmen. Die häufigsten Gründe für eine fehlende Beratung waren eine zu kurze Liege- 
dauer oder eine strikte Ablehnung jeglicher Unterstützungsangebote. Nikotinersatzmittel wurden in $33 \%$ der Fälle verschrieben, $53 \%$ erhielten zusätzliche Entwöhnungsinterventionen nach der Entlassung. Davon entschieden sich $90 \%$ für Telefongespräche mit der Entwöhnungskraft, während $10 \%$ an einem außerklinischen Entwöhnungsprogramm teilnahmen (Reid et al. 2006).

Das Konzept des Ottawa-Modells wurde kurze Zeit später in neun weiteren Kliniken im Südosten Kanadas implementiert. Die nachfolgende Evaluation dieser Projektausweitung lieferte für die dort behandelten Raucher mittels eines Vorher-Nachher-Vergleichs eine höhere kontinuierliche 6-Monats-Abstinenzrate als vor der Einführung des Konzepts an den jeweiligen Kliniken (29,4 \% vs. 18,3\%; OR: 1,71; 95\% - KI: $1,11-2,64 ; \mathrm{Z}=2,43 ; \mathrm{I}(2)=0 \% ; \mathrm{p}=0,02)$ (Reid et al. 2010).

Das Ottawa-Modell vereint alle zuvor diskutierten Punkte. An den Leitlinien orientiert sind von der systematischen Identifikation der Raucher bis hin zur Sicherstellung einer Nachbetreuung und Rückfallprophylaxe alle Abläufe strukturiert und organisiert. Der Einsatz von speziell ausgebildeten Fachkräften ist ebenso gewährleistet wie die Einbeziehung der Stammbelegschaft, regelmäßige Trainingsmaßnahmen eingeschlossen.

Besonders hervorgehoben werden sollen einige Elemente, in denen sich das kanadische Model von der SOP der UMG unterscheidet und die möglicherweise eine Erklärung für den höheren Erfolg bzw. Anregungen für Verbesserungen liefern können:

So geht es zum einen um das grundsätzliche Procedere bei all jenen Patienten, die lediglich eine geringe oder gar eine fehlende Bereitschaft zum Rauchverzicht erkennen lassen. Im Rahmen der Mitarbeiterbefragung an der UMG konnte als eine Barriere identifiziert werden, dass sich Mitarbeiter von einem mangelnden Interesse, einem fehlenden Nachfragen oder im extremsten Fall von einer ablehnenden Haltung seitens des Rauchers hinsichtlich weiterer Entwöhnungsaktivitäten demotivieren ließen. Dazu eine Pflegekraft: „Die meisten sagen: Nein, ich möchte weiterrauchen und dann hakt man natürlich auch nicht weiter nach. Man möchte ja auch nicht aufdringlich sein“. Der Grundsatz bzw. das Vorgehen in Ottawa hingegen zielt darauf, auch bei diesen Patienten das Gespräch zu suchen, wobei die Schwerpunkte dann auf der Erörterung der Vor- und Nachteile eines fortgesetzten Tabakkonsums liegen. Diese Herangehensweise der beratenden Mitarbeiter sollte entsprechend gefördert werden. So könnte beispielsweise ein motivierendes Gespräch nach dem „5R-Schema“ (Fiore et al. 2008) durchgeführt werden sowie im Anschluss weitere Beratungen angeboten werden, sofern Letzteres von dem Patienten gewünscht wird. 
Ebenfalls Einfluss auf die Motivation der Mitarbeiter mag die in Kanada praktizierte, umfassende Sicherstellung einer Nachbetreuung der Raucher haben. So zweifelten einige Pflegekräfte in Göttingen an der Effektivität der stationären Entwöhnungsmaßnahmen und bemerkten, dass viele Raucher den Tabakkonsum direkt nach der Entlassung wieder aufnehmen würden: „Es ist nicht so effektiv, weil die Leute... also die meisten rauchen weiter. Und schaffen es nicht, wenn sie dann entlassen sind“. Diese negative Erwartung wirkt sich nachteilig auf die Motivation aus, entsprechende Beratungen und Anstrengungen in Richtung Tabakentwöhnung zu unternehmen. Das Wissen um eine adäquate Weiterbehandlung ist in dieser Hinsicht sicherlich förderlich.

An dieser Stelle soll außerdem angemerkt werden, dass ein wesentlicher Bestandteil der am UOHI durchgeführten Beratungen die Einbeziehung der Angehörigen der Patienten ist, im Sinne eines ganzheitlichen Ansatzes. Passend dazu konnte eine Studie aus Deutschland zeigen, dass der Rauchstatus des Partners einen entscheidenden Einfluss auf die Motivation zum Rauchverzicht hat (Ruge et al. 2008). Diese ist geringer, sobald der jeweilige Partner selbst aktiver Raucher ist. Vor diesem Hintergrund sieht das Ottawa-Modell auch Beratungsangebote an rauchende Angehörige sowie gemeinsame Familiensitzungen vor.

Letztlich dürfte auch das Wissen um die Tatsache, dass sich das Konzept aus Ottawa als durchaus erfolgreich erwies und mittlerweile auch an weiteren Kliniken Einzug fand, die Motivation der beteiligten Mitarbeiter weiter steigern. In diese Richtung geht die Äußerung eines ärztlichen Mitarbeiters der UMG, dass sich die Motivation einiger Kollegen noch weiter steigern ließe, wüsste man um die Erfolge der durchgeführten Maßnahmen.

Bei allen positiven Aspekten muss dennoch berücksichtigt werden, dass das Ottawa-Modell in erheblicherem Maße materiell und finanziell gefördert wurde. So wurde unter anderem auch eine administrative Vollzeitkraft eingestellt. Fast noch bedeutender ist eine weitere Form der Unterstützung: Von der Ebene der Klinikleitung wurde das Vorhaben gestützt und gefördert mit dem Ziel, das Rauchen unter den hospitalisierten Patienten zu verringern. Dabei wurde der Rauchstatus nach Entlassung als Qualitätsindikator für die Leistung und Arbeit der Klinik definiert (Reid et al. 2006). Die Verpflichtung zu diesem Ziel vermag ein Ansporn zu weiteren Maßnahmen und Anstrengungen zu sein.

Bei der zu Beginn dieses Kapitels vorgestellten Studie aus der Schweiz wurde im Vergleich zu dem Modell aus Ottawa zur Optimierung der defizitären Versorgungssituation als vordergründige Interventionsmaßnahme eine Schulung ärztlicher Mitarbeiter vorgenommen (Cornuz et al. 2002). Mit der dadurch intendierten Beeinflussung der Fertigkeiten beschränkte man 
sich gemäß des „COM-B“-Modells auf lediglich einen der drei verhaltensdeterminierenden Aspekte. Somit vermag der Erfolg des Ottawa-Modells darin begründet zu liegen, dass im Zuge der dortigen Maßnahmen auch Einfluss auf die beiden anderen Determinanten (Gelegenheit, Motivation) genommen wurde. Im folgenden Kapitel sollen auf der Grundlage des „COM-B“-Modells systematisch einige konkrete Interventions-Optionen erarbeitet werden, mit denen die in dieser Studie identifizierten Defizite an der UMG behoben werden könnten.

\subsection{Systematische und theoretisch fundierte Herangehensweise mit Hilfe des Behaviour Change Wheel}

Die vorangegangene Darstellung ähnlicher Studien und Modelle kann unter Hervorhebung der jeweiligen Stärken und Schwächen gewisse Ansatzpunkte für die notwendigen Verbesserungen der an der UMG implementierten Versorgungsstrukturen liefern, wenngleich nicht alle Erkenntnisse ohne weiteres und uneingeschränkt übertragbar sind. Die weitere Analyse zum Zweck der Ableitung konkreter Empfehlungen und Interventionen soll, theoretisch begründet und in einem strukturierterem Rahmen, anhand des sogenannten Behaviour Change Wheel $(B C W)$ stattfinden.

Abb. 4: : Behaviour Change Wheel (Michie et al. 2011)
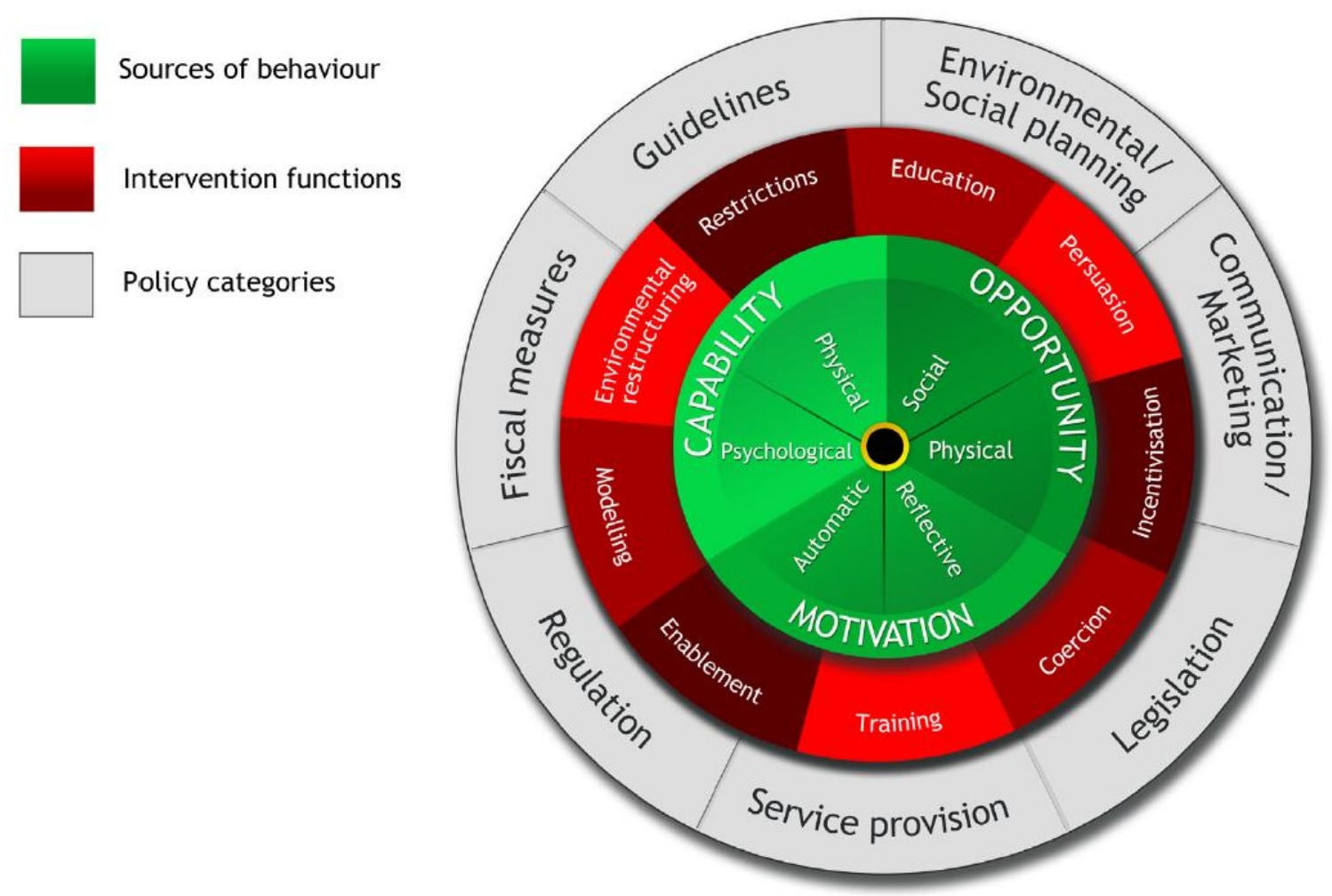
Das $B C W$ wurde unter Berücksichtigung der bereits vorhandenen, zumeist allerdings unvollständigen und inkohärenten Modelle entwickelt, um systematisch Interventionen und Richtlinien abzuleiten, die eine Änderung des Verhaltens in Richtung des gewünschten Verhaltens induzieren (Michie et al. 2011). Im Zentrum des Modells stehen dabei die Hauptdeterminanten des Verhaltens im Sinne des bereits vorgestellten „COM-B“"-Modells (siehe Kapitel 1.7), welche sich durch bestimmte Interventionen (intervention functions, in Abb. 4 rot gekennzeichnet) beeinflussen lassen.

Die Interventionsfunktionen wiederum werden unterstützt bzw. ermöglicht durch gewisse übergeordnete Grundsätze und Richtlinien (policy categories, in Abb. 4 grau dargestellt). So ergibt sich von innen nach außen der charakteristische Aufbau des $B C W$, welches alle relevanten Elemente und Maßnahmen für geplante Änderungen eines Verhaltens abbildet (siehe Abb. 4).

Tab. 9: Verknüpfung der Elemente des „COM-B“-Modells mit den jeweils zur Verfügung stehenden Interventionsfunktionen; modifiziert nach Michie et al. 2011

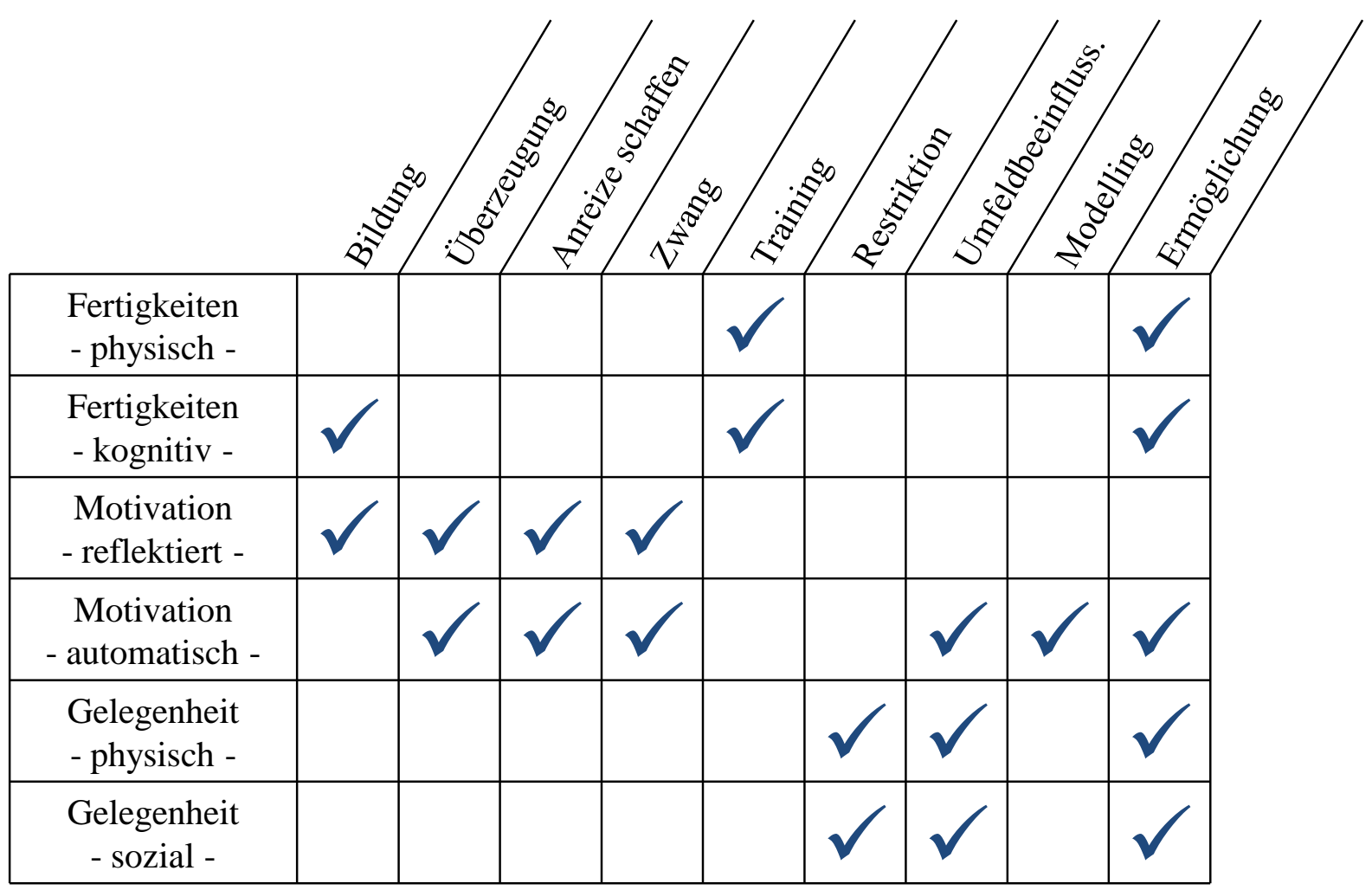

Ergänzend dazu liefert Tab. 9 eine Zuordnung der entsprechend zur Verfügung stehenden Interventionsfunktionen zu den jeweiligen Verhaltensdeterminanten, die es zu beeinflussen gilt. Die weitere Darstellung soll sich an diesem Modell orientieren. 
Schon an dieser Stelle soll angemerkt werden, dass nicht zu jedem der sechs Verhaltensaspekte sämtliche in der Tabelle vorgeschlagenen Interventionsfunktionen als sinnvolle und praktikable Optimierungsmaßnahme in Betracht kommen.

\subsubsection{Motivation}

Im Rahmen der Auswertung des qualitativen Studienteils (Mitarbeiter-Interviews) konnten wesentliche Barrieren insbesondere im Bereich der reflektierten Motivation nachgewiesen werden. Unter anderem zeigte sich, dass eine Mehrheit der befragten Pflegemitarbeiter die Ansicht vertrat, die Thematisierung des Rauchens sei eine primär ärztliche Aufgabe. Diese mangelhafte Rollenkongruenz stellte neben der selektiven Durchführung von Entwöhnungsberatungen eine der Hauptursachen für den kaum vorhandenen Anstieg der Interventionshäufigkeit dar.

Gemäß der oben aufgeführten Tabelle (Tab. 9) kommen vier verschiedene Interventionsfunktionen infrage, Defiziten im Bereich der reflektierten Motivation zu begegnen: Bildung, Überzeugung, Zwang sowie der Einsatz von Anreizen. Im Anschluss werden einige Beispiele für Interventionen genannt, die sich auf diese vier Bereiche beziehen:

- Bildung: Ein besseres Verständnis der Hintergründe der SOP kann die Motivation erhöhen, die Empfehlungen der SOP umzusetzen. Entsprechend könnten im Zuge einer erneuten Fortbildungsveranstaltung die Inhalte der SOP sowie die spezifische Aufgabenverteilung rekapituliert werden unter Hervorhebung der Rolle der Pflegekräfte, die durch die Identifizierung der Raucher sowie einen ersten Ratschlag zum Rauchverzicht einschließlich des Anbietens von Hilfe einen äußerst wichtigen Beitrag bei der Raucherentwöhnung leisten. Dies muss den pflegerischen Mitarbeitern nachhaltig vermittelt werden.

- Überzeugung: Die reflektierte Motivation der Mitarbeiter/innen zur Umsetzung der SOPEmpfehlungen wäre möglicherweise höher, wenn es gelänge, insbesondere die Pflegekräfte von der Effektivität der Maßnahme zu überzeugen. Dies kann beispielsweise durch den expliziten Verweis auf wissenschaftliche Studien versucht werden. Eine Vielzahl von Arbeiten konnte die Effektivität einer kurzen Entwöhnungsintervention durch pflegerische Kräfte herausstellen (Chouinard und Robichaud-Ekstrand 2005, Gies et al. 2008, Katz et al. 2012, Rice et al. 2013). Bestätigt wird dies durch die Übersichtsarbeit der CochraneCollaboration (Rice et al. 2013). Den Pflegekräften sollte verdeutlicht werden, dass ihre 
Rolle bei der Tabakentwöhnungsbehandlung ebenso entscheidend und erfolgversprechend ist wie die der ärztlichen Mitarbeiter.

- Zwang: Das gewünschte Verhalten ließe sich nötigenfalls auch durch die Einführung einer impliziten oder expliziten Verpflichtung zur Ausübung der jeweiligen rollenspezifischen Aufgaben induzieren. Da die meisten pflegerischen Aufnahmegespräche unter Verwendung der stationären Aufnahmebogen erfolgen, könnten diese ergänzt werden durch einige zusätzliche Fragen zum Rauchen sowie der Aufhörmotivation. So könnte die auf diesem Bogen aufgeführte alleinige Frage nach dem Rauchstatus unter dem Absatz der kardiovaskulären Risikofaktoren erweitert werden um Fragen nach der Anzahl der Raucherjahre, der Menge der täglich konsumierten Zigaretten, der Aufhörmotivation und dem Wunsch nach einer Unterstützung. Bei der Beantwortung dieser Fragen kommt es zwangsläufig zum Thematisieren des Rauchens und die Pflegekraft wird außerdem an die geforderte Intervention erinnert. Eine eventuelle Kontrolle der Erhebung dieser Punkte könnte durch die ärztlichen Mitarbeiter erfolgen, die im Rahmen der weiteren Patientenbehandlung meist auf den Aufnahmebogen zurückgreifen.

Alternativ oder zusätzlich könnte gefordert werden, eine erfolgte Beratung in den Patientenunterlagen oder an der Wandtafel im Stationszimmer zu dokumentieren. Ob diese Maßnahmen letztendlich auch in einem patientenadaptierten Ratschlag zum Rauchverzicht seitens der Pflegekräfte münden, müsste im Rahmen einer weiteren Studie untersucht werden.

- Anreiz: Belohnungsmaßnahmen können sich auf die reflektierte Motivation auswirken; es ist aber anzunehmen, dass ihre Wirkung auf die automatische Motivation stärker ist. Daher wird dieser Aspekt gemeinsam für die reflektierte und die automatische Motivation im folgenden Absatz diskutiert.

Bezüglich der automatischen Motivation liefert das Verhaltensmodell (siehe Tab. 9) die potenziellen Interventionen Überzeugung, Anreiz, Zwang, Umfeldbeeinflussung, Modelling und Ermöglichung.

Zunächst soll jedoch auf die festgestellte Diskrepanz bei der Wahrnehmung und Bewertung der Entwöhnungsberatungen eingegangen werden: Gemäß der Mitarbeiterbefragung beobachteten 40,9\% der befragten Pflegekräfte eine ablehnende Haltung der Raucher in Bezug auf die durchgeführten Beratungsgespräche. Dies wurde ebenfalls im Rahmen der Mitarbeiterinterviews geäußert. Im Gegensatz dazu bewerteten die meisten Raucher die Gespräche positiv (Phase I: 83,3 \%; Phase II: 83,9 \%). 
Eine potenzielle Erklärung dafür könnte eine falsche Wahrnehmung seitens der Pflegekräfte sein, wonach eine eventuell vorhandene initiale Skepsis, ein fehlender Enthusiasmus oder ein mangelndes Interesse des Rauchers an der Beratung als Ablehnung interpretiert wurde. Dabei könnte auch die sogenannte selbsterfüllende Prophezeiung (self-fulfilling prophecy) eine Rolle spielen. Demnach würden die pflegerischen Mitarbeiter die Reaktionen der Patienten besonders negativ wahrnehmen, weil sie schon mit der Erwartung in das Gespräch gegangen sind, dass sie abgelehnt würden.

Eine andere Erklärung für die beobachtete Diskrepanz in der Wahrnehmung berücksichtigt, dass die Beratungen überwiegend von den Ärzten durchgeführt wurden. Folglich könnte sich die mehrheitlich positive Bewertung der Patienten vor allem auf die ärztlichen Interventionen beziehen. Die von Pflegekräften beobachtete ablehnende Haltung mag dann in den oftmals vor den Arztkontakten stattfindenden Aufnahmegesprächen vernommen worden sein, wo der Rauchstatus erhoben und ein erster Ratschlag zum Rauchverzicht erteilt werden sollte. Die beiden unterschiedlichen Bewertungen schließen sich also nicht unbedingt aus.

Als denkbare Ursache für die Ablehnung kann eine mangelnde Empathie oder ein geringes Fingerspitzengefühl bei den Erstberatungen in Erwägung gezogen werden, wonach die gerade auf Station eintreffenden, teilweise aufgrund schwerwiegender Erkrankungen hospitalisierten Patienten direkt mit Fragen bezüglich des Rauchverhaltens und der Aufhörmotivation überrumpelt wurden. Ebenfalls könnte der Punkt „Rauchen“ von den Pflegekräften nüchtern und stakkatoartig neben der Frage nach Allergien abgehakt worden sein, nach dem Prinzip: „Rauchen Sie?“ - „Ja.“ „Wollen Sie aufhören?“ - „Nun...“ „Es gibt Medikamente und eine Entwöhnungsambulanz.“ - „Nein danke.“ Dies könnte ein ablehnendes Verhalten des Patienten hervorgerufen haben.

Vor diesem Hintergrund wäre es im Rahmen einer weiteren Studie sinnvoll, den Ablauf und Charakter der pflegerischen Erstkonsultationen genauer zu untersuchen, beispielsweise indem die jeweiligen Aufnahmegespräche durch eine unabhängige Person beobachtet würden. Denkbar wäre ebenfalls eine Videoaufzeichnung der Beratungen unter der Voraussetzung eines beiderseitigen Einverständnisses.

Passend zu diesem Aspekt gibt es eine Arbeit, in der die Wahrnehmung des ärztlichen Ratschlags zum Rauchverzicht bzw. das Thematisieren des Rauchens im Rahmen von semistrukturierten Interviews mit Patienten untersucht wurde (Butler et al. 1998). Kritisiert wurde von den Patienten unter anderem, dass die oft angesprochenen negativen Auswirkungen des Rauchens hinlänglich bekannt seien und die Ratschläge oft zu „rituell“, standardisiert und „wenig 
individuell“" seien. Die unmittelbaren Reaktionen der Raucher darauf reichten von Verärgerung, Schuldgefühlen und dem Ignorieren des Gesagten bis hin zu einem Arztwechsel. Dabei wünschten sich die Raucher in Bezug auf die Gespräche einen respektvollen Umgang ohne Bevormundung und eine patientenadaptierte Beratung mit Rücksicht auf die individuelle Aufnahmebereitschaft (Butler et al. 1998).

An dieser Stelle sollen nun einige Beispiele für die eingangs aufgeführten Interventionsfunktionen benannt werden:

- Überzeugung: Durch positive Erfahrungen können Mitarbeiter/innen möglicherweise auch ohne kognitive Reflexion von der Sinnhaftigkeit einer strukturierten Raucherberatung überzeugt werden. Eine solche Überzeugung kann sich positiv auf die automatische Motivation auswirken. Mit Hilfe von Gesprächen und Rollenspielen, in denen die medizinischen Mitarbeiter den Platz der beratenen Raucher einnehmen, könnten diese selbst erfahren, welche Wahrnehmungen und Gefühle sich bei ihnen in Bezug auf bestimmte Gesprächstechniken und Herangehensweisen des beratenden Gegenübers einstellen.

- Anreiz: Wie oben geschildert, wirken sich Belohnungen sowohl auf die reflektierte als auch auf die automatische Motivation aus. Vorstellbar wäre die Implementierung eines Systems der Evaluation, wonach die beratenen Raucher ihrer Zufriedenheit oder ihrem Unmut über die erfolgten Beratungsgespräche Ausdruck verleihen können. Auf diese Weise erhielte jeder Mitarbeiter ein unmittelbares Feedback, wie sein Engagement und seine Entwöhnungsaktivitäten von den Rauchern aufgenommen würden und wo die jeweiligen Stärken und Schwächen liegen. Anerkennende und lobende Worte der Patienten vermögen einen großen Ansporn zu weiteren Aktivitäten darzustellen und somit die Motivation entscheidend zu fördern. Außerdem könnte so die Fehlwahrnehmung der Pflegekräfte korrigiert werden, dass ihre Beratungen von den Patienten nicht gewünscht seien. Unter Umständen kann auch von einer negativen Bewertung oder der Bemängelung einer nicht durchgeführten, aber vom Raucher erwünschten Beratung eine motivationssteigernde Wirkung ausgehen. Hierbei spielt die Form des Feedbacks aber eine wichtige Rolle. Anerkennung und Lob als Anreiz zu weiterem Engagement auf dem Gebiet der Tabakentwöhnungsberatung kann selbstverständlich auch oder bestenfalls ergänzend zu dem vorgeschlagenen Patientenfeedback durch die leitenden Mitarbeiter der Station kommuniziert werden, also der Ebenen- bzw. Stationsleitung, den Oberärzten oder dem Chefarzt. Darüber hinaus wäre es möglich, besonders engagierte Mitarbeiter oder auch ganze Stationen für ihre Arbeit und ihren Einsatz hinsichtlich der Versorgung rauchender Patienten 
auszuzeichnen. Dies setzt eine sorgfältige Dokumentation und Evaluation entsprechender Aktivitäten voraus, was wiederum zu einem gewissen Teil mit Hilfe des zu Beginn dieses Abschnittes vorgeschlagenen Bewertungssystems realisiert werden könnte und außerdem zur Qualitätssicherung der SOP beitragen würde.

- Zwang: Durch eine Überprüfung und Kontrolle der durchzuführenden Entwöhnungsberatungen ließe sich ein gewisser Zwang auf die Mitarbeiter ausüben. Im Rahmen der Übergabebesprechungen könnten die beteiligten Schicht- oder Stationsleiter unter Zuhilfenahme der Stationstafel, an der die jeweiligen Raucher markiert sind, die noch erforderlichen Beratungsinterventionen identifizieren und diese gegebenenfalls direkt an die zuständige Pflegekraft delegieren. Eine solche Kontrollfunktion könnte ebenso durch einen Entwöhnungsbeauftragten ausgeübt werden, dessen Einsatz in Kapitel 4.5.3 (Aspekt der Gelegenheit) thematisiert wird.

- Umfeldbeeinflussung: In diese Kategorie fallen Modifikationen des Umfeldes, die bei den Mitarbeitern eine (unbewusste) Steigerung der Motivation induzieren. So könnte in Anlehnung an den Aspekt des vorigen Absatzes für die Pflegekräfte deutlicher gekennzeichnet werden, bei welchen Patienten ein entsprechender Interventionsbedarf besteht. Anstelle der Markierung der Raucher an der Stationstafel mit einem Kreuz, was in einigen Fällen möglicherweise übersehen wurde, sollte eine etwas auffälligere Kennzeichnung gewählt werden. In diesem Fall bietet sich eine Art „Ampel“ an mit den Farben rot oder grün (jeweils für „noch nicht beraten“ oder „Beratung erfolgte“), die den Bedarf einer Entwöhnungsintervention in einer deutlich sichtbareren Weise darstellt.

Als weitere umfeldbeeinflussende Maßnahme kämen diverse Aushänge zum Thema Rauchen in Betracht. In Form von Postern oder Plakaten ließen sich Informationen zum Tabakrauchen, zum „rauchfreien Krankenhaus“ und zu den verfügbaren Behandlungsmöglichkeiten allgemein und speziell an der UMG veranschaulichen. Empfehlenswert wäre vor allem die Vorstellung der klinikeigenen Entwöhnungsambulanz. Auf den Stationsfluren ausgehängt, wären sie für Patienten und Mitarbeiter gleichermaßen sichtbar und würden die Relevanz der Tabakabhängigkeit unterstreichen.

Daneben könnten spezielle Informationsbroschüren und Selbsthilfematerialien ausgelegt werden. In diesem Zusammenhang soll kurz darauf hingewiesen werden, dass diese Materialen von den Pflegekräften auch gezielt eingesetzt werden können, wenn Beratungen durch die situativen Gegebenheiten des Stationsalltages oder die gegenwärtige Empfänglichkeit des Patienten für Entwöhnungsmaßnahmen gerade nicht möglich oder vermindert sind. Dem Einsatz solcher Broschüren konnte eine, wenn auch nur geringe, Wirksamkeit 
nachgewiesen werden (Lancaster und Stead 2005b), wobei ihre Verwendung die Pflegekräfte keinesfalls von der Pflicht entbinden sollte, persönliche Beratungen durchzuführen. Unter den Punkt der Umfeldbeeinflussung fallen weiterhin ein striktes Rauchverbot auf dem Stationsbalkon sowie die leichte Verfügbarkeit der Entwöhnungspräparate.

- Modelling: Durch das Vorführen von musterhaften, patientenzentrierten Beratungsgesprächen können Leitbilder und Beispiele dargeboten werden, an denen sich die Mitarbeiter bei zukünftigen Interventionen orientieren können. Diese exemplarischen Beratungen könnten auch im Rahmen von Videobeispielen vorgestellt, diskutiert und schließlich praktisch umgesetzt werden. Besonders überzeugende Rollenmodelle sind Klinikleiter und leitende Oberärzte, die öffentlich für das Thema Raucherentwöhnung eintreten und bei der Versorgung eigener Patienten die Leitlinie in Vorbildfunktion selbst umsetzen.

- Ermöglichung: Eine Ermöglichung des gewünschten Verhaltens kann in erster Linie durch Maßnahmen zur Umfeldbeeinflussung erreicht werden (s.o.). Das Ziel dieser Bemühungen sollte sein, den Pflegekräften diverse Möglichkeiten zu geben, das Thema Rauchen mit ihren Patienten zu besprechen. Ebenfalls sollte den Patienten der Zugang zu dieser Beratung erleichtert werden. Entsprechende Einzelmaßnahmen wurden bereits erörtert.

\subsubsection{Fertigkeiten}

In der Einleitung wurde bereits auf die unzureichende Vermittlung von Inhalten und Fertigkeiten auf dem Gebiet der Tabakabhängigkeit während der medizinischen Ausbildung hingewiesen (siehe Kapitel 1.7). Dies spiegelte sich auch in der Mitarbeiterbefragung in Phase I wieder, wonach sich lediglich rund $14 \%$ der Befragten ausreichend kompetent bei der Tabakentwöhnungsberatung einschätzten. Nahezu identische Werte lieferte eine aktuelle Befragung an einer anderen deutschen Universitätsklinik (Jahne et al. 2013).

Im Verlauf der hier vorgelegten Studie stellte sich heraus, dass eine einmalig durchgeführte Trainingsmaßnahme dies nicht wesentlich zu steigern vermochte und die entsprechenden Fertigkeiten und Inhalte der SOP auf diese Weise nicht nachhaltig vermittelt wurden. Zu berücksichtigen ist hierbei, dass die Teilnahmequote nicht besonders hoch war und zudem für die ärztlichen Mitarbeiter keine spezielle Schulung zur Vermittlung entsprechender Beratungskompetenzen stattfand. Eine verpflichtende Teilnahme daran wäre in diesem Fall in Erwägung zu ziehen, nachdem dies bisher auf fakultativer Basis stattfand. 
Abgeleitet aus dem Modell des $B C W$ können im Falle der kognitiven Fertigkeiten drei Interventionen (Training, Bildung und Ermöglichung) zur Beeinflussung und Optimierung dieser herangezogen werden.

- Training: Als naheliegendste Maßnahme käme ein erneutes Mitarbeitertraining in Betracht, um den meisten diesbezüglich identifizierten Defiziten begegnen zu können. Ein wesentlicher Schwerpunkt sollte dabei vor allem auf dem Einsatz von sowie dem Umgang mit den Nikotinersatzpräparaten liegen, da hier die größten Unsicherheiten und Schwierigkeiten berichtet wurden und sich ihre Anwendung weiterhin auf einem niedrigen Niveau befindet. Vergleichbare Schwierigkeiten und Kompetenzlücken konnten auch, ebenfalls im Rahmen einer qualitativen Erhebung mit Hilfe von Interviews und FokusgruppenDiskussionen, in anderen Krankenhäusern reproduziert werden (Schultz et al. 2011).

Weiterer Schwerpunkt zukünftiger Schulungen sollte die Förderung der Beratungskompetenz sein. Selbst wenn diese Studie keinen expliziten Hinweis auf schlechte Beratungen liefert oder einen entsprechenden Qualitätsindikator dafür, so muss es zur Sicherstellung einer nachhaltig hohen Beratungsqualität gerade unter Verweis auf andere Studien, bei denen oft speziell geschultes und teilweise langjährig ausgebildetes Personal eingesetzt wurde (Murray et al. 2013, Reid et al. 2006, Stevens et al. 1993), zwangsläufig zu weiteren Trainingsmaßnahmen kommen. Die von den Mitarbeitern geäußerte, mit Laufe des Projektes abnehmende Tendenz von Entwöhnungsaktivitäten geht in diese Richtung und impliziert dahingehenden Handlungsbedarf. Ein weiterer Hinweis, wenn auch nur auf hypothetischer Grundlage, könnte die bereits beschriebene ablehnende Haltung der Patienten auf die möglicherweise wenig empathischen bzw. insuffizienten Beratungen durch die Pflegekräfte sein. Ähnliche Patientenreaktionen beobachteten Butler und Kollegen als Folge einer mangelhaften ärztlichen Gesprächsführung (Butler et al. 1998).

Dabei ist es gerade die Beratung, die einen Zugang zu dem Raucher ermöglicht und eine Vertrauensbasis schafft, die Anstöße gibt, Motivation entwickelt und aufrecht erhält sowie Hilfe und Unterstützung bietet. Dies stellt zweifelsohne hohe Anforderungen an die kommunikativen Fähigkeiten des Beraters. Authentizität, Empathie, Fingerspitzengefühl und ein gewisses Repertoire an Beratungstechniken sind unverzichtbar. Eine amerikanische Studie konnte mit Hilfe von Fokusgruppen-Diskussionen unter Pflegekräften als eine der Hauptbarrieren bezüglich der Erteilung von Ratschlägen zum Rauchverzicht das Fehlen genau dieser konkreten Gesprächstechniken identifizieren (McCarty et al. 2001). In Anlehnung an die Arbeit von Cornuz und Mitarbeitern (siehe Kapitel 4.3) sollten deshalb vor allem praxisnahe und interaktive Elemente wie Rollenspiele, Videoanalysen und Fallbei- 
spiele zum Einsatz kommen, die den Mitarbeitern das verbale Rüstzeug und einen roten Faden für Entwöhnungsberatungen an die Hand geben.

Mehrere Schulungen, beispielsweise in halbjährlichen Intervallen, sorgen darüber hinaus für eine gleichbleibende Qualität und vermitteln die Relevanz und Bedeutung jener Entwöhnungsinterventionen bei hospitalisierten Rauchern. Eine Befragung pflegerischer Mitarbeiter in Arztpraxen zeigte, dass all diejenigen mehr Wissen und positivere Einstellungen in Bezug auf die Tabakentwöhnung hatten sowie deutlich häufiger Entwöhnungsaktivitäten unternahmen, die ein entsprechendes Training absolviert hatten (McEwen und West 2001). Ganz allgemein verbessert sich durch derartige Schulungsmaßnahmen medizinischer Kräfte nicht nur die Häufigkeit der Tabakentwöhnungsinterventionen, sondern auch deren Qualität (Carson et al. 2012, Cummings et al. 1989).

Gemäß der im Rahmen der vorliegenden Arbeit durchgeführten Mitarbeiterinterviews wurde eine nochmalige Schulung von den Mitarbeitern mehrheitlich befürwortet.

- Bildung: Mehrere befragte Mitarbeiter der UMG unterstellten den Entwöhnungsmedikamenten unter anderem eine fehlende Wirksamkeit, während die Bedeutung der Willenskraft bei einem Aufhörversuch in gleicher Weise überschätzt wurde. Diese verbreitete, fehlerhafte Einschätzung wurde bereits im Vorfeld durch andere Arbeiten bestätigt (Raupach et al. 2011, Raupach et al. 2013) und auch im Rahmen dieses Projektes als wesentliche Barriere im Hinblick auf den Einsatz einer medikamentösen Begleittherapie identifiziert.

Das Wissen um die Effektivität und die Bedeutung der unterstützenden Pharmakotherapie kann dabei dem Begriff der kognitiven oder intellektuellen Fertigkeiten des „COM- $B^{“ 6}$ Modells zugeordnet werden und gemäß dem $B C W$ mit entsprechenden Bildungsinterventionen beeinflusst werden. Letztere könnten in weitere Schulungen integriert oder im Rahmen von Kurzvorträgen während Mitarbeiterbesprechungen realisiert werden. Denn nur wenn die Mitarbeiter von der Effektivität der Präparate überzeugt sind, werden sie diese auch einsetzen. In einer neuseeländischen Klinik führten solche regelmäßig durchgeführten Bildungsmaßnahmen zu einem deutlichen Anstieg des Einsatzes der Nikotinersatzmittel (Vega und Stolare 2010).

- Ermöglichung: Voraussetzung für den Einsatz der Entwöhnungsmedikamente ist neben den oben genannten Aspekten auch eine ausreichende Bereitstellung der Präparate. Dies hat zumindest zu Beginn des Projektes nicht reibungslos funktioniert und möglicherweise zu der niedrigen Anzahl an Verordnungen geführt. Die Sicherstellung der jederzeitigen Verfügbarkeit muss zukünftig gewährleistet und ausreichend kommuniziert werden. 
Auf dem Gebiet der physischen Fertigkeiten konnten keine limitierenden Faktoren ermittelt werden, somit entfallen diesbezügliche Interventionsmaßnahmen. Zum Abschluss dieses Kapitels soll allerdings noch einmal darauf hingewiesen werden, dass ein Trainingsmangel nicht der hauptsächliche Grund für das Unterlassen der Raucherberatung war. Die wesentlichen Limitationen lagen mehrheitlich im Bereich der Motivation.

\subsubsection{Gelegenheit}

Die drei zur Beeinflussung des Aspektes der Gelegenheit vorgeschlagenen Interventionsfunktionen aus dem Konzept des $B C W$ (Umfeldbeeinflussung, Restriktion und Ermöglichung; siehe auch Tab. 9) lassen erkennen, dass es sich hier um überwiegend prozessuale und strukturelle Maßnahmen handelt, die die Voraussetzungen für eine nachhaltig integrierte Tabakentwöhnungsbehandlung hospitalisierter Raucher schaffen sollen. Für beide Unterformen der Gelegenheit (physisch und sozial) werden dabei dieselben Funktionen aufgeführt, wobei die Ergebnisse der Interviews nahezu ausschließlich Defizite im Bereich der sozialen Gelegenheit aufzeigten. Nachfolgend werden Vorschläge zur Beeinflussung der Gelegenheit dargestellt:

- Umfeldbeeinflussung: Darunter einzuordnen wäre zum einen die in den MitarbeiterInterviews geäußerte kritische Anmerkung, dass sowohl für die Patienten als auch die Mitarbeiter weiterhin die Möglichkeit bestünde, an der UMG bzw. direkt auf dem Stationsbalkon zu rauchen. Eine strikt rauchfreie Umgebung wurde in der Einleitung (siehe Kapitel 1.6) als bedeutender Faktor der sogenannten „günstigen Bedingungen“ herausgestellt, die wiederum als starkes Argument für die Initiierung einer Tabakentwöhnungsbehandlung im stationären Bereich angeführt wurden (Rigotti et al. 2007). Die Implementierung und allzeitige Sicherstellung eines Rauchverbotes insbesondere auf dem stationseigenen Balkon dient nicht nur der Herstellung dieser Bedingungen, sondern auch der Vermittlung des Bildes von Entschlossenheit und Kongruenz in Bezug auf die klinikeigenen Werte und das erklärte Ziel, Patienten bei der Überwindung ihrer Tabakabhängigkeit zu unterstützen. Zur Realisierung dessen und der Umsetzung entsprechender Standards trägt das Deutsche Netz Rauchfreier Krankenhäuser und Gesundheitseinrichtungen (DNRfK) in entscheidender Weise bei (Muhlig et al. 2013).

Weitere Maßnahmen, die die Beeinflussung des Umfeldes betreffen, sollten den schnellen und leichten Zugriff auf sämtliche SOP-Inhalte in Form von Aushängen gewährleisten. Entsprechende Defizite wurden von den Mitarbeitern vereinzelt kritisiert. Dazu gehört die 
Darstellung der diversen Aufgabenbereiche und Abläufe, Medikamenteninformationen einschließlich Dosierungshilfen sowie Kontaktnummern der Entwöhnungsambulanz bzw. von weiteren Ansprechpartnern. Da einige Mitarbeiter zwischen den verschiedenen Stationen rotieren, sollte sich der Aushang jeweils an derselben Stelle befinden, beispielsweise an der Tür des Stationszimmers. Ebenfalls sollte eine Aufnahme in die stationsüblichen Nachschlagequellen wie das Intranet erfolgen. Jeder Mitarbeiter muss darüber in Kenntnis gesetzt werden, wo die entsprechenden Informationen zu finden sind.

Als zusätzliche strukturelle Maßnahme muss die interstationäre Zusammenarbeit optimiert werden. Bei einem nicht unwesentlichen Anteil an Patienten erfolgt die initiale Behandlung auf der IMC-Station 1025, bevor diese im Anschluss zu Weiterbehandlungszwecken auf eine der beiden Normalstationen gelangen. In den Interviews wurde von ärztlichen Mitarbeitern der IMC-Station angemerkt, dass, bedingt durch die kurze Liegedauer von oftmals wenigen Tagen oder manchmal nur Stunden, eine kontinuierliche Betreuung erschwert würde und sie die Patienten „aus den Augen verlieren“ würden. Gerade für die weiterbehandelnde Station ist es aber relevant, ob und welche Entwöhnungsmaßnahmen bereits stattgefunden haben und welche noch initiiert werden müssen. Die Übermittlung dieser Informationen könnte einerseits mündlich im Zuge der Patientenübergabe geschehen. Noch vorteilhafter wäre eine schriftliche Dokumentation, beispielsweise durch einen entsprechenden Vermerk auf dem Aufnahmebogen oder auf einem dafür konzipierten Zusatzblatt, welches als fester Bestandteil neben Krankengeschichte und Befundberichten mit der Patientenakte übergeben wird.

Die dortigen Informationen sollten schließlich auch Eingang in den Entlassungsbrief des Patienten finden. Auf diese Weise kann der weiterbehandelnde Haus- oder Facharzt direkt an die initiierten Maßnahmen anknüpfen und die Tabakentwöhnungsbehandlung fortsetzen. Wie die Ergebnisse dieser Studie zeigten, erfolgte eine entsprechende Dokumentation der Entwöhnungsinterventionen in den Arztbriefen nur in einigen wenigen Fällen.

Ein weiterer Vorschlag auf der Ebene struktureller Maßnahmen betrifft den Einsatz eines Entwöhnungsbeauftragten. In personeller Hinsicht könnte diese Aufgabe von einer Pflegekraft, eventuell von der leitenden Stationspflegekraft erfüllt werden, die wiederrum ein fundierteres Training absolviert haben muss. Die beauftragte Person sollte in besonderem Maße mit sämtlichen Abläufen und Inhalten der implementierten SOP vertraut sein und als direkter Ansprechpartner auf Station dienen, den die Mitarbeiter bei Unsicherheiten, Fragen und Kritik jederzeit kontaktieren können. Daneben könnte auf diese Weise eine 
gewisse Kontrolle und Koordination der Entwöhnungsmaßnahmen erfolgen. Einzelne Pflegekräfte könnten im Zweifelsfall auf noch ausstehende Interventionen aufmerksam gemacht werden, ebenso sollte für eine stete Verfügbarkeit der notwendigen Ressourcen gesorgt werden. Eine weitere potenzielle Aufgabe sollte die Rücksprache bzw. den regelmäßigen Kontakt mit der klinikeigenen Entwöhnungsambulanz einschließen.

Bei der Thematisierung der Beratungskompetenz der Mitarbeiter (siehe Kapitel 4.5.2) wurde bereits darauf hingewiesen, dass in vielen anderen Studien im Rahmen der Implementierung von Tabakentwöhnungsmaßnahmen jeweils professionelle und speziell geschulte Kräfte eingesetzt wurden. Auch bei der ausführlicheren Vorstellung der Arbeit von Murray (siehe Kapitel 4.4) wurde angemerkt, dass sich auf diese Weise gewisse Barrieren wie eine mangelnde Beratungskompetenz oder eine geringe Priorisierung seitens des Stammpersonals von vornherein ausschließen ließen (Murray et al. 2013). Diese durchaus vorteilhafte und zweckmäßige Vorgehensweise ist unter Berücksichtigung finanzieller und personalpolitischer Überlegungen allerdings nicht immer uneingeschränkt möglich und durchsetzbar. Umso wichtiger ist es im Falle der UMG, durch eine vermehrte Einbindung der Mitarbeiter der klinikeigenen Entwöhnungsambulanz in die Prozesse auf den Stationen die gegeben personellen Strukturen effizienter zu nutzen. So könnten die dort beschäftigten, speziell ausgebildeten Kräfte regelmäßige Visiten auf den betreffenden Stationen durchführen, um Problemfälle zu besprechen und unentschlossene oder gar entwöhnungsunwillige Raucher mit ihrer Erfahrung doch noch für eine Tabakentwöhnung gewinnen.

- Restriktion: Eine Förderung des gewünschten Verhaltens in Richtung vermehrter Entwöhnungsinterventionen durch die Mitarbeiter lässt sich durch restriktive Maßnahmen weniger gut erreichen.

- Ermöglichung: Zum Abschluss dieser Darstellung soll auf die Notwendigkeit einer adäquaten Bereitstellung finanzieller Ressourcen hingewiesen werden. Sie ist die wesentliche Voraussetzung für eine erfolgreiche Implementierung vieler der vorgeschlagenen Interventionen gleichermaßen wie für die Aufrechterhaltung der bereits im Zuge dieser Studie eingesetzten Maßnahmen und Infrastruktur. Dies betrifft neben einer eventuellen Beschäftigung von zusätzlichem Personal beispielsweise auch die Gewährleistung der computergestützten Dokumentation oder die Bezahlung der eingesetzten Entwöhnungsmedikamente. In diesem Zusammenhang bedarf es einer ausreichenden und vor allem auch nachhaltigen finanziellen Unterstützung. 


\subsection{Ausblick}

Sämtliche oben genannten Maßnahmen zur Überwindung identifizierter Barrieren sowie der Behebung nach wie vor bestehender Versorgungsdefizite müssen im größeren Kontext struktureller Vorkehrungen betrachtet werden.

Mit Hilfe der vorgeschlagenen Interventionsfunktionen lassen sich eine oder mehrere Komponenten des Verhaltens und schließlich auch dieses selbst beeinflussen. Die letztendliche Umsetzung dieser Interventionen ist jedoch an weitere Parameter und Bedingungen geknüpft, die übergeordneter Natur sind und im Modell des $B C W$ (siehe Abb. 4) die dritte Ebene der sogenannten policy categories darstellen. Sie sind die grundsätzlichen Voraussetzungen für die in dieser Arbeit abgeleiteten Vorschläge.

Einige der zu den policy categories gehörenden Elemente sind bereits integraler Bestandteil des in Göttingen implementierten Systems. Dazu zählt das Konzipieren und Erlassen von Richtlinien (guidelines), die die genauen Prozessabläufe regeln und als Handlungsanweisungen fungieren. Die an der UMG im Rahmen dieses Projektes eingeführte SOP trägt diesem Grundsatz Rechnung. Von dem generellen Procedere der stationären Tabakentwöhnung, der grundsätzlichen Aufgabenverteilung bis hin zu den Empfehlungen bezüglich des Medikamenteneinsatzes werden die einzelnen Handlungen in leitlinienähnlicher Form definiert.

Unter den Aspekt der Umfeldgestaltung (environmental/social planning) als weiterer übergeordneten Maßnahme fällt in erster Linie die Schaffung einer rauchfreien Umgebung, auf deren Bedeutung bereits mehrfach hingewiesen wurde. Das Prinzip „rauchfreies Krankenhaus“ wird an der UMG seit zehn Jahren umgesetzt, wobei 2007 die Zertifizierung mit dem BronzeZertifikat des DNRfK erfolgte. Dieser Weg muss konsequent weiter beschritten werden, insbesondere vor dem Hintergrund der kritischen Äußerungen einzelner Mitarbeiter. Die weitere Kooperation und enge Zusammenarbeit mit dem DNRfK sind dabei unabdingbar.

Andere Punkte aus den policy categories hingegen kommen gegenwärtig weitaus weniger zur Geltung und lassen ein mitunter noch erhebliches Verbesserungspotenzial erkennen.

So der große Bereich der Regelungen (regulation), bei dem es vornehmlich um das Etablieren genereller Prinzipien und Standards geht. In diese Richtung können die Schritte der amerikanischen „Joint Commission“ (JCAHO, Joint Commission on Accreditation of Healthcare Organization) interpretiert werden. Diese Organisation evaluiert und zertifiziert Leistungserbringer im Gesundheitssystem zum Zwecke der Qualitätssicherung medizinischer Leistungen. 
Dabei wurden zuletzt auch Maßnahmen zur Behandlung der Tabakabhängigkeit in den Bewertungskatalog aufgenommen, beispielsweise die Häufigkeit unternommener Ausstiegsberatungen bei Rauchern oder der Einsatz von Medikamenten. Eine hohe Qualität der Versorgung und eine nachfolgende Akkreditierung haben darüber hinaus einen Einfluss auf die Vergütung der Leistungserbringer.

Unabhängig von dem daran gekoppelten finanziellen Aspekt (fiscal measures) soll das Augenmerk hier auf den Bemühungen liegen, die Behandlung der Tabakabhängigkeit über gewisse Regelungen zu einem Qualitätsindikator medizinischer Leistungen zu machen, der allgemein akzeptiert und integriert wird. Auch das Ottawa-Modell legte den Rauchstatus der Patienten nach dem Krankenhausaufenthalt als Qualitätsmerkmal fest (Reid et al. 2006). In dieser Hinsicht sind an der UMG noch weitere Anstrengungen erforderlich.

Der ausschlaggebende Faktor für den weiteren Erfolg des mittels dieses Projektes eingeschlagenen Weges ist die Förderung und Unterstützung des gesamten Vorhabens durch die Führungskräfte der UMG. Dies umfasst die Bereitstellung finanzieller Ressourcen ebenso wie die Schaffung entsprechender Vorgaben und Regelungen.

Fast noch bedeutender in diesem Zusammenhang ist die Kommunikation und Vermittlung (communication) des Projektes einschließlich aller dazugehörigen Maßnahmen. Für alle Mitarbeiter und Patienten erkennbar, sollten sowohl die Klinikleitung als auch die Chef- und Oberärzte der einzelnen Stationen konsequent hinter der Idee und letztendlich auch der Umsetzung stehen, was einerseits die der Tabakentwöhnung gebührende Bedeutung und Relevanz vermittelt, andererseits aber auch eine gewisse Dynamik zu entfalten vermag und so wie im Falle des Ottawa-Modells ein maßgeblicher Faktor für den Erfolg ist. Dem gesamten Vorhaben würde auf diese Weise der nötige Vorschub geleistet und eine entsprechende Nachhaltigkeit gesichert.

Künftige Studien müssten einerseits die Realisierung, Praktikabilität und schließlich auch den Effekt der in Kapitel 4.5 vorgeschlagenen Interventionsmaßnahmen auf das Verhalten, insbesondere auf die Motivation der medizinischen Mitarbeiter und letztlich auch auf die Versorgungssituation hospitalisierter Raucher an der UMG überprüfen.

Auf der anderen Seite sollte zusätzlich nachgewiesen werden, inwiefern sich die Implementierung ausgewählter policy categories auf das Verhalten der Mitarbeiter auswirkt und über die Modifikation welcher Komponenten des „COM-B“-Modells dieser Effekt eintritt. 


\section{Zusammenfassung}

Tabakrauchen ist ein wesentlicher Risikofaktor bei der Genese zahlreicher kardiovaskulärer, pneumologischer und maligner Erkrankungen. Aufgrund der großen Bedeutung der Beendigung des Tabakkonsums auch für die Sekundär- und Tertiärprävention empfehlen die Fachgesellschaften, insbesondere bei Patienten mit kardiovaskulären Erkrankungen, die Initiierung einer Entwöhnungsbehandlung bereits während des stationären Aufenthaltes.

In Anlehnung an internationale Leitlinien wurden deshalb im Rahmen einer prospektiven Interventionsstudie auf drei kardiologischen Stationen der UMG Maßnahmen zur Gewährleistung einer systematischen Versorgung hospitalisierter Raucher implementiert. Die getroffenen Maßnahmen umfassten die Einführung von Standard Operating Procedures (SOP) zum leitlinienkonformen Management rauchender Patienten sowie eine begleitende Mitarbeiterschulung. Die Effektivität der Intervention sollte hinsichtlich der Beratungsfrequenz und -qualität evaluiert werden. Neben einer quantitativen Auswertung erfolgte auch eine qualitative Analyse. Zu diesem Zweck wurden nach Implementierung der SOP Mitarbeiter interviewt. Der qualitative Studienteil diente der Identifikation eventueller Hindernisse bei der Umsetzung der implementierten Maßnahmen, welche anhand des „COM-B“- Modells und ihren Verhaltensdeterminanten Motivation, Fertigkeiten und Gelegenheit interpretiert wurden.

Insgesamt konnten 150 Probanden in die Studie eingeschlossen werden (je 75 Patienten vor und nach SOP-Implementierung). Die Resultate des Vorher-Nachher-Vergleichs zeigten eine signifikante Erhöhung der Interventionshäufigkeit bei den Rauchern seitens des ärztlichen $(6,7 \%$ vs. $38,7 \%$; p < 0,001), nicht jedoch seitens des pflegerischen Personals (1,3\% vs. $2,7 \% ; \mathrm{p}=0,560)$. Die Beratungshäufigkeit lag auch nach Einführung der SOP weiterhin unter $40 \%$, ebenso konnte der Einsatz einer Entwöhnungsmedikation oder die Anbindung an die klinikinterne Entwöhnungsambulanz durch die Intervention nicht wesentlich gesteigert werden.

Die Auswertung der Interviews zeigte, dass die hauptsächlichen Barrieren auf dem Weg zu einer adäquaten Raucherversorgung dem Bereich der Motivation zuzuordnen waren (z.B. selektive Umsetzung der SOP, mangelhafte Rollenkongruenz insbesondere beim Pflegepersonal). Hindernisse in Bezug auf die Fertigkeiten betrafen den Umgang mit den Entwöhnungspräparaten sowie eine mangelhafte Beratungskompetenz; bezüglich des Aspektes der Gelegenheit ging es vor allem um die inkonsequente Umsetzung des Rauchverbots im Klinikum. 
Aus den in dieser Studie gewonnenen Daten lassen sich unter Anwendung des Behaviour Change Wheels Empfehlungen für künftige Interventionen ableiten. Unter anderem erscheinen Anreize zur Durchführung vermehrter Entwöhnungsinterventionen sowie eine konsequente Überprüfung der Umsetzung der SOP sinnvoll. Zudem werden Rollenmodelle für die leitliniengerechte Betreuung rauchender Patienten benötigt.

In dieser Studie konnte die Effektivität der Implementierung von Standard Operating Procedures für die Beratung hospitalisierter Raucherinnen und Raucher in der Kardiologie und Pneumologie nachgewiesen werden. Die Wirksamkeit der Intervention war jedoch begrenzt. Um im Interesse der Patienten eine leitlinienkonforme Entwöhnungsberatung sicherzustellen, sind weitere Maßnahmen erforderlich. 


\section{Anhang}

1) Informationsblatt zur Fragebogen-Erhebung

2) Einverständniserklärung zur Fragebogen-Erhebung

3) Erhebungsbogen Patienten-Aufnahme

4) Erhebungsbogen Patienten-Entlassung

5) Erhebungsbogen Pflege vor Intervention

6) Erhebungsbogen Pflege nach Intervention

7) Informationsblatt zu den Interviews

8) Einverständniserklärung Interviews

9) Interview-Leitfaden für Ärztinnen/Ärzte

10) Interview-Leitfaden für Pflegekräfte

11) Aushang der SOP auf Station

12) Informationen bezüglich des Einsatzes der NRT auf Station 
Georg-August-Universität Göttingen • Bereich Humanmedizin Universitätsklinikum • Medizinische Fakultät

Zentrum Innere Medizin

Abteilung Kardiologie und Pneumologie

Direktor: Professor Dr. med. Gerd Hasenfuß

Studienleiter und Ansprechpartner:

Dr. med. T. Raupach

Abteilung Kardiologie \& Pneumologie

Universitätsmedizin Göttingen

Robert-Koch-Straße 40

37075 Göttingen

Telefon: 0551/398922

Mail: raupach@med.uni-goettingen.de

\section{Probanden/innen-Information zur Studie „Strukturiertes Training zur Tabakentwöhnung in einem Haus der Maximalversorgung: eine prospektive Studie“"}

Sehr geehrte Patientin, sehr geehrter Patient,

Sie werden heute zur Untersuchung und/oder Behandlung auf eine unserer Stationen aufgenommen - hierzu heißen wir Sie herzlich willkommen. Mit diesem Schreiben möchten wir Sie über eine wissenschaftliche Studie informieren, die zur Zeit auf unseren Stationen stattfindet. Zugleich möchten wir Sie herzlich dazu einladen, freiwillig an dieser Studie teilzunehmen und uns dabei zu helfen, die Versorgung unserer Patienten weiter zu verbessern. Sie können Ihre Teilnahme jederzeit ohne Angabe von Gründen beenden und Ihr Einverständnis mit der Datenspeicherung widerrufen.

In unserer Studie möchten wir untersuchen, wie sich eine Ausbildung der Ärztinnen und Ärzte sowie des Pflegepersonals zum Thema „Rauchen und Entwöhnung“ auf die Versorgung rauchender Patienten/innen auf unseren Stationen auswirkt. Dazu möchten wir Sie bitten, heute - am Aufnahmetag - einen kurzen Fragebogen auszufüllen. Am Vorabend Ihrer Entlassung werden wir Sie zu einem persönlichen Gespräch einladen, in dem wir Sie zu Ihrer Zufriedenheit mit dem Aufenthalt befragen. Das Gespräch dauert nur wenige Minuten.

Im Rahmen der Studie sollen einige persönliche Daten erhoben werden: Neben dem Geschlecht, dem Alter und der Aufnahmediagnose wird auch dokumentiert, ob Sie sich in unserer Entwöhnungsambulanz beraten lassen haben und ob eine entsprechende Dokumentation der Diagnose und des Therapie-Angebotes im Entlassungsbrief erfolgt ist. Diese Informationen werden allerdings nicht in Verbindung mit Ihrem Namen gespeichert. Statt dessen werden alle Daten „pseudonymisiert“. Unter einer Pseudonymisierung versteht man die Verschlüsselung von Daten ohne Namensnennung - nur mit einem Nummern-Code. Die Zuordnung der Daten zu einer Person ist nur möglich, wenn hierfür der Schlüssel eingesetzt wird, mit dem die Daten pseudonymisiert wurden. Die personenbezogenen Daten werden unter besonderen Schutzvorkehrungen getrennt von den pseudonymisierten Daten aufbewahrt. Eine Entschlüsselung ist nur durch die verantwortlichen Studienärzte möglich. Die Datenspeicherungszeit beträgt 10 Jahre. Eine Datenweitergabe an Dritte findet nicht statt. Bei Widerruf der Studienteilnahme können Sie die Löschung der personenbezogenen Daten verlangen. Eine Veröffentlichung findet nur mit anonymen Daten statt. Wir möchten Sie herzlich bitten, uns die Verwendung Ihrer Daten zu gestatten. Selbstverständlich ist dies Ihre freiwillige Entscheidung. 


\section{Einverständniserklärung für Teilnehmer der Studie „Strukturiertes Training zur Tabakentwöhnung in einem Haus der Maximalversorgung: eine prospektive Studie"}

Studienleiter und Ansprechpartner:

Dr. med. T. Raupach

Abteilung Kardiologie \& Pneumologie

Universitätsmedizin Göttingen

Robert-Koch-Straße 40

37075 Göttingen

Telefon: 0551/398922

Mail: raupach@med.uni-goettingen.de

Name des/der Patienten/in

Ich wurde von einem/r Mitarbeiter/in vollständig über Wesen, Bedeutung und Tragweite der Datenerhebung aufgeklärt. Ich hatte die Möglichkeit, Fragen zu stellen, habe falls erforderlich Antworten erhalten und diese verstanden.

Mir ist bekannt, daß meine persönlichen Daten in pseudononymisierter Form elektronisch gespeichert werden. (Pseudonymisierung bedeutet Verschlüsselung von Daten ohne Namensnennung nur mit Nummern codiert. Die Zuordnung der Daten zu einer Person ist nur möglich, wenn hierfür der Schlüssel eingesetzt wird, mit dem die Daten pseudonymisiert wurden.) Die personenbezogenen Daten werden unter besonderen Schutzvorkehrungen getrennt von den pseudonymisierten Daten aufbewahrt.

Eine Entschlüsselung ist nur durch die verantwortlichen Studienärzte möglich. Die Datenspeicherungszeit beträgt 10 Jahre. Eine Datenweitergabe an Dritte findet nicht statt. Bei Widerruf der Studienteilnahme kann ich die Löschung der personenbezogenen Daten verlangen. Eine Veröffentlichung findet nur mit anonymen Daten statt. Die Verwendung der Angaben über meine Gesundheit erfolgt nach gesetzlichen Bestimmungen und setzt vor der Teilnahme an der Datenerhebung folgende freiwillig abgegebene Einwilligungserklärung voraus, d.h. ohne die nachfolgende Einwilligung kann ich nicht an der Datenerhebung teilnehmen:

Ich erkläre mich damit einverstanden, daß im Rahmen dieser Datenerhebung gesammelte Daten, insbesondere Angaben über meine Gesundheit, in Papierform und elektronisch in der Abteilung Kardiologie \& Pneumologie aufgezeichnet werden. Die Speicherung und Auswertung zu wissenschaftlichen Zwecken erfolgt in pseudonymisierter Form.

Ich bin damit einverstanden, daß in der Entwöhnungsambulanz der Universitätsmedizin Göttingen eine Nachfrage erfolgt, ob ich an einer Beratung teilgenommen habe. Diesbezüglich entbinde ich meinen behandelnden Arzt von der Schweigepflicht.

Ich habe eine Kopie der Probanden/innen-Information und dieser Einwilligungserklärung erhalten. Hiermit erkläre ich meine freiwillige Teilnahme.

Ort und Datum

Unterschrift des/der Probanden/in

Ort und Datum

Unterschrift des aufklärenden Arztes 


\section{Erhebungsbogen Patienten-Aufnahme}

1. Geschlecht: $\square \mathrm{m} \quad \square \mathrm{w}$

2. Alter: Jahre

3. Seit wie vielen Jahren rauchen Sie? seit Jahren

4. Wie viel rauchen Sie im Durchschnitt pro Tag: Zigaretten / Tag Zigarren od. Zigarillos / Tag Pfeifen / Tag Wasserpfeife/Woche Elektrische Zigaretten /Tag

5. Wussten Sie, dass das Uniklinikum Göttingen ein rauchfreies Krankenhaus ist?
$\square \mathrm{Ja}$
$\square$ Nein

6. Haben Sie in der Vergangenheit bereits versucht, mit dem Rauchen aufzuhören

$\square$ NEIN (weiter bei Frage 6)

$\square$ JA - falls ja: Was war Ihr damals Anlaß, mit dem Rauchen aufzuhören?

7. Wie motiviert sind Sie jetzt, mit dem Rauchen aufzuhören?

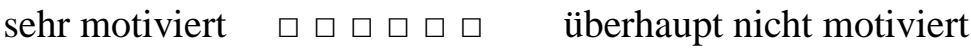

8. Wie leicht würde es Ihnen fallen, jetzt mit dem Rauchen aufzuhören?

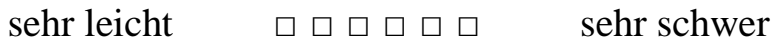

9. Wie sicher sind Sie, für mind. 4 Wochen mit dem Rauchen aufhören zu können?

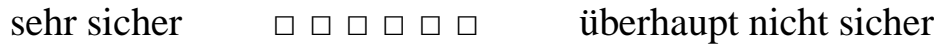

10. Sofern Sie mit dem Rauchen aufhören möchten: Wie hilfreich wären Ihrer Meinung nach unterstützende Maßnahmen (Beratung, Information, ggf. Medikamente, Vorstellung in der Tabakentwöhnungsambulanz....) im Rahmen Ihres jetzigen Klinikaufenthaltes?

$\square$ sehr hilfreich $\square$ hilfreich $\square$ wenig hilfreich

$\square$ gar nicht hilfreich, allein meine eigene Willenskraft ist entscheidend

11. Möchten Sie während Ihres Klinikaufenthaltes von den Ärztinnen/Ärzten bzw. vom Pflegepersonal zum Thema „Rauchentwöhnung“ beraten werden?
$\square \mathrm{Ja}$
$\square$ Nein
$\square$ Weiß nicht 


\section{Erhebungsbogen Patienten-Entlassung}

1. Wurden Sie während dieses Krankenhaus-Aufenthaltes hinsichtlich einer Tabakentwöhnung beraten?

$\square$ NEIN (weiter bei Frage 3)

$\square$ JA, von einer Schwester / einem Pfleger

$\square$ JA, von einer Ärztin / einem Arzt

Falls JA:

a) Ich fand die Beratung gut, weil

b) Ich fand die Beratung nicht gut, weil

2. Jetzt geht es um Ihre Zufriedenheit mit der Beratung durch Ärzte und Pflegekräfte:

a) Das Personal hat mich motiviert, mit Rauchen aufzuhören.

trifft voll zu trifft nicht zu

b) Ich fühlte mich zum Thema „Rauchen“ gut aufgeklärt und beraten

c) Ich fühlte mich durch Ratschläge zu meinem Rauchverhalten gestört/belästigt.

d) Ich hätte gern mehr Informationen zu den Möglichkeiten einer Tabakentwöhnung erhalten.

e) die Beratung zum Rauchstopp ist wichtig für meine Genesung

3. Wie motiviert sind Sie jetzt, mit dem Rauchen für immer aufzuhören?

sehr motiviert $\quad \square \quad \square \quad \square \square \quad$ überhaupt nicht motiviert

4. Haben Sie versucht, während Ihres Aufenthaltes mit dem Rauchen aufzuhören oder das Rauchen zu reduzieren?

$\square$ NEIN (weiter bei Frage 6)

$\square$ JA - falls ja: a) Was waren Ihre wichtigsten Gründe?

b) Was war dabei besonders hilfreich?

c) Welche Hilfsmittel haben Sie erhalten, um mit dem Rauchen aufzuhören? (mehrere Anworten möglich):

$\square$ keine

$\square$ Broschüre

$\square$ Telefonberatung (,Fax to quit")

$\square$ Nikotinersatzmittel (Pflaster, Kaugummi, Nasenspray...)

$\square$ einen Termin in der Entwöhnungsambulanz

$\square$ andere:

5. Wie sicher sind Sie, für mind. 4 Wochen mit dem Rauchen aufhören zu können? sehr sicher $\quad \square \quad \square \quad \square \square \quad$ überhaupt nicht sicher

6. Hatten Sie während Ihres stationären Aufenthaltes einen Termin in der Entwöhnungsambulanz des Klinikums?

$\square \mathrm{Ja} \quad \square$ Nein

7. Haben Sie vor, in den nächsten vier Wochen an einem Entwöhnungskurs in der Ambulanz des Klinikums teilzunehmen?

$\square$ Ja $\square$ Nein 
8. Das Klinikum ist ein „rauchfreies Krankenhaus“. Hatten Sie während Ihres Aufenthaltes den Eindruck, daß das konsequent umgesetzt wird?

$\square \mathrm{Ja} \quad \square$ Nein $\quad \square$ keine Meinung

Ihre Anmerkung dazu:

\section{Vom Studienpersonal auszufüllen:}

Aufenthaltsdauer: Tage

Hauptdiagnose laut Arztbrief:

Rauchen als Diagnose erwähnt:

$\square \mathrm{JA}$

$\square$ NEIN

Raucherstatus im Aufnahmebogen dokumentiert:

$\square$ JA $\quad \square$ NEIN

Entwöhnungsintervention in der Kurve dokumentiert:

$\square \mathrm{JA}$

$\square$ NEIN

Hilfsmittel zur Entwöhnung verordnet:

$\square \mathrm{JA}$

$\square$ NEIN

falls JA: welches?

$\square$ NET $\square$ Vareniclin $\square$ Bupropion

Ärztliche Anordnung zur Vereinbarung eines Ambulanztermins: $\quad \square$ JA $\quad \square$ NEIN 


\section{Erhebungsbogen Pflege vor Intervention}

1. Wie wichtig ist es für Sie zu wissen, ob ein/e Patient/in Raucher ist? sehr wichtig $\quad \square \quad \square \quad \square$ gar nicht wichtig

2. Hat es auf Ihre Stationsarbeit oder auf Ihren Umgang mit den Patienten/innen einen Einfluß, ob Patienten Raucher sind oder nicht?

$\square \mathrm{Ja}$, und zwar:

$\square$ Nein

3. Wie viele Ihrer rauchenden Patienten/innen beraten Sie bezüglich einer Tabakentwöhnung?
$\square 75-100 \%$
$\square 50-75 \%$
$\square 25-50 \%$
$\square 5-25 \%$
$\square$ weniger als $5 \%$

4. Wie kompetent fühlen Sie sich bei der Beratung rauchender Patienten? sehr kompetent $\square \square \square \square \square \square \quad$ gar nicht kompetent

5. Wissen und Fähigkeiten bezüglich der Beratung von Rauchern/innen trifft voll zu trifft nicht zu

a) Ich habe umfassendes Wissen über die Grundlagen der Tabakabhängigkeit.

b) Ich kenne die Folgeerkrankungen des Rauchens und anhaltenden Tabakkonsums.

c) Ich kenne verschiedene verhaltensbezogene und medikamentöse Maßnahmen zur Unterstützung der Tabakentwöhnung.

d) Ich kann Patienten/innen motivieren, mit dem Rauchen aufzuhören.

6. Bitte bewerten Sie die folgende Aussage:

stimme voll zu stimme nicht zu

Raucher/innen sollten bereits während ihres Klinikaufenthaltes zur Tabakentwöhnung beraten werden

7. Welchen Effekt hat es Ihrer Meinung nach für die Genesung und Förderung der Gesundheit Ihrer Patienten/innen, wenn Sie Raucher/innen während ihres Aufenthaltes zum Thema „Rauchfrei werden / Tabakentwöhnung“ beraten?

einen großen Effekt $\quad$ $\quad \square \quad \square \quad$ gar keinen Effekt

8. Wie groß ist der zeitliche Aufwand durch die Beratung rauchender Patienten/innen für Sie?

Pro Beratung... $\quad \square$ 0-5 min $\quad \square 5-10 \mathrm{~min} \quad \square 10-20 \mathrm{~min} \quad \square>20 \mathrm{~min}$

9. Sind Sie selbst Raucher?

$\square \mathrm{Ja}$

$\square$ Nein - ich habe noch nie geraucht

$\square$ Nein - ich habe früher geraucht aber mittlerweile aufgehört 


\section{Erhebungsbogen Pflege nach Intervention}

1. Haben Sie an dem Trainingsprogramm zur Raucherberatung teilgenommen?

$\square$ NEIN (weiter bei Frage 2)

$\square \mathrm{JA}$ - falls ja:

a) Was hat Ihnen daran gut gefallen?

b) Was hat Ihnen daran nicht gefallen?

c) Bitte geben Sie dem Training eine Note ( $1=$ sehr gut, $6=$ ungenügend):

d) Bitte bewerten Sie die folgende Aussage:

„Um meine Kenntnisse und Fähigkeiten bezüglich der Raucherberatung zu festigen, wäre eine erneute Schulung in einem gewissen Abstand sinnvoll.“

trifft voll zu $\quad \square \quad \square \quad \square \quad$ trifft nicht zu

2. Wie viele Ihrer rauchenden Patienten/innen beraten Sie bezüglich einer Tabakentwöhnung?
$\square 75-100 \%$
口 50-75\%
$\square 25-50 \%$
$\square-25 \%$
$\square$ weniger als $5 \%$

3. Wie kompetent fühlen Sie sich bei der Beratung rauchender Patienten/innen? sehr kompetent $\quad \square \quad \square \quad \square \quad$ gar nicht kompetent

4. Wissen und Fähigkeiten bezüglich der Beratung von Rauchern/innen

trifft voll zu trifft nicht zu

a) Ich habe umfassendes Wissen über die Grundlagen der Tabakabhängigkeit.

b) Ich kenne die Folgeerkrankungen des Rauchens und anhaltenden Tabakkonsums.

c) Ich kenne verschiedene verhaltensbezogene und medikamentöse Maßnahmen zur Unterstützung der Tabakentwöhnung.

d) Ich kann Patienten/innen motivieren, mit dem Rauchen aufzuhören.

5. Welchen Effekt hat es Ihrer Meinung nach für die Genesung und Förderung der Gesundheit Ihrer Patienten/innen, wenn Sie Raucher/innen während ihres Aufenthaltes zum Thema „Rauchfrei werden / Tabakentwöhnung“ beraten?

einen großen Effekt $\quad$ $\quad \square \quad \square \quad$ gar keinen Effekt

6. Welche der folgenden Reaktionen haben Sie von Patienten/innen erhalten, die Sie zum Thema „Rauchfrei werden / Tabakentwöhnung“ beraten haben?

Patient/in zeigt Interesse

Patient/in wünscht mehr Information/Beratuung

Patient/in ist zum Rauchstopp bereit

Patient/in hört mit dem Rauchen auf

Patient/in lehnt die Beratung ab

Sonstige: häufig $\square \square \square \square \square \square$ nie

häufig $\square \square \square \square \square \square$ nie

häufig $\square \square \square \square \square \square$ nie

häufig $\square \square \square \square \square \square$ nie

häufig $\square \square \square \square \square \square$ nie 
7. Wie groß ist der zeitliche Aufwand durch die Beratung rauchender Patienten/innen für Sie?

Pro Beratung... $\square$ 0-5 min $\square 5-10 \mathrm{~min} \quad \square 10-20 \mathrm{~min} \quad \square>20 \mathrm{~min}$

8. Bitte beurteilen Sie die folgenden Aussagen zu dieser zusätzlich anfallenden Arbeit auf Station, seit es die „SOPs“ für die Betreuung von Rauchern/innen gibt:

a) Diese zusätzliche Arbeit ließ sich gut in den trifft voll zu trifft nicht zu normalen Arbeitsalltag integrieren.

b) Mit der Zeit wurde ich routinierter und konnte die zusätzliche Arbeit gut in den Alltag integrieren.

c) Ich kann mir gut vorstellen, auch weiterhin rauchende Patienten/innen auf der Station zu betreuen.

d) Man sollte diese Maßnahmen auch auf den anderen Stationen einführen.

e) Ich kann mir gut vorstellen, meine Kollegen/innen zur Beratung zu motivieren.

9. Bemerkungen, Anregungen, Sonstiges:

10. Sind Sie selbst Raucher?

$\square \mathrm{Ja}$

$\square$ Nein - ich habe noch nie geraucht

$\square$ Nein - ich habe früher geraucht aber mittlerweile aufgehört 
Georg-August-Universität Göttingen • Bereich Humanmedizin

Universitätsklinikum • Medizinische Fakultät

Zentrum Innere Medizin

Abteilung Kardiologie und Pneumologie

Direktor: Professor Dr. med. Gerd Hasenfuß

Studienleiter und Ansprechpartner:

PD Dr. med. T. Raupach, MME Ab-

teilung Kardiologie \& Pneumologie

Universitätsmedizin Göttingen Ro-

bert-Koch-Straße 4037075 Göttin-

gen Telefon: 0551/398922 Mail:

raupach@med.uni-goettingen.de

\section{Probanden/innen-Information zur Studie „Strukturiertes Training zur Tabakentwöhnung in einem Haus der Maximalversorgung: eine prospektive Studie - Interviews zur Evaluation"}

Sehr geehrte/r Gesundheits- und Krankenpfleger/in, sehr geehrte/r Arzt/Ärztin,

Sie waren zwischen Februar 2011 und August 2011 auf den Stationen der Abteilung Kardiologie \& Pneumologie $(1025,2021,2022)$ tätig. Im Mai 2011 wurde eine neue „Standard Operating Procedure (SOP)“ zur Betreuung rauchender Patienten/innen eingeführt, und Sie hatten die Möglichkeit, an einer entsprechenden Schulungsmaßnahme teilzunehmen. In einem Anschlußprojekt soll die SOP nun evaluiert (bewertet) werden. Dazu möchten wir Sie in einem ca. 30minütigen Interview nach Ihren Erfahrungen mit der Schulung und der Umsetzung der SOP fragen. Aus den hierbei erhobenen Informationen wollen wir Ansätze zur Verbesserung der SOP ableiten. Die Interviews dienen zusätzlich der Erforschung möglicher Probleme bei der Umsetzung von SOPs zur Betreuung rauchender Patienten.

Für das Interview wird ein Leitfaden verwendet, der aus neun Hauptfragen besteht. An diese schließen sich - je nach Bedarf - Unterfragen an. Das Interview wird von einer Psychologin geführt, die selbst nicht an der Entstehung der SOP beteiligt war und die in keinem Bezug zur Pflegedienstleitung oder zum ärztlichen Abteilungsleiter steht. Das Interview wird akustisch-elektronisch aufgezeichnet und von einer dritten Person wörtlich abgeschrieben. Diese Person hat keinen Bezug zur Patientenversorgung, zur SOP, zur Pflegedienstleistung oder zur ärztlichen Abteilungsleitung. Bei der Abschrift des Interviews werden Ihr Geschlecht und Ihre Zugehörigkeit zum Pflegedienst bzw. ärztlichen Dienst registriert; weitere persönliche Daten werden nicht registriert.

Die zur Auswertung verwendete Abschrift des Interviews wird keine Hinweise auf Ihre Person enthalten. Die akustisch-elektronische Aufzeichnung wird nach der Abschrift gelöscht.

Ihre Teilnahme am Interview ist freiwillig; alle Äußerungen geschehen auf rein freiwilliger Basis. Sie können das Interview jederzeit beenden und verlangen, daß alle bis zu diesem Zeitpunkt gemachten Aufnahmen direkt gelöscht werden, ohne abgeschrieben zu werden. Ihre Aussagen werden nicht an die Pflegedienstleitung oder den ärztlichen Abteilungsdirektor weitergegeben.

PD Dr. med. T. Raupach, MME (Studienleiter) 


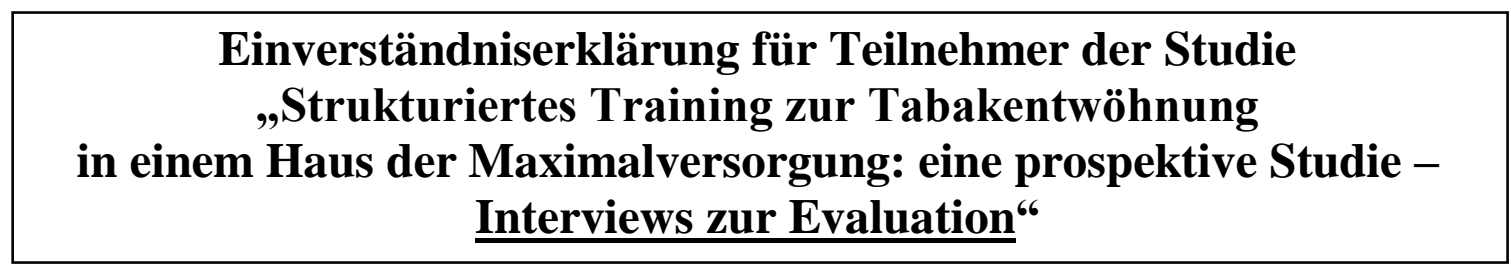

Studienleiter und Ansprechpartner:

PD Dr. med. T. Raupach, MME

Name des/der Studienteilnehmers/in

Abteilung Kardiologie \& Pneumologie

Universitätsmedizin Göttingen

Robert-Koch-Straße 40

37075 Göttingen

Telefon: 0551/398922

Mail: raupach@med.uni-goettingen.de

Ich wurde von einem/r Mitarbeiter/in vollständig über Wesen, Bedeutung und Tragweite der Datenerhebung aufgeklärt. Ich hatte die Möglichkeit, Fragen zu stellen, habe falls erforderlich Antworten erhalten und diese verstanden.

Mir ist bekannt, daß im Rahmen des Interviews keine persönlichen Daten von mir erhoben oder gespeichert werden. Das Interview wird jedoch akustischelektronisch aufgezeichnet und danach von einer dritten Person transkribiert (d.h. wörtlich abgeschrieben). Diese Person steht in keinem Zusammenhang mit dem strukturierten Training zur Tabakentwöhnung. Sie wird das Interview transkribieren, ohne zu wissen, von wem es gegeben wurde. Die Abschrift des Interviews wird lediglich eine Information zu meinem Geschlecht und zu meiner Berufsgruppe enthalten; andere persönliche Daten werden nicht erhoben. Die Auswertung des transkribierten Interviews erfolgt somit ohne jeglichen Bezug zu meiner Person. Das elektronisch gespeicherte Interview wird nach der Transkription gelöscht.

Ich erkläre mich damit einverstanden, daß das mit mir geführte Interview akustisch-elektronisch gespeichert wird. Nach Abschrift des Interviews wird die Datei umgehend gelöscht.

Ich habe eine Kopie der Probanden/innen-Information und dieser Einwilligungserklärung erhalten. Hiermit erkläre ich meine freiwillige Teilnahme.

Ort und Datum

Unterschrift des/der Probanden/in

Ort und Datum

Unterschrift des Studienleiters 


\section{Interview-Leitfaden für Ärztinnen/Ärzte}

Vorbemerkung: „In diesem Interview geht es um die SOP zur Raucherberatung, die im Mai 2011 auf den kardiologischen Stationen eingeführt wurde. Wir möchten Sie nach Ihren Erfahrungen mit der SOP befragen. Das Interview soll dazu dienen, die SOP mit Ihrer Hilfe zu verbessern. Ich selbst bin nicht in der Raucherentwöhnung aktiv und habe auch mit der SOP nichts zu tun. Das Interview wird aufgezeichnet und von einer dritten Person abgeschrieben, die ebenfalls keinen Bezug zur SOP hat. Erst dann erfolgt die Auswertung der Daten. Damit wird sichergestellt, daß alle Ihre Aussagen anonym bleiben und daß aus den Daten keine Rückschlüsse auf Ihre Person gezogen werden können.“

1) Was halten Sie von der SOP zur Betreuung rauchender Patienten auf der Station?

- Wie hoch schätzen Sie die Notwendigkeit zur Einrichtung dieser SOP ein?

- Wie nützlich ist die SOP Ihrer Meinung nach?

2) Nach der SOP sollen die Ärztinnen und Ärzte Kurzberatungen bei allen rauchenden Pati enten durchführen, gegebenenfalls Entwöhnungsmedikamente verschreiben und die Pati enten an die Entwöhnungsambulanz überweisen. Außerdem soll die Nomenklatur in den Arztbriefen vereinheitlicht werden, und die bezüglich des Rauchens eingeleiteten Maß nahmen sollen im Arztbrief dokumentiert werden.

- Wie denken Sie darüber?

- Wessen Aufgabe ist es Ihrer Meinung nach, mit Patienten über das Rauchen und die Entwöhnung zu sprechen?

- Was sehen Sie als Ärztin/Arzt als Ihre Rolle bei der Raucherberatung an?

- Gibt es Aspekte an der SOP, die Ihnen nicht zusagen?

- Haben Sie Vorschläge, wie die SOP verbessert werden könnte?

3) Haben Sie seit der Einführung der SOP irgendwelche Veränderungen festgestellt?

- Was hat sich geändert?

4) Welche Auswirkungen hat die SOP auf Ihren Arbeitsalltag gehabt?

- Wie haben Sie die SOP umgesetzt? (falls das noch nicht beantwortet wurde)

5) Welche Erfahrungen haben Sie damit gemacht, das Thema „Rauchen“ bei Patienten anzu sprechen?

- Wie hat es sich angefühlt?

6) Was denken Sie über Medikamente zur Tabakentwöhnung?

- Wie kompetent fühlen Sie sich, den Patienten die Anwendung dieser Medikamente zu erklären?

7) Was denken Sie über die Informationen, die Sie zur Einführung der SOP per Mail erhalten haben?

- Wie gut sind Sie in der Lage, das Thema „Rauchen“ mit Patienten zu diskutieren?

- Gab es irgendwelche Schwierigkeiten bei der Umsetzung der Leitlinie?

- Wenn ja: Welche?

8) Glauben Sie, daß eine weitere Fortbildung zum Thema hilfreich wäre?

- Welche Aspekte sollten in einer solchen Fortbildung angesprochen werden?

9) Welchen Stellenwert hat Ihrer Meinung nach die Behandlung von Rauchern (Beratung und medikamentöse Therapie) in der UMG? 


\section{Interview-Leitfaden für Pflegekräfte}

Vorbemerkung: „In diesem Interview geht es um die SOP zur Raucherberatung, die im Mai 2011 auf den kardiologischen Stationen eingeführt wurde. Wir möchten Sie nach Ihren Erfahrungen mit der SOP befragen. Das Interview soll dazu dienen, die SOP mit Ihrer Hilfe zu verbessern. Ich selbst bin nicht in der Raucherentwöhnung aktiv und habe auch mit der SOP nichts zu tun. Das Interview wird aufgezeichnet und von einer dritten Person abgeschrieben, die ebenfalls keinen Bezug zur SOP hat. Erst dann erfolgt die Auswertung der Daten. Damit wird sichergestellt, daß alle Ihre Aussagen anonym bleiben und daß aus den Daten keine Rückschlüsse auf Ihre Person gezogen werden können.“

1) Was halten Sie von der SOP zur Betreuung rauchender Patienten auf der Station?

- Wie hoch schätzen Sie die Notwendigkeit zur Einrichtung dieser SOP ein?

- Wie nützlich ist die SOP Ihrer Meinung nach?

2) Nach der SOP sollen die Pflegekräfte den Raucherstatus der Patienten dokumentieren, rauchende Patienten an der Stationstafel kennzeichnen, die Handhabung der Medikamente erklären und gegebenenfalls einen Termin in der Entwöhnungsambulanz anmelden.

- Wie denken Sie darüber?

- Wessen Aufgabe ist es Ihrer Meinung nach, mit Patienten über das Rauchen und die Entwöhnung zu sprechen?

- Was sehen Sie als Pflegekraft als Ihre Rolle bei der Raucherberatung an?

- Gibt es Aspekte an der SOP, die Ihnen nicht zusagen?

- Haben Sie Vorschläge, wie die SOP verbessert werden könnte?

3) Haben Sie seit der Einführung der SOP irgendwelche Veränderungen festgestellt?

- Was hat sich geändert?

4) Welche Auswirkungen hat die SOP auf Ihren Arbeitsalltag gehabt?

- Wie haben Sie die SOP umgesetzt? (falls das noch nicht beantwortet wurde)

5) Welche Erfahrungen haben Sie damit gemacht, das Thema „Rauchen“ bei Patienten anzu sprechen?

- Wie hat es sich angefühlt?

6) Was denken Sie über Medikamente zur Tabakentwöhnung?

- Wie kompetent fühlen Sie sich, den Patienten die Anwendung dieser Medikamente zu er klären?

7) Was denken Sie über die Schulung, die Sie zur Einführung der SOP erhalten haben? (Falls die Fortbildung nicht besucht wurde: Woran lag das? Hätten Sie gerne mitgemacht?)

- Wie gut sind Sie in der Lage, das Thema „Rauchen“ mit Patienten zu diskutieren?

- Gab es irgendwelche Schwierigkeiten bei der Umsetzung der Leitlinie?

- Wenn ja: Welche?

8) Glauben Sie, daß eine weitere Fortbildung zum Thema hilfreich wäre?

- Welche Aspekte sollten in einer solchen Fortbildung angesprochen werden?

9) Welchen Stellenwert hat Ihrer Meinung nach die Behandlung von Rauchern (Beratung und medikamentöse Therapie) in der UMG? 


\section{SOP Raucherberatung}

Stationen: 2021, 2022 und 1025

\begin{tabular}{|c|c|c|}
\hline Zeitpunkt & Aktion & Verantwortung \\
\hline \multirow{3}{*}{ Aufnahme } & $\begin{array}{l}\text { Dokumentation des Rauchersta- } \\
\text { tus in der Planette }\end{array}$ & Pflege \\
\hline & Ziehen des grauen Reiters & Pflege \\
\hline & $\begin{array}{l}\text { Markierung des Patienten an der } \\
\text { Stationstafel } \\
\end{array}$ & Pflege \\
\hline \multirow{5}{*}{$\begin{array}{l}\text { Innerhalb von } 3 \\
\text { Tagen nach Auf- } \\
\text { nahme }\end{array}$} & Kurzberatung & Arzt/Ärztin \\
\hline & $\begin{array}{l}\text { Verordnung einer medikamentö- } \\
\text { sen Unterstützung }\end{array}$ & Arzt/Ärztin \\
\hline & $\begin{array}{l}\text { Erklärung der Handhabung der } \\
\text { Medikamente }\end{array}$ & Pflege \\
\hline & $\begin{array}{c}\text { Überweisung an die Entwöh- } \\
\text { nungsambulanz }\end{array}$ & Arzt/Ärztin \\
\hline & $\begin{array}{c}\text { Kontaktaufnahme mit der Ent- } \\
\text { wöhnungsambulanz }\end{array}$ & Pflege \\
\hline \multirow{2}{*}{ Entlassung } & $\begin{array}{c}\text { fachlich korrekte Dokumentation } \\
\text { des Rauchstatus im Arztbrief }\end{array}$ & Arzt/Ärztin \\
\hline & $\begin{array}{c}\text { Dokumentation eingeleiteter } \\
\text { Maßnahmen im Arztbrief }\end{array}$ & Arzt/Ärztin \\
\hline
\end{tabular}




\section{Empfehlungen zur Pharmakotherapie}

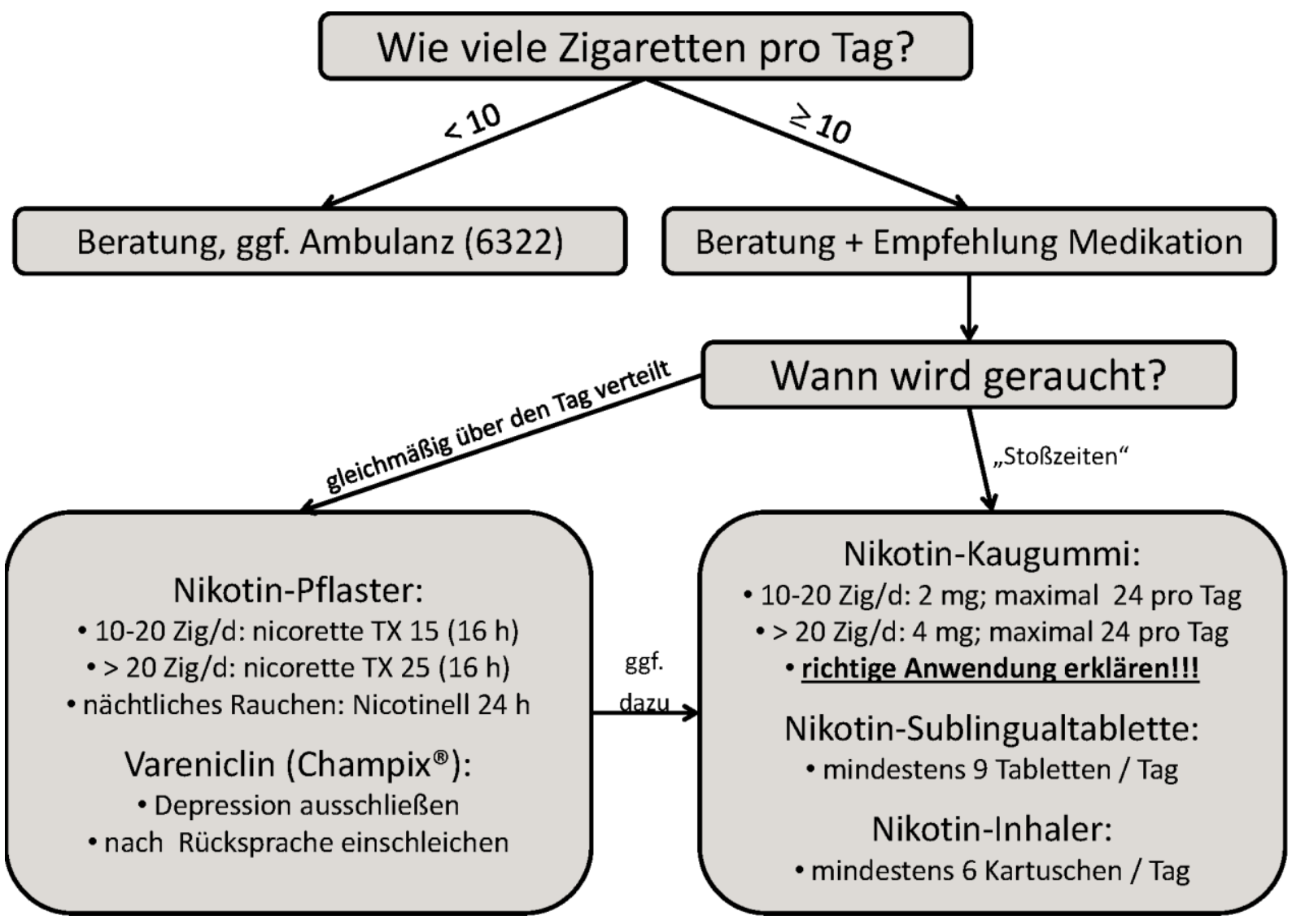

\section{Informationen zur Nikotinersatztherapie}

- Das Nicorette-Pflaster kann durchgeschnitten werden, das Nicotinell-Pflaster nicht.

- Mehrere Formen der Nikotinersatztherapie können miteinander kombiniert werden.

- empfohlene Mindest-Behandlungsdauer: 5 Wochen

- Ausschleichen ist möglich - aber nicht notwendig.

- Bei der Anwendung von Nikotinersatz bleibt der Nikotinspiegel unter demjenigen eines durchschnittlichen Aktivrauchers. 


\section{Literaturverzeichnis}

1. Althuis MD, Sexton M, Prybylski D (1999): Cigarette smoking and asthma symptom severity among adult asthmatics. J Asthma $\underline{36}$, 257-264

2. Anders S, Strobel L, Krampe H, Raupach T (2013): Do final-year medical students know enough about the treatment of alcohol use disorders and smoking? Dtsch Med Wochenschr 138, 23-27

3. Anderson JE, Jorenby DE, Scott WJ, Fiore MC (2002): Treating tobacco use and dependence: an evidence-based clinical practice guideline for tobacco cessation. Chest $\underline{121}, 932-941$

4. Andreas S, Batra A, Behr J, Berck H, Chenot JF, Gillissen A, Hering T, Herth F, Meierjurgen R, Muhlig S et al. (2008): Guidelines for smoking cessation in patients with COPD issued by the Deutsche Gesellschaft fur Pneumologie und Beatmungsmedizin. Pneumologie $\underline{62}$, 255-272

5. Anthonisen NR, Connett JE, Murray RP (2002): Smoking and lung function of Lung Health Study participants after 11 years. Am J Respir Crit Care Med 166, 675-679

6. Anthonisen NR, Skeans MA, Wise RA, Manfreda J, Kanner RE, Connett JE (2005): The effects of a smoking cessation intervention on 14.5-year mortality: a randomized clinical trial. Ann Intern Med 142, 233-239

7. Ascher JA, Cole JO, Colin JN, Feighner JP, Ferris RM, Fibiger HC, Golden RN, Martin P, Potter WZ, Richelson E et al. (1995): Bupropion: a review of its mechanism of antidepressant activity. J Clin Psychiatry $\underline{56}$, 395-401

8. Aubin HJ, Bobak A, Britton JR, Oncken C, Billing CB, Jr., Gong J, Williams KE, Reeves KR (2008): Varenicline versus transdermal nicotine patch for smoking cessation: results from a randomised open-label trial. Thorax $\underline{63}, 717-724$

9. Baillie AJ, Mattick RP, Hall W (1995): Quitting smoking: estimation by meta-analysis of the rate of unaided smoking cessation. Aust J Public Health $\underline{19}$, 129-131

10. Balfour DJ (1994): Neural mechanisms underlying nicotine dependence. Addiction $\underline{89}$, $1419-1423$

11. Balfour DJ, Fagerstrom KO (1996): Pharmacology of nicotine and its therapeutic use in smoking cessation and neurodegenerative disorders. Pharmacol Ther $\underline{72}, 51-81$

12. Ball K, Turner R (1974): Smoking and the heart. The basis for action. Lancet $\underline{2}$, 822826

13. Bartels C, Abuhaliga AR, McGee H, Morgan K, McElvaney NG, Doyle F (2012): A survey of the prevalence of smoking and smoking cessation advice received by inpatients in a large teaching hospital in Ireland. Ir J Med Sci 181, 445-449 
14. Barter CE, Campbell AH (1976): Relationship of constitutional factors and cigarette smoking to decrease in 1-second forced expiratory volume. Am Rev Respir Dis $\underline{113}$, 305-314

15. Barua RS, Ambrose JA, Eales-Reynolds LJ, DeVoe MC, Zervas JG, Saha DC (2001): Dysfunctional endothelial nitric oxide biosynthesis in healthy smokers with impaired endothelium-dependent vasodilatation. Circulation $\underline{104}$, 1905-1910

16. Batra A (2001): Bupropion - wirkungsvolle oder nebenwirkungsreiche Raucherentwöhnungstherapie? Sucht $\underline{47}$, 294-298

17. Batra V, Patkar AA, Berrettini WH, Weinstein SP, Leone FT (2003): The genetic determinants of smoking. Chest $\underline{123}, 1730-1739$

18. Benowitz NL (2010): Nicotine addiction. N Engl J Med 362, 2295-2303

19. Benowitz NL, Gourlay SG (1997): Cardiovascular toxicity of nicotine: implications for nicotine replacement therapy. J Am Coll Cardiol 29, 1422-1431

20. Benowitz NL, Jacob P, Jones RT, Rosenberg J (1982): Interindividual variability in the metabolism and cardiovascular effects of nicotine in man. J Pharmacol Exp Ther $\underline{221}, 368-372$

21. Benowitz NL, Porchet H, Sheiner L, Jacob P, 3rd (1988): Nicotine absorption and cardiovascular effects with smokeless tobacco use: comparison with cigarettes and nicotine gum. Clin Pharmacol Ther $\underline{44}$, 23-28

22. Benwell ME, Balfour DJ, Anderson JM (1988): Evidence that tobacco smoking increases the density of (-)-[3H]nicotine binding sites in human brain. J Neurochem $\underline{50}$, $1243-1247$

23. Bhang SY, Choi SW, Ahn JH, Kim K, Kim H, Park HK (2013): Predictors of success at six-month follow-up at a public smoking cessation clinic in South Korea. Asia Pac Psychiatry $\underline{5}, 197-204$

24. Bjartveit K, Tverdal A (2005): Health consequences of smoking 1-4 cigarettes per day. Tob Control 14, 315-320

25. Blann AD, Kirkpatrick U, Devine C, Naser S, McCollum CN (1998): The influence of acute smoking on leucocytes, platelets and the endothelium. Atherosclerosis $\underline{141}, 133-$ 139

26. Bolliger CT, Issa JS, Posadas-Valay R, Safwat T, Abreu P, Correia EA, Park PW, Chopra P (2011): Effects of varenicline in adult smokers: a multinational, 24-week, randomized, double-blind, placebo-controlled study. Clin Ther $\underline{33}, 465-477$

27. Bray FI, Weiderpass E (2009): Lung cancer mortality trends in 36 European countries: secular trends and birth cohort patterns by sex and region 1970-2007. Int J Cancer $\underline{126}, 1454-1466$ 
28. Brischetto CS, Connor WE, Connor SL, Matarazzo JD (1983): Plasma lipid and lipoprotein profiles of cigarette smokers from randomly selected families: enhancement of hyperlipidemia and depression of high-density lipoprotein. Am J Cardiol 52, 675-680

29. Brotons C, Bulc M, Sammut MR, Sheehan M, Manuel da Silva Martins C, Bjorkelund C, Drenthen AJ, Duhot D, Gorpelioglui S, Jurgova E et al. (2011): Attitudes toward preventive services and lifestyle: the views of primary care patients in Europe. The EUROPREVIEW patient study. Fam Pract 29 Suppl 1, i168-i176

30. Brunner H, Cockcroft JR, Deanfield J, Donald A, Ferrannini E, Halcox J, Kiowski W, Luscher TF, Mancia G, Natali A et al. (2005): Endothelial function and dysfunction. Part II: Association with cardiovascular risk factors and diseases. A statement by the Working Group on Endothelins and Endothelial Factors of the European Society of Hypertension. J Hypertens $\underline{23}$, 233-246

31. Buisson B, Bertrand D (2001): Chronic exposure to nicotine upregulates the human (alpha)4((beta)2 nicotinic acetylcholine receptor function. J Neurosci 21, 1819-1829

32. Burnett AL, Sharlip ID (2013): Standard operating procedures for priapism. J Sex Med $\underline{10}, 180-194$

33. Butler CC, Pill R, Stott NC (1998): Qualitative study of patients' perceptions of doctors' advice to quit smoking: implications for opportunistic health promotion. Bmj $\underline{316}, 1878-1881$

34. Cahill K, Stead LF, Lancaster T (2011): Nicotine receptor partial agonists for smoking cessation. Cochrane Database Syst Rev 2, CD006103

35. Carson KV, Verbiest ME, Crone MR, Brinn MP, Esterman AJ, Assendelft WJ, Smith BJ (2012): Training health professionals in smoking cessation. Cochrane Database Syst Rev $\underline{5}$, CD000214

36. Celermajer DS, Adams MR, Clarkson P, Robinson J, McCredie R, Donald A, Deanfield JE (1996): Passive smoking and impaired endothelium-dependent arterial dilatation in healthy young adults. N Engl J Med $\underline{334}$, 150-154

37. Chapman KR (2004): Chronic obstructive pulmonary disease: are women more susceptible than men? Clin Chest Med 25, 331-341

38. Chapman S (1993): The role of doctors in promoting smoking cessation. Bmj $\underline{307}$, 518-519

39. Chouinard MC, Robichaud-Ekstrand S (2005): The effectiveness of a nursing inpatient smoking cessation program in individuals with cardiovascular disease. Nurs Res $\underline{54}$, $243-254$

40. Clark MA, Hogan JW, Kviz FJ, Prohaska TR (1999): Age and the role of symptomatology in readiness to quit smoking. Addict Behav 24, 1-16 
41. Colivicchi F, Mocini D, Tubaro M, Aiello A, Clavario P, Santini M (2011): Effect of smoking relapse on outcome after acute coronary syndromes. Am J Cardiol 108, 804808

42. Comstock GW, Brownlow WJ, Stone RW, Sartwell PE (1970): Cigarette smoking and changes in respiratory findings. Arch Environ Health $\underline{21}, 50-57$

43. Conlan MG, Folsom AR, Finch A, Davis CE, Sorlie P, Wu KK (1993): Correlation of plasma protein $\mathrm{C}$ levels with cardiovascular risk factors in middle-aged adults: the Atherosclerosis Risk in Communities (ARIC) Study. Thromb Haemost 70, 762-767

44. Cook DG, Shaper AG, Pocock SJ, Kussick SJ (1986): Giving up smoking and the risk of heart attacks. A report from The British Regional Heart Study. Lancet 2 , 1376-1380

45. Cornuz J, Humair JP, Seematter L, Stoianov R, van Melle G, Stalder H, Pecoud A (2002): Efficacy of resident training in smoking cessation: a randomized, controlled trial of a program based on application of behavioral theory and practice with standardized patients. Ann Intern Med 136, 429-437

46. Corrigall WA, Franklin KB, Coen KM, Clarke PB (1992): The mesolimbic dopaminergic system is implicated in the reinforcing effects of nicotine. Psychopharmacology (Berl) 107, 285-289

47. Craig WY, Palomaki GE, Haddow JE (1989): Cigarette smoking and serum lipid and lipoprotein concentrations: an analysis of published data. Bmj 298 , 784-788

48. Critchley JA, Capewell S (2003): Mortality risk reduction associated with smoking cessation in patients with coronary heart disease: a systematic review. Jama 290, 8697

49. Cryer PE, Haymond MW, Santiago JV, Shah SD (1976): Norepinephrine and epinephrine release and adrenergic mediation of smoking-associated hemodynamic and metabolic events. N Engl J Med 295, 573-577

50. Cummings SR, Coates TJ, Richard RJ, Hansen B, Zahnd EG, VanderMartin R, Duncan C, Gerbert B, Martin A, Stein MJ (1989): Training physicians in counseling about smoking cessation. A randomized trial of the "Quit for Life" program. Ann Intern Med $\underline{110}, 640-647$

51. Dani JA, Heinemann S (1996): Molecular and cellular aspects of nicotine abuse. Neuron $\underline{16}, 905-908$

52. Dani JA, De Biasi M (2001): Cellular mechanisms of nicotine addiction. Pharmacol Biochem Behav $\underline{70}$, 439-446

53. De Biasi M, Salas R (2008): Influence of neuronal nicotinic receptors over nicotine addiction and withdrawal. Exp Biol Med (Maywood) 233, 917-929 
54. DiClemente CC, Prochaska JO, Fairhurst SK, Velicer WF, Velasquez MM, Rossi JS (1991): The process of smoking cessation: an analysis of precontemplation, contemplation, and preparation stages of change. J Consult Clin Psychol 59, 295-304

55. Dietz R, Rauch B (2003): Guidelines for diagnosis and treatment of chronic coronary heart disease. Issued by the executive committee of the German Society of Cardiology--Heart Circulation Research in cooperation with the German Society for Prevention and Rehabilitation of Cardiac Diseases and the German Society for Thoracic and Cardiovascular Surgery. Z Kardiol 92, 501-521

56. Diez-Roux AV, Nieto FJ, Comstock GW, Howard G, Szklo M (1995): The relationship of active and passive smoking to carotid atherosclerosis 12-14 years later. Prev Med 24, 48-55

57. DiFranza JR, Rigotti NA, McNeill AD, Ockene JK, Savageau JA, St Cyr D, Coleman M (2000): Initial symptoms of nicotine dependence in adolescents. Tob Control $\underline{9}$, 313-319

58. Djalalinia S, Tehrani FR, Malekafzali H, Dovvom MR, Neot R, Peykari N (2011): Training of general practitioners about smoking cessation counseling. J Pak Med Assoc $\underline{61}, 449-452$

59. DKFZ (2002): Gesundheit fördern - Tabakkonsum verringern. Handlungsempfehlungen für eine wirksame Tabakkontrollpolitik in Deutschland. Rote Reihe Tabakprävention und Tabakkontrolle Heidelberg, 2002

60. Dobson AJ, Alexander HM, Heller RF, Lloyd DM (1991): How soon after quitting smoking does risk of heart attack decline? J Clin Epidemiol 44, 1247-1253

61. Doll R (1999): Risk from tobacco and potentials for health gain. Int J Tuberc Lung Dis $\underline{3}, 90-99$

62. Doll R, Hill AB (1950): Smoking and carcinoma of the lung; preliminary report. Br Med J 2, 739-748

63. Doll R, Peto R, Wheatley K, Gray R, Sutherland I (1994): Mortality in relation to smoking: 40 years' observations on male British doctors. Bmj $\underline{309}$, 901-911

64. Doll R, Peto R, Boreham J, Sutherland I (2004): Mortality in relation to smoking: 50 years' observations on male British doctors. Bmj $\underline{328}, 1519$

65. Dunner DL, Zisook S, Billow AA, Batey SR, Johnston JA, Ascher JA (1998): A prospective safety surveillance study for bupropion sustained-release in the treatment of depression. J Clin Psychiatry 59, 366-373

66. Ebbert JO, Wyatt KD, Hays JT, Klee EW, Hurt RD (2010): Varenicline for smoking cessation: efficacy, safety, and treatment recommendations. Patient Prefer Adherence $\underline{4}, 355-362$ 
67. Eliasson B, Hjalmarson A, Kruse E, Landfeldt B, Westin A (2001): Effect of smoking reduction and cessation on cardiovascular risk factors. Nicotine Tob Res $\underline{3}$, 249-255

68. Emmons KM, Goldstein MG (1992): Smokers who are hospitalized: a window of opportunity for cessation interventions. Prev Med 21, 262-269

69. Erguder IB, Erguder T, Ozkan C, Bozkurt N, Soylu K, Devrim E, Durak I (2006): Short-term effects of smoking cessation on blood antioxidant parameters and paraoxonase activity in healthy asymptomatic long-term cigarette smokers. Inhal Toxicol $\underline{18}, 575-579$

70. Fagerstrom K, Russ C, Yu CR, Yunis C, Foulds J (2012): The Fagerstrom Test for Nicotine Dependence as a predictor of smoking abstinence: a pooled analysis of varenicline clinical trial data. Nicotine Tob Res $\underline{14}$, 1467-1473

71. Ferguson JA, Patten CA, Schroeder DR, Offord KP, Eberman KM, Hurt RD (2003): Predictors of 6-month tobacco abstinence among 1224 cigarette smokers treated for nicotine dependence. Addict Behav $\underline{28}, 1203-1218$

72. Ferlay J, Shin HR, Bray F, Forman D, Mathers C, Parkin DM (2008): GLOBOCAN 2008 v2.0, Cancer Incidence and Mortality Worldwide: IARC CancerBase No. 10. Lyon, France: International Agency for Research on Cancer; 2010. Available from: http://globocan.iarc.fr, accessed on 23.11.2013.

73. Fidler JA, Wardle J, Brodersen NH, Jarvis MJ, West R (2006): Vulnerability to smoking after trying a single cigarette can lie dormant for three years or more. Tob Control $\underline{15}, 205-209$

74. Fiore M, Jaén CR, Baker TB, Bailey WC, Bennett G, Benowitz NL, Christiansen BA, Connell M, Curry SJ, Dorfman SF et al. (2008): A clinical practice guideline for treating tobacco use and dependence: 2008 update. A U.S. Public Health Service report. Am J Prev Med 35, 158-176

75. Fiore MC (2000): US public health service clinical practice guideline: treating tobacco use and dependence. Respir Care 45, 1200-1262

76. Fisher SD, Zareba W, Moss AJ, Marder VJ, Sparks CE, Hochman J, Liang C, Krone RJ (2000): Effect of smoking on lipid and thrombogenic factors two months after acute myocardial infarction. Am J Cardiol $\underline{86}$, 813-818

77. Fossati R, Apolone G, Negri E, Compagnoni A, La Vecchia C, Mangano S, Clivio L, Garattini S (2007): A double-blind, placebo-controlled, randomized trial of bupropion for smoking cessation in primary care. Arch Intern Med 167, 1791-1797

78. Freeman DJ, Griffin BA, Murray E, Lindsay GM, Gaffney D, Packard CJ, Shepherd J (1993): Smoking and plasma lipoproteins in man: effects on low density lipoprotein cholesterol levels and high density lipoprotein subfraction distribution. Eur J Clin Invest $\underline{23}, 630-640$ 
79. Freund M, Campbell E, Paul C, McElduff P, Walsh RA, Sakrouge R, Wiggers J, Knight J (2008): Smoking care provision in hospitals: a review of prevalence. Nicotine Tob Res $\underline{10}, 757-774$

80. Frishman WH, Ky T, Ismail A (2001): Tobacco smoking, nicotine, and nicotine and non-nicotine replacement therapies. Heart Dis $\underline{3}, 365-377$

81. Fryer JD, Lukas RJ (1999): Noncompetitive functional inhibition at diverse, human nicotinic acetylcholine receptor subtypes by bupropion, phencyclidine, and ibogaine. $\mathbf{J}$ Pharmacol Exp Ther 288, 88-92

82. Garrison RJ, Kannel WB, Feinleib M, Castelli WP, McNamara PM, Padgett SJ (1978): Cigarette smoking and HDL cholesterol: the Framingham offspring study. Atherosclerosis $\underline{30}, 17-25$

83. George J, Taylor S, Hong T, Leung S, Nguyen J (2012): A pilot study to investigate the scope for an inpatient smoking cessation programme. Intern Med J $\underline{42}$, e80-83

84. Gies CE, Buchman D, Robinson J, Smolen D (2008): Effect of an inpatient nursedirected smoking cessation program. West J Nurs Res $\underline{30}$, 6-19

85. Goldstein MG, Niaura R, Willey-Lessne C, DePue J, Eaton C, Rakowski W, Dube C (1997): Physicians counseling smokers. A population-based survey of patients' perceptions of health care provider-delivered smoking cessation interventions. Arch Intern Med 157, 1313-1319

86. Goldstein S, Czapski G (1995): The reaction of NO. with O2.- and HO2.: a pulse radiolysis study. Free Radic Biol Med 19, 505-510

87. Gonzales D, Rennard SI, Nides M, Oncken C, Azoulay S, Billing CB, Watsky EJ, Gong J, Williams KE, Reeves KR (2006): Varenicline, an alpha4beta2 nicotinic acetylcholine receptor partial agonist, vs sustained-release bupropion and placebo for smoking cessation: a randomized controlled trial. Jama 296, 47-55

88. Gordon T, Kannel WB, McGee D, Dawber TR (1974): Death and coronary attacks in men after giving up cigarette smoking. A report from the Framingham study. Lancet $\underline{2}$, $1345-1348$

89. Graham S, Levin ML (1971): Smoking withdrawal in the reduction of risk of lung cancer. Cancer 27, 865-871

90. Grassi G, Seravalle G, Calhoun DA, Bolla GB, Giannattasio C, Marabini M, Del Bo A, Mancia G (1994): Mechanisms responsible for sympathetic activation by cigarette smoking in humans. Circulation $\underline{90}, 248-253$

91. Grassi MC, Chiamulera C, Baraldo M, Culasso F, Ferketich AK, Raupach T, Patrono C, Nencini P (2012): Cigarette smoking knowledge and perceptions among students in four Italian medical schools. Nicotine Tob Res $\underline{14}$, 1065-1072 
92. Gross B, Brose L, Schumann A, Ulbricht S, Meyer C, Volzke H, Rumpf HJ, John U (2008): Reasons for not using smoking cessation aids. BMC Public Health $\underline{8}, 129$

93. Gryglewski RJ, Palmer RM, Moncada S (1986): Superoxide anion is involved in the breakdown of endothelium-derived vascular relaxing factor. Nature $\underline{320}, 454-456$

94. Guindon GE (2006): The cost attributable to tobacco use: a critical review of the literature. Geneva, World Health Organization, 2006

95. Gunnell D, Irvine D, Wise L, Davies C, Martin RM (2009): Varenicline and suicidal behaviour: a cohort study based on data from the General Practice Research Database. Bmj $\underline{339}$, b3805

96. Hammond EC (1966): Smoking in relation to the death rates of one million men and women. Natl Cancer Inst Monogr 19, 127-204

97. Haustein KO, Krause J, Haustein H, Rasmussen T, Cort N (2002): Effects of cigarette smoking or nicotine replacement on cardiovascular risk factors and parameters of haemorheology. J Intern Med 252, 130-139

98. Heatherton TF, Kozlowski LT, Frecker RC, Fagerstrom KO (1991): The Fagerstrom Test for Nicotine Dependence: a revision of the Fagerstrom Tolerance Questionnaire. Br J Addict 무, 1119-1127

99. Henningfield JE (1995): Nicotine medications for smoking cessation. N Engl J Med $\underline{333}, 1196-1203$

100. Henningfield JE, Keenan RM (1993): Nicotine delivery kinetics and abuse liability. J Consult Clin Psychol $\underline{61}, 743-750$

101. Herbert WH (1975): Cigarette smoking and arteriographically demonstrable coronary artery disease. Chest $\underline{67}, 49-52$

102. Hildebrand JR, Sastry S (2013): "Stop Smoking!" Do We Say It Enough? J Oncol Pract

103. Hoch E, Muehlig S, Hofler M, Lieb R, Wittchen HU (2004): How prevalent is smoking and nicotine dependence in primary care in Germany? Addiction $\underline{99}, 1586-1598$

104. Hoffmann D, Hoffmann I, El-Bayoumy K (2001): The less harmful cigarette: a controversial issue. a tribute to Ernst L. Wynder. Chem Res Toxicol 14, 767-790

105. Hosokawa S, Hiasa Y, Miyazaki S, Ogura R, Miyajima H, Ohara Y, Yuba K, Suzuki N, Takahashi T, Kishi K et al. (2008): Effects of smoking cessation on coronary endothelial function in patients with recent myocardial infarction. Int J Cardiol $\underline{128}, 48-52$

106. Howard G, Burke GL, Szklo M, Tell GS, Eckfeldt J, Evans G, Heiss G (1994): Active and passive smoking are associated with increased carotid wall thickness. The Atherosclerosis Risk in Communities Study. Arch Intern Med 154, 1277-1282 
107. Hudson NL, Mannino DM (2010): Tobacco use: a chronic illness? J Community Health $\underline{35}, 549-553$

108. Hughes JA, Hutchison DC, Bellamy D, Dowd DE, Ryan KC, Hugh-Jones P (1982): The influence of cigarette smoking and its withdrawal on the annual change of lung function in pulmonary emphysema. Q J Med $\underline{51,115-124}$

109. Hughes JR (1988): Dependence potential and abuse liability of nicotine replacement therapies. Prog Clin Biol Res 261, 261-277

110. Hughes JR, Gulliver SB, Fenwick JW, Valliere WA, Cruser K, Pepper S, Shea P, Solomon LJ, Flynn BS (1992): Smoking cessation among self-quitters. Health Psychol $\underline{11}, 331-334$

111. Hughes JR, Keely J, Naud S (2004): Shape of the relapse curve and long-term abstinence among untreated smokers. Addiction 99, 29-38

112. Hughes JR, Stead LF, Lancaster T (2007): Antidepressants for smoking cessation. Cochrane Database Syst Rev CD000031

113. IARC Working Group on the Evaluation of Carcinogenic Risks to Humans: Tobacco smoke and involuntary smoking. (IARC Monographs on the Evaluation of Carcinogenic Risks to Humans), Vol.83; IARCPress, Lyon 2004

114. Jahne A, Balmford J, Schulz C, Leifert JA (2013): Do smoking hospital inpatients get therapies for cessation? Experiences of health care professionals. Dtsch Med Wochenschr 138, 1153-1158

115. Jarvis MJ, Raw M, Russell MA, Feyerabend C (1982): Randomised controlled trial of nicotine chewing-gum. Br Med J (Clin Res Ed) 285, 537-540

116. Jha P, Ramasundarahettige C, Landsman V, Rostron B, Thun M, Anderson RN, McAfee T, Peto R (2013): 21st-century hazards of smoking and benefits of cessation in the United States. N Engl J Med $\underline{368}, 341-350$

117. Jiloha RC (2010): Biological basis of tobacco addiction: Implications for smokingcessation treatment. Indian J Psychiatry 52, 301-307

118. Jockel KH, Ahrens W, Wichmann HE, Becher H, Bolm-Audorff U, Jahn I, Molik B, Greiser E, Timm J (1992): Occupational and environmental hazards associated with lung cancer. Int J Epidemiol 21, 202-213

119. John U, Hanke M (2001): Tobacco smoking attributable mortality in Germany. Gesundheitswesen $\underline{63}, 363-369$

120. Jorenby DE, Leischow SJ, Nides MA, Rennard SI, Johnston JA, Hughes AR, Smith SS, Muramoto ML, Daughton DM, Doan K et al. (1999): A controlled trial of sustained-release bupropion, a nicotine patch, or both for smoking cessation. N Engl $\mathbf{J}$ Med $\underline{340}$, 685-691 
121. Jorenby DE, Hays JT, Rigotti NA, Azoulay S, Watsky EJ, Williams KE, Billing CB, Gong J, Reeves KR (2006): Efficacy of varenicline, an alpha4beta2 nicotinic acetylcholine receptor partial agonist, vs placebo or sustained-release bupropion for smoking cessation: a randomized controlled trial. Jama $\underline{296}$, 56-63

122. Joseph AM, Norman SM, Ferry LH, Prochazka AV, Westman EC, Steele BG, Sherman SE, Cleveland M, Antonuccio DO, Hartman N et al. (1996): The safety of transdermal nicotine as an aid to smoking cessation in patients with cardiac disease. N Engl J Med $\underline{335}, 1792-1798$

123. Karatzi K, Papamichael C, Karatzis E, Papaioannou TG, Stamatelopoulos K, Zakopoulos NA, Zampelas A, Lekakis J (2007): Acute smoke-induced endothelial dysfunction is more prolonged in smokers than in non-smokers. Int J Cardiol $\underline{120}$, 404-406

124. Katz DA, Tang F, Faseru B, Horwitz PA, Jones P, Spertus J (2011): Prevalence and correlates of smoking cessation pharmacotherapy in hospitalized smokers with acute myocardial infarction. Am Heart J $\underline{162}, 74-80$

125. Katz DA, Vander Weg MW, Holman J, Nugent A, Baker L, Johnson S, Hillis SL, Titler M (2012): The Emergency Department Action in Smoking Cessation (EDASC) trial: impact on delivery of smoking cessation counseling. Acad Emerg Med 19, 409420

126. Kerstjens HA, Rijcken B, Schouten JP, Postma DS (1997): Decline of FEV1 by age and smoking status: facts, figures, and fallacies. Thorax $\underline{52}, 820-827$

127. King N: Using Templates in the thematic analysis of text; in: Essential Guide to qualitative methods in organizational research; hrsg. v. Cassell C, Symon G; Sage Publications, London 2004, 256-270

128. Kojda G, Harrison D (1999): Interactions between NO and reactive oxygen species: pathophysiological importance in atherosclerosis, hypertension, diabetes and heart failure. Cardiovasc Res $\underline{43}, 562-571$

129. Kraus L, Augustin R (2001): Repräsentativerhebung zum Gebrauch psychoaktiver Substanzen bei Erwachsenen in Deutschland 2000. Sucht 47, 3-86

130. Krupski WC (1991): The peripheral vascular consequences of smoking. Ann Vasc Surg $\underline{5}, 291-304$

131. Lampert T (2011): Rauchen - Aktuelle Entwicklungen bei Erwachsenen. Hrsg Robert Koch-Institut Berlin GBE kompakt 2(4)

132. Lancaster T, Stead LF (2005a): Individual behavioural counselling for smoking cessation. Cochrane Database Syst Rev CD001292

133. Lancaster T, Stead LF (2005b): Self-help interventions for smoking cessation. Cochrane Database Syst Rev CD001118 
134. Lau J, Antman EM, Jimenez-Silva J, Kupelnick B, Mosteller F, Chalmers TC (1992): Cumulative meta-analysis of therapeutic trials for myocardial infarction. $\mathrm{N}$ Engl $\mathbf{J}$ Med $\underline{327}, 248-254$

135. Lokke A, Lange P, Scharling H, Fabricius P, Vestbo J (2006): Developing COPD: a 25 year follow up study of the general population. Thorax $\underline{61}, 935-939$

136. Louis M, Clarke PB (1998): Effect of ventral tegmental 6-hydroxydopamine lesions on the locomotor stimulant action of nicotine in rats. Neuropharmacology $\underline{37}, 1503-$ 1513

137. Lyness WH, Friedle NM, Moore KE (1979): Destruction of dopaminergic nerve terminals in nucleus accumbens: effect on d-amphetamine self-administration. Pharmacol Biochem Behav 11, 553-556

138. Mansvelder HD, McGehee DS (2002): Cellular and synaptic mechanisms of nicotine addiction. J Neurobiol 53, 606-617

139. Mansvelder HD, Keath JR, McGehee DS (2002): Synaptic mechanisms underlie nicotine-induced excitability of brain reward areas. Neuron $\underline{33}, 905-919$

140. Mansvelder HD, De Rover M, McGehee DS, Brussaard AB (2003): Cholinergic modulation of dopaminergic reward areas: upstream and downstream targets of nicotine addiction. Eur J Pharmacol $\underline{480}, 117-123$

141. Mansvelder HD, Fagen ZM, Chang B, Mitchum R, McGehee DS (2007): Bupropion inhibits the cellular effects of nicotine in the ventral tegmental area. Biochem Pharmacol 74, 1283-1291

142. Mao Y, Hu J, Ugnat AM, Semenciw R, Fincham S (2001): Socioeconomic status and lung cancer risk in Canada. Int J Epidemiol 30, 809-817

143. Mathers CD, Loncar D (2006): Projections of global mortality and burden of disease from 2002 to 2030. PLoS Med $\underline{3}$, e442

144. Mausner JS, Mausner B, Rial WY (1968): The influence of a physician on the smoking of his patients. Am J Public Health Nations Health $\underline{58}$, 46-53

145. McBride CM, Emmons KM, Lipkus IM (2003): Understanding the potential of teachable moments: the case of smoking cessation. Health Educ Res $\underline{18}, 156-170$

146. McCarty MC, Zander KM, Hennrikus DJ, Lando HA (2001): Barriers among nurses to providing smoking cessation advice to hospitalized smokers. Am J Health Promot $\underline{16}$, $85-87$, ii

147. McEwen A, West R (2001): Smoking cessation activities by general practitioners and practice nurses. Tob Control $\underline{10}, 27-32$

148. McGill HC, Jr. (1988): The cardiovascular pathology of smoking. Am Heart J $\underline{115}$, 250-257 
149. Meine TJ, Patel MR, Washam JB, Pappas PA, Jollis JG (2005): Safety and effectiveness of transdermal nicotine patch in smokers admitted with acute coronary syndromes. Am J Cardiol 95, 976-978

150. Meyer C, Rumpf HJ, Hapke U, John U (2000): Utilisation of Aids for Smoking Cessation. Sucht $2000 \underline{46}, 398-407$

151. Michie S, van Stralen MM, West R (2011): The behaviour change wheel: a new method for characterising and designing behaviour change interventions. Implement Sci $\underline{6}$, 42

152. Mills CA, Porter MM (1950): Tobacco smoking habits and cancer of the mouth and respiratory system. Cancer Res $\underline{10}, 539-542$

153. Mohiuddin SM, Mooss AN, Hunter CB, Grollmes TL, Cloutier DA, Hilleman DE (2007): Intensive smoking cessation intervention reduces mortality in high-risk smokers with cardiovascular disease. Chest $\underline{131}, 446-452$

154. Morgan MD, Britton JR (2003): Chronic obstructive pulmonary disease 8: nonpharmacological management of COPD. Thorax $\underline{58}, 453-457$

155. Muhlig S, Haarig F, Teumer G, Bothen E, Nehrkorn M, Berndt C, Rustler C (2013): German Network for Tobacco-Free Hospitals \& Health-Care Services. Gesundheitswesen

156. Munjal S, Koval T, Muhammad R, Jin Y, Demmel V, Roethig HJ, Mendes P, Unverdorben M (2009): Heart rate variability increases with reductions in cigarette smoke exposure after 3 days. J Cardiovasc Pharmacol Ther 14, 192-198

157. Murray CJ, Lopez AD (1997): Alternative projections of mortality and disability by cause 1990-2020: Global Burden of Disease Study. Lancet 349, 1498-1504

158. Murray RL, Leonardi-Bee J, Marsh J, Jayes L, Li J, Parrott S, Britton J (2013): Systematic identification and treatment of smokers by hospital based cessation practitioners in a secondary care setting: cluster randomised controlled trial. Bmj $\underline{347}, \mathrm{f} 4004$

159. Nedeljkovic ZS, Gokce N, Loscalzo J (2003): Mechanisms of oxidative stress and vascular dysfunction. Postgrad Med J $\underline{79}$, 195-199; quiz 198-200

160. Negri E, La Vecchia C, D'Avanzo B, Nobili A, La Malfa RG (1994): Acute myocardial infarction: association with time since stopping smoking in Italy. GISSI-EFRIM Investigators. Gruppo Italiano per lo Studio della Sopravvivenza nell'Infarto. Epidemiologia dei Fattori di Rischio dell'Infarto Miocardico. J Epidemiol Community Health $\underline{48}, 129-133$

161. Nestler EJ (2005): Is there a common molecular pathway for addiction? Nat Neurosci $\underline{8}, 1445-1449$ 
162. Neubauer S, Welte R, Beiche A, Koenig HH, Buesch K, Leidl R (2006): Mortality, morbidity and costs attributable to smoking in Germany: update and a 10-year comparison. Tob Control $\underline{15}, 464-471$

163. OECD (2012): Health at a Glance: Europe 2012. OECD Publishing

164. Oren S, Isakov I, Golzman B, Kogan J, Turkot S, Peled R, Yosefy C (2006): The influence of smoking cessation on hemodynamics and arterial compliance. Angiology $\underline{57}, 564-568$

165. Osann KE, Lowery JT, Schell MJ (2000): Small cell lung cancer in women: risk associated with smoking, prior respiratory disease, and occupation. Lung Cancer $\underline{28}, 1-10$

166. Pauwels RA, Rabe KF (2004): Burden and clinical features of chronic obstructive pulmonary disease (COPD). Lancet $\underline{364}$, 613-620

167. Peat JK, Woolcock AJ, Cullen K (1990): Decline of lung function and development of chronic airflow limitation: a longitudinal study of non-smokers and smokers in Busselton, Western Australia. Thorax 45, 32-37

168. Perk J, De Backer G, Gohlke H, Graham I, Reiner Z, Verschuren M, Albus C, Benlian P, Boysen G, Cifkova R et al. (2012): European Guidelines on cardiovascular disease prevention in clinical practice (version 2012). The Fifth Joint Task Force of the European Society of Cardiology and Other Societies on Cardiovascular Disease Prevention in Clinical Practice (constituted by representatives of nine societies and by invited experts). Eur Heart J $\underline{33}$, 1635-1701

169. Peto R, Darby S, Deo H, Silcocks P, Whitley E, Doll R (2000): Smoking, smoking cessation, and lung cancer in the UK since 1950: combination of national statistics with two case-control studies. Bmj $\underline{321}$, 323-329

170. Pidoplichko VI, DeBiasi M, Williams JT, Dani JA (1997): Nicotine activates and desensitizes midbrain dopamine neurons. Nature $\underline{390}$, 401-404

171. Pipe AL, Eisenberg MJ, Gupta A, Reid RD, Suskin NG, Stone JA (2011): Smoking cessation and the cardiovascular specialist: Canadian Cardiovascular Society position paper. Can J Cardiol 27, 132-137

172. Pirie K, Peto R, Reeves GK, Green J, Beral V (2013): The 21st century hazards of smoking and benefits of stopping: a prospective study of one million women in the UK. Lancet $\underline{381}, 133-141$

173. Postma DS, de Vries K, Koeter GH, Sluiter HJ (1986): Independent influence of reversibility of air-flow obstruction and nonspecific hyperreactivity on the long-term course of lung function in chronic air-flow obstruction. Am Rev Respir Dis 134, 276280

174. Prescott E, Bjerg AM, Andersen PK, Lange P, Vestbo J (1997): Gender difference in smoking effects on lung function and risk of hospitalization for COPD: results from a Danish longitudinal population study. Eur Respir J $\underline{10}$, 822-827 
175. Prochaska JJ, Hilton JF (2012): Risk of cardiovascular serious adverse events associated with varenicline use for tobacco cessation: systematic review and meta-analysis. Bmj $\underline{344}$, e2856

176. Prochaska JO, Velicer WF (1997): The transtheoretical model of health behavior change. Am J Health Promot $\underline{12}, 38-48$

177. Quillen JE, Rossen JD, Oskarsson HJ, Minor RL, Jr., Lopez AG, Winniford MD (1993): Acute effect of cigarette smoking on the coronary circulation: constriction of epicardial and resistance vessels. J Am Coll Cardiol 22, 642-647

178. Radi R, Beckman JS, Bush KM, Freeman BA (1991): Peroxynitrite-induced membrane lipid peroxidation: the cytotoxic potential of superoxide and nitric oxide. Arch Biochem Biophys $\underline{288}, 481-487$

179. Ramsdale DR, Faragher EB, Bray CL, Bennett DH, Ward C, Beton DC (1985): Smoking and coronary artery disease assessed by routine coronary arteriography. Br Med $\mathrm{J}$ (Clin Res Ed) 290, 197-200

180. Raupach T, Shahab L, Eimer S, Puls M, Hasenfuss G, Andreas S (2010): Increasing the use of nicotine replacement therapy by a simple intervention: an exploratory trial. Subst Use Misuse $\underline{45}$, 403-413

181. Raupach T, Merker J, Hasenfuss G, Andreas S, Pipe A (2011): Knowledge gaps about smoking cessation in hospitalized patients and their doctors. Eur J Cardiovasc Prev Rehabil 18, 334-341

182. Raupach T, Strobel L, Beard E, Krampe H, Anders S, West R (2013): German medical students' beliefs about the effectiveness of different methods of stopping smoking. Nicotine Tob Res $\underline{15}$, 1892-1901

183. Rea TD, Heckbert SR, Kaplan RC, Smith NL, Lemaitre RN, Psaty BM (2002): Smoking status and risk for recurrent coronary events after myocardial infarction. Ann Intern Med 137, 494-500

184. Reid RD, Pipe AL, Quinlan B (2006): Promoting smoking cessation during hospitalization for coronary artery disease. Can J Cardiol 22, 775-780

185. Reid RD, Mullen KA, Slovinec D'Angelo ME, Aitken DA, Papadakis S, Haley PM, McLaughlin CA, Pipe AL (2010): Smoking cessation for hospitalized smokers: an evaluation of the "Ottawa Model". Nicotine Tob Res $\underline{12}$, 11-18

186. Reinders JH, Brinkman HJ, van Mourik JA, de Groot PG (1986): Cigarette smoke impairs endothelial cell prostacyclin production. Arteriosclerosis $\underline{6}, 15-23$

187. Rennard S, Hughes J, Cinciripini PM, Kralikova E, Raupach T, Arteaga C, St Aubin LB, Russ C (2012): A randomized placebo-controlled trial of varenicline for smoking cessation allowing flexible quit dates. Nicotine Tob Res $\underline{14}, 343-350$ 
188. Rice VH, Hartmann-Boyce J, Stead LF (2013): Nursing interventions for smoking cessation. Cochrane Database Syst Rev $\underline{8}$, CD001188

189. Rigotti NA, Munafo MR, Stead LF (2007): Interventions for smoking cessation in hospitalised patients. Cochrane Database Syst Rev CD001837

190. Rigotti NA, Munafo MR, Stead LF (2008): Smoking cessation interventions for hospitalized smokers: a systematic review. Arch Intern Med 168, 1950-1960

191. Rigotti NA, Clair C, Munafo MR, Stead LF (2012): Interventions for smoking cessation in hospitalised patients. Cochrane Database Syst Rev $\underline{5}$, CD001837

192. Risch HA, Howe GR, Jain M, Burch JD, Holowaty EJ, Miller AB (1993): Are female smokers at higher risk for lung cancer than male smokers? A case-control analysis by histologic type. Am J Epidemiol 138, 281-293

193. RKI - Robert-Koch Institut (2010): Verbreitung von Krebserkrankungen in Deutschland. Entwicklung der Prävalenzen zwischen 1990 und 2010. Beiträge zur Gesundheitsberichterstattung des Bundes. RKI, Berlin. 2010

194. Roberts DL (1988): Natural tobacco flavor. Recent Adv Tob Sci $\underline{14}$, 49-81

195. Roddy E, Rubin P, Britton J (2004): A study of smoking and smoking cessation on the curricula of UK medical schools. Tob Control $\underline{13}, 74-77$

196. Rollema H, Coe JW, Chambers LK, Hurst RS, Stahl SM, Williams KE (2007): Rationale, pharmacology and clinical efficacy of partial agonists of alpha4beta2 nACh receptors for smoking cessation. Trends Pharmacol Sci $\underline{28}, 316-325$

197. Rosenberg L, Kaufman DW, Helmrich SP, Shapiro S (1985): The risk of myocardial infarction after quitting smoking in men under 55 years of age. N Engl J Med $\underline{313}$, $1511-1514$

198. Rosenberg L, Palmer JR, Shapiro S (1990): Decline in the risk of myocardial infarction among women who stop smoking. N Engl J Med 222, 213-217

199. Ruge J, Ulbricht S, Schumann A, Rumpf HJ, John U, Meyer C (2008): Intention to quit smoking: is the partner's smoking status associated with the smoker's intention to quit? Int J Behav Med 15, 328-335

200. Rupp A, Neudert M, Hetzel M (2010): Smoking cessation programmes at hospitals with departments for lung diseases. Dtsch Med Wochenschr 135, 501-506

201. Russell MA (1980): Nicotine intake and its regulation. J Psychosom Res $\underline{24}$, 253-264

202. Rylander R, Axelsson G, Andersson L, Liljequist T, Bergman B (1996): Lung cancer, smoking and diet among Swedish men. Lung Cancer 14 Suppl 1, S75-83 
203. Sajdak R, Trembath L, Thomas KS (2013): The Importance of Standard Operating Procedures in Clinical Trials. J Nucl Med Technol

204. Sankaranarayanan R, Varghese C, Duffy SW, Padmakumary G, Day NE, Nair MK (1994): A case-control study of diet and lung cancer in Kerala, south India. Int J Cancer $\underline{58}, 644-649$

205. Scanlon PD, Connett JE, Waller LA, Altose MD, Bailey WC, Buist AS (2000): Smoking cessation and lung function in mild-to-moderate chronic obstructive pulmonary disease. The Lung Health Study. Am J Respir Crit Care Med 161, 381-390

206. Schmid I (2012): How to develop a standard operating procedure for sorting unfixed cells. Methods 57, 392-397

207. Schmidt LG, Gastpar M, Falkai P, Gäbel W (2006): Evidenzbasierte Suchtmedizin. Behandlungsleitlinie Substanzbezogene Störungen. Deutscher Ärzte Verlag

208. Schrek R, Baker LA (1950): Tobacco smoking as an etiologic factor in disease; cancer. Cancer Res $\underline{10}, 49-58$

209. Schultz AS, Finegan B, Nykiforuk CI, Kvern MA (2011): A qualitative investigation of smoke-free policies on hospital property. Cmaj $\underline{183}$, E1334-1344

210. Sees KL (1990): Cigarette smoking, nicotine dependence, and treatment. West J Med $\underline{152}, 578-584$

211. Shahab L, Jarvis MJ, Britton J, West R (2006): Prevalence, diagnosis and relation to tobacco dependence of chronic obstructive pulmonary disease in a nationally representative population sample. Thorax $\underline{61}, 1043-1047$

212. Shimada S, Hasegawa K, Wada H, Terashima S, Satoh-Asahara N, Yamakage H, Kitaoka S, Akao M, Shimatsu A, Takahashi Y (2010): High blood viscosity is closely associated with cigarette smoking and markedly reduced by smoking cessation. Circ $\mathbf{J}$ $\underline{75}, 185-189$

213. Siafakas NM, Vermeire P, Pride NB, Paoletti P, Gibson J, Howard P, Yernault JC, Decramer M, Higenbottam T, Postma DS et al. (1995): Optimal assessment and management of chronic obstructive pulmonary disease (COPD). The European Respiratory Society Task Force. Eur Respir J $\underline{8}, 1398-1420$

214. Silagy C, Lancaster T, Stead L, Mant D, Fowler G (2004): Nicotine replacement therapy for smoking cessation. Cochrane Database Syst Rev CD000146

215. Simonato L, Agudo A, Ahrens W, Benhamou E, Benhamou S, Boffetta P, Brennan P, Darby SC, Forastiere F, Fortes C et al. (2001): Lung cancer and cigarette smoking in Europe: an update of risk estimates and an assessment of inter-country heterogeneity. Int J Cancer 91, 876-887 
216. Sinha S, Luben RN, Welch A, Bingham S, Wareham NJ, Day NE, Khaw KT (2005): Fibrinogen and cigarette smoking in men and women in the European Prospective Investigation into Cancer in Norfolk (EPIC-Norfolk) population. Eur J Cardiovasc Prev Rehabil $\underline{12}, 144-150$

217. Springer CM, Tannert Niang KM, Matte TD, Miller N, Bassett MT, Frieden TR (2008): Do medical students know enough about smoking to help their future patients? Assessment of New York City fourth-year medical students' knowledge of tobacco cessation and treatment for nicotine addiction. Acad Med $\underline{83}, 982-989$

218. Statistisches Bundesamt (2010): Mikrozensus - Fragen zur Gesundheit. Zweigstelle Bonn.

219. Statistisches Bundesamt: Grunddaten der Krankenhäuser - Fachserie 12 Reihe 6.1.1 2012. Statistisches Bundesamt, Wiesbaden 2013

220. Stead LF, Lancaster T (2005): Group behaviour therapy programmes for smoking cessation. Cochrane Database Syst Rev CD001007

221. Stead LF, Bergson G, Lancaster T (2008): Physician advice for smoking cessation. Cochrane Database Syst Rev CD000165

222. Stevens VJ, Glasgow RE, Hollis JF, Lichtenstein E, Vogt TM (1993): A smokingcessation intervention for hospital patients. Med Care $\underline{31}$, 65-72

223. Stolerman IP, Jarvis MJ (1995): The scientific case that nicotine is addictive. Psychopharmacology (Berl) 117, 2-10; discussion 14-20

224. Strobel L, Schneider NK, Krampe H, Beissbarth T, Pukrop T, Anders S, West R, Aveyard P, Raupach T (2012): German medical students lack knowledge of how to treat smoking and problem drinking. Addiction 107, 1878-1882

225. Teo KK, Ounpuu S, Hawken S, Pandey MR, Valentin V, Hunt D, Diaz R, Rashed W, Freeman R, Jiang L et al. (2006): Tobacco use and risk of myocardial infarction in 52 countries in the INTERHEART study: a case-control study. Lancet $\underline{368}, 647-658$

226. Thomson NC, Spears M (2005): The influence of smoking on the treatment response in patients with asthma. Curr Opin Allergy Clin Immunol $\underline{5}$, 57-63

227. Thyrian JR, Panagiotakos DB, Polychronopoulos E, West R, Zatonski W, John U (2008): The relationship between smokers' motivation to quit and intensity of tobacco control at the population level: a comparison of five European countries. BMC Public Health $\underline{8}, 2$

228. Tonnesen P, Fryd V, Hansen M, Helsted J, Gunnersen AB, Forchammer H, Stockner M (1988): Effect of nicotine chewing gum in combination with group counseling on the cessation of smoking. N Engl J Med $\underline{318}, 15-18$ 
229. Tonnesen P, Tonstad S, Hjalmarson A, Lebargy F, Van Spiegel PI, Hider A, Sweet R, Townsend J (2003): A multicentre, randomized, double-blind, placebo-controlled, 1year study of bupropion SR for smoking cessation. J Intern Med 254, 184-192

230. Transdermal Nicotine Study Group (1991): Transdermal nicotine for smoking cessation. Six-month results from two multicenter controlled clinical trials. Transdermal Nicotine Study Group. Jama 266, 3133-3138

231. Trap-Jensen J (1988): Effects of smoking on the heart and peripheral circulation. Am Heart J $\underline{115}$, 263-267

232. Tuomilehto J, Tanskanen A, Salonen JT, Nissinen A, Koskela K (1986): Effects of smoking and stopping smoking on serum high-density lipoprotein cholesterol levels in a representative population sample. Prev Med 15, 35-45

233. Tzivoni D, Keren A, Meyler S, Khoury Z, Lerer T, Brunel P (1998): Cardiovascular safety of transdermal nicotine patches in patients with coronary artery disease who try to quit smoking. Cardiovasc Drugs Ther $\underline{12}, 239-244$

234. US DHHS - US Department of Health and Human Services (2004): The Health Consequences of Smoking: A Report of the Surgeon General. Atlanta, GA: U.S. Department of Health and Human Services, Centers for Disease Control and Prevention, National Center for Chronic Disease Prevention and Health Promotion, Office on Smoking and Health, 2004

235. Vega S, Stolare I (2010): Smoking cessation education increases interventions in a New Zealand hospital: World No Tobacco Day revisited. N Z Med J 123, 35-40

236. Walters N, Coleman T (2002): Comparison of the smoking behaviour and attitudes of smokers who attribute respiratory symptoms to smoking with those who do not. $\mathrm{Br} \mathbf{J}$ Gen Pract 52, 132-134

237. Wannamethee SG, Lowe GD, Shaper AG, Rumley A, Lennon L, Whincup PH (2005): Associations between cigarette smoking, pipe/cigar smoking, and smoking cessation, and haemostatic and inflammatory markers for cardiovascular disease. Eur Heart J $\underline{26}$, $1765-1773$

238. West R, McNeill A, Raw M (2000): Smoking cessation guidelines for health professionals: an update. Health Education Authority. Thorax 55, 987-999

239. White CR, Brock TA, Chang LY, Crapo J, Briscoe P, Ku D, Bradley WA, Gianturco SH, Gore J, Freeman BA et al. (1994): Superoxide and peroxynitrite in atherosclerosis. Proc Natl Acad Sci U S A $\underline{91}$, 1044-1048

240. World Health Organization: The global burden of disease: 2004 update. World Health Organization, Geneva 2008a

241. World Health Organization: WHO Report on the Global Tobacco Epidemic, 2008: The MPOWER package. World Health Organization, Geneva 2008b 
242. Wigle DT, Mao Y, Grace M (1980): Relative importance of smoking as a risk factor for selected cancers. Can J Public Health $\underline{71}$, 269-275

243. Willemse BW, Postma DS, Timens W, ten Hacken NH (2004): The impact of smoking cessation on respiratory symptoms, lung function, airway hyperresponsiveness and inflammation. Eur Respir J 23, $464-476$

244. Williams KE, Reeves KR, Billing CB, Jr., Pennington AM, Gong J (2007): A doubleblind study evaluating the long-term safety of varenicline for smoking cessation. Curr Med Res Opin 23, 793-801

245. Williams P, Robinson D, Bailey A (1979): High-density lipoprotein and coronary risk factors in normal men. Lancet $\underline{1}, 72-75$

246. Wilson K, Gibson N, Willan A, Cook D (2000): Effect of smoking cessation on mortality after myocardial infarction: meta-analysis of cohort studies. Arch Intern Med $\underline{160}, 939-944$

247. Winniford MD (1990): Smoking and cardiovascular function. J Hypertens Suppl $\underline{8}$, S17-23

248. Woolf KJ, Zabad MN, Post JM, McNitt S, Williams GC, Bisognano JD (2012): Effect of nicotine replacement therapy on cardiovascular outcomes after acute coronary syndromes. Am J Cardiol 110, 968-970

249. Wooltorton JR, Pidoplichko VI, Broide RS, Dani JA (2003): Differential desensitization and distribution of nicotinic acetylcholine receptor subtypes in midbrain dopamine areas. J Neurosci $\underline{23}, 3176-3185$

250. Wynder EL, Graham EA (1950): Tobacco smoking as a possible etiologic factor in bronchiogenic carcinoma; a study of 684 proved cases. J Am Med Assoc 143, 329-336

251. Yanbaeva DG, Dentener MA, Creutzberg EC, Wesseling G, Wouters EF (2007): Systemic effects of smoking. Chest $\underline{131}, 1557-1566$

252. Yarnell JW, Sweetnam PM, Rogers S, Elwood PC, Bainton D, Baker IA, Eastham R, O'Brien JR, Etherington MD (1987): Some long term effects of smoking on the haemostatic system: a report from the Caerphilly and Speedwell Collaborative Surveys. J Clin Pathol 40, 909-913

253. Zamir Z, Mahmud A, Feely J (2006): Acute haemodynamic effects of cigarette smoking in healthy young subjects. Ir J Med Sci 175, 20-23

254. Zeiher AM, Schachinger V, Minners J (1995): Long-term cigarette smoking impairs endothelium-dependent coronary arterial vasodilator function. Circulation $\underline{92}$, 10941100 


\section{Danksagung}

Der größte Dank gilt meinem Doktorvater Herrn PD Dr. Tobias Raupach für die herausragende und intensive Betreuung dieser Arbeit.

Als besonders anerkennenswert sind die unkomplizierte und zu jeder Zeit mögliche Rücksprache einschließlich der umgehenden Klärung problematischer Sachverhalte, die überaus konstruktive inhaltliche Kritik sowie die zahlreichen Denkanstöße hervorzuheben. Sein großes Engagement war darüber hinaus ein zusätzlicher motivierender Faktor.

Hierfür ebenso wie für die angenehme und tolle Zusammenarbeit möchte ich mich ganz herzlich bedanken.

Weiterhin danke ich allen unmittelbar an dem Projekt Beteiligten. Im Einzelnen sind dies Herr Peter Lindinger und Frau Anna Ludwig für ihre Mitwirkung an der Mitarbeiterschulung, Frau Christa Rustler vom DNRfK für die konstruktiven Vorschläge bei der Entwicklung der Fragebogen, bei der ebenfalls Herr Lindinger involviert war, sowie insbesondere Frau Sarah Schiekirka für die Durchführung der Einzelinterviews.

Ferner möchte ich mich bei der Ebenenleitung der betreffenden Studienstationen für ihre Unterstützung bedanken ebenso wie bei den Mitarbeitern des Ebenenbüros für die administrative Arbeit in Bezug auf die Probandenrekrutierung.

Mein herzlicher Dank gilt selbstverständlich auch allen teilnehmenden ärztlichen und pflegerischen Mitarbeitern sowie den Patienten für die damit verbundenden interessanten und aufschlussreichen Gespräche.

Abschließend bedanke ich mich bei Lea Kaminski und Gerrit Falk für den motivierenden Zuspruch und die Hilfe bei der Durchsicht der Arbeit. 